\title{
A Total Factor Productivity Toolbox for MATLAB
}

\author{
Bert M. Balk \\ Rotterdam School of Management, Erasmus University \\ Javier Barbero \\ European Commission, Joint Research Centre \\ José L. Zofío \\ Universidad Autónoma de Madrid \\ Erasmus Research Institute of Management, Erasmus University \\ Draft, May 15, 2018
}

\begin{abstract}
Total Factor Productivity Toolbox is a new package for MATLAB that includes functions to calculate the main Total Factor Productivity (TFP) indices and their decompositions, based on Shephard's distance functions and using Data Envelopment Analysis (DEA) programming techniques. The package includes code for the standard Malmquist, Moorsteen-Bjurek, price-weighted and share-weighted TFP indices, allowing for the choice of orientation (input or output), reference period (base, comparison, geometric mean), returns to scale (variable or constant), and specific decompositions (aggregate or identifying scale effects as well as input and output mix effects). Classic definitions of TFP corresponding to the Laspeyres, Paasche, Fisher, or Törnqvist formulas can also be calculated as particular cases. This paper describes the methodology and implementation of the functions and reports numerical results so as to ease the comparison between indices and illustrate their use.
\end{abstract}

Keywords: Total factor productivity, Malmquist, Moorsteen-Bjurek, Fisher, Törnqvist, distance function, data envelopment analysis, MATLAB.

\section{Introduction}

Total factor productivity (TFP) change is an important concept in economics because it measures the ability of firms, industries, and national economies to increase the aggregate volume of outputs they yield, relative to the aggregate volume of inputs they use. TFP constitutes a standard instrument for monitoring and benchmarking observations, and represents the cornerstone in multilateral studies of technological and economic performance, OECD (2001). Defined as the ratio of an output quantity index to an input quantity index, TFP change can be calculated and decomposed in various ways. 
TFP measurement has grown in importance over the past decades due to the increasing availability of data to study the productive performance of units, regardless of their market, governmental, or not-for-profit orientation. However, there are many ways to measure TFP depending on whether quantities and prices are available. TFP measurement requires the aggregation of quantities through suitable functions. If prices are available, it is possible to aggregate output and input quantities by index formulas of Laspeyres, Paasche, Fisher, or Törnqvist. If only quantities are available, the concept of distance function as introduced by Shephard emerges as the building block in the definition of Malmquist and MoorsteenBjurek indices. Aggregation then relies on optimization - mathematical programming techniques such as the non-parametric Data Envelopment Analysis (DEA, Cooper, Seiford, and Tone (2007)).

There are also alternative ways to decompose TFP change to identify the components. This applies both to the classical definitions using prices as aggregators, and those relying only on quantities through distance functions. These components are (technical) efficiency change, technological change, scale effect, and changes in the mix of inputs and outputs. The alternative definitions of these terms that have been proposed over the years gave rise to a healthy debate between authors, including Färe, Grosskopf, Norris, and Zhang (1994), Ray and Desli (1997), Balk (2001), Balk (2003), Lovell (2003), Zofío (2007), and Färe, Grosskopf, and Margaritis (2008). Balk and Zofío (2018) examine how to identify those terms that allow a meaningful interpretation and decomposition of TFP in a general framework. Although this paper is self-contained, the toolbox follows these authors, where more detailed theoretical and empirical considerations can be found. The toolbox implements several functions to calculate the main quantity-only and price-based TFP indices proposed in the literature, along with their associated decompositions. The toolbox allows for a choice of orientation (input or output), reference period (base, comparison, geometric mean), returns to scale (variable or constant), and specific decompositions (aggregate or identifying scale effects, as well as input and output mix effects). The code calculates Shephard's input and output distance functions approximating a production technology empirically through DEA. The toolbox also calculates classic TFP measures that do not rely on distance functions for their definition, including Laspeyres, Paasche, Fisher, and Törnqvist.

Quantity-only TFP indices based on distance functions, corresponding mainly to the Malmquist index, and coupled occasionally with the Moorsteen-Bjurek index, can be found in standard software packages like Stata (StataCorp (2015)); through user-written commands as Lee, Lee, and Lim (2011); in LIMDEP (Econometric Software (2014)); is available in dedicated noncommercial software accompanying academic handbooks such as in Cooper et al. (2007) and Bogetoft and Otto (2011) (implemented in R); in commercial software, including trial versions (O'Donnell (2011) and Emrouznejad and Thanassoulis (2011)); in free-ware programs (Coelli (1998)); and even in tutorials for spreadsheets (Zhu (2014)). For the classic TFP indices using prices as aggregators, Shehata and Mickaiel (2015) developed a Stata module to calculate value index numbers, while Coelli (1999) offered a stand-alone program that calculates Fisher and Törnqvist indices, including transitive versions using the EKS method. O'Donnell (2011) expanded this last option to Lowe-type indices under a commercial license. A recent and comprehensive review of the available general purpose and dedicated software options for efficiency and productivity analysis can be found in Daraio, Kerstens, Nepomuceno, and Sickles (2017).

Although these packages implement the main TFP indices, there is a lack of a full set of 
functions for MATLAB (The MathWorks, Inc. 2017), and none of them includes a complete decomposition of productivity change according to its multiple components. Thus, besides implementing the basic TFP definitions based on quantities and prices in the MATLAB environment, our toolbox calculates a large array of index numbers capturing (technical) efficiency change, technological change, as well as scale effects, and input and output mix effects. Quantities-only Malmquist and Moorsteen-Bjurek indices, as well as price-based Fisher and Törnqvist indices, are decomposed into mutually exclusive factors with meaningful interpretations in terms of economic index number theory. The Total Factor Productivity Toolbox introduces a set of functions, covering a wide range of TFP indices, and reports numerical results using a common example to ease comparability and to illustrate their use. The toolbox is available as free software, under the GNU General Public License version 3, and can be downloaded from http://www.tfptoolbox.com, with all the supplementary material (data, examples, and source code) to replicate all the results presented in this paper. The toolbox is also hosted on an open source repository on GitHub. ${ }^{1}$

This paper is organized as follows. Section 2 presents the data structures characterizing the production possibility sets, the structure of the functions, results, etc.. Section 3 covers the Malmquist productivity indices introduced by Caves, Christensen, and Diewert (1982) and operationalized by Färe et al. (1994), relying on radial output or input distance functions. We show how these indices can be decomposed into factors with meaningful interpretations, such as technical efficiency change, technological change, scale effect, and input and output mix effect. We also relate and interpret these factors in terms of the output code that is obtained when running the specific functions. Malmquist productivity indices take into consideration only one of the two sides of the production process, output or input. In Section 4 we consider the class of Moorsteen-Bjurek indices, defined as ratio of an output quantity index to an input quantity index, which in turn can be expressed in terms of output and input distance functions, respectively. We present the decomposition of these indices using various alternatives to identify the above effects. If input and output prices are available, it is possible to calculate and decompose classical indices such as Fisher and Törnqvist. The price-weighted productivity indices are considered in Section 5. Rather than multiplying input and output quantities by their prices, one can aggregate individual quantity ratios by a geometric mean, weighing with cost or revenue shares. Section 6 deals with the class of share-weighted indices, whose best known representative is the Törnqvist productivity index. Advanced options, including displaying and exporting results are discussed in Section 7. Section ?? concludes.

\section{Data structures}

Total Factor Productivity measures the temporal change in the absolute and relative productive performance of a set of $k=1,2, \ldots, K$ observations on DMUs (decision making units such as plants, firms, industries, economies) over $t=0,1, \ldots, T$ time periods. In period $t$ these DMUs transform a vector of $n=1,2, \ldots, N$ inputs $\boldsymbol{x}^{t} \in R_{++}^{N}$ into a vector of $m=1,2, \ldots, M$ outputs $\boldsymbol{y}^{t} \in \mathbb{R}_{++}^{M}$. Piecewise linear approximations of the technology can be obtained through Data Envelopment Analysis techniques. Specifically, the constant returns to scale (CRS) production possibility set introduced by Charnes, Cooper, and Rhodes (1978), known as the CCR model, corresponds to: $\check{S}^{t}=\left\{\left(\boldsymbol{x}^{t}, \boldsymbol{y}^{t}\right) \mid \mathbf{x}^{t} \geqslant X^{t} \lambda, \boldsymbol{y}^{t} \leqslant Y^{t} \boldsymbol{\lambda}, \boldsymbol{\lambda} \geqslant \mathbf{0}\right\}$,

\footnotetext{
${ }^{1}$ The address of the repository is https://github.com/joselzofio/TFPMATLAB.
} 
where $X^{t}=\left(\boldsymbol{x}_{i}^{t}\right) \in \mathbb{R}^{N \times K}$ and $Y^{t}=\left(\boldsymbol{y}_{i}^{t}\right) \in \mathbb{R}^{M \times K}$ are matrices, and $\lambda=\left(\lambda_{1}, \ldots, \lambda_{K}\right)^{\prime}$ is a semipositive vector. Alternatively, the variable returns to scale (VRS) production possibility set introduced by Banker, Charnes, and Cooper (1984) (BCC), corresponds to $S^{t}=$ $\left\{\left(\boldsymbol{x}^{t}, \boldsymbol{y}^{t}\right) \mid \boldsymbol{x}^{t} \geqslant X^{t} \boldsymbol{\lambda}, \boldsymbol{y}^{t} \leqslant Y^{t} \boldsymbol{\lambda}, \boldsymbol{e} \boldsymbol{\lambda}=\mathbf{1}, \boldsymbol{\lambda} \geqslant \mathbf{0}\right\}$, where $\boldsymbol{e}$ is a row vector with all elements equal to 1 . Therefore, the only difference with the CRS model is the adjunction of the condition $\sum_{k=1}^{K} \lambda_{k}=1$. Now, if input and output prices are available: $\boldsymbol{w}^{t} \in \mathbb{R}_{++}^{N}$ and $\boldsymbol{p}^{t} \in \mathbb{R}_{++}^{M}$, each observation $k$ is represented by the following panel data vector $\left(\boldsymbol{w}_{k}^{t}, \boldsymbol{x}_{k}^{t}, \boldsymbol{p}_{k}^{t}, \boldsymbol{y}_{k}^{t}\right)$. Data are managed as regular MATLAB vectors and matrices, constituting the inputs of the estimation functions.

In this paper we illustrate our work with data consisting of five DMUs with two inputs, two outputs, and two time periods. Inputs and outputs must be stored in three-dimensional (3D) arrays in which the first dimension (rows) correspond to the DMUs, the second dimension (column) to the input or the output number, and the third dimension to the time period. Input and output prices could be equal or different among DMUs. At least two time periods are required in order to compute Total Factor Productivity measures. The code for preparing the data in MATLAB for this paper is the following:

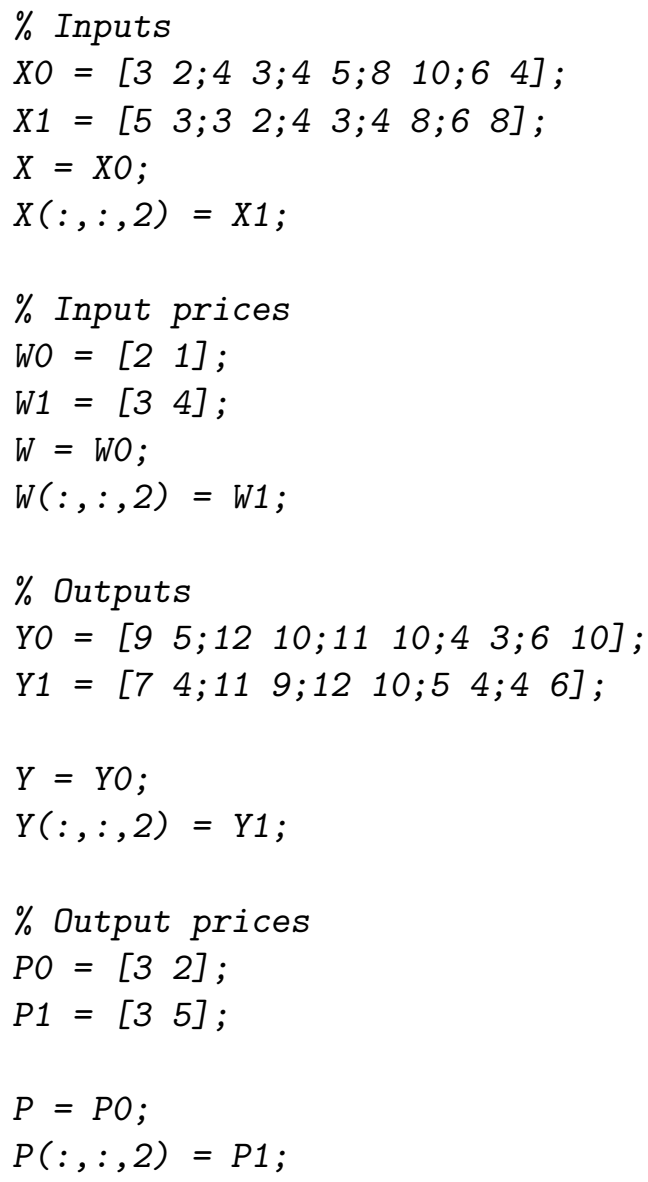

All estimation functions return a structure tfpout that contains fields with the estimation results as well as the input of the estimation function. Fields can be accessed directly using the dot notation, and the whole structure can be used as an input to other functions that print or export results (e.g., tfpdisp). 
Some of the fields of the tfpout structure are the following: ${ }^{2}$

- $\mathrm{X}$ and Y: Contain the input and output quantities, respectively.

- $\mathrm{W}$ and P: Contain the input and output prices, respectively.

- $\mathrm{K}$ : number of observations.

- $\mathrm{N}$ and M: Number of input and output variables, respectively.

- orient, period, decomp: Strings containing the orientation of the TFP model, reference period, and decomposition depending on scale and mix effects assumptions.

- tfp.M, tfp.MB, tfp.PROD, and tfp.GPROD: Computed Malmquist, Moorsteen-Bjurek, price-weighted, and share-weighted productivity indices.

- tfp.EC, and tfp.TC: Computed technical efficiency change and technological change factors.

- tfp.SEC, tfp.OME, tfp.IME and tfp.RTS: Computed scale effect, output mix effect, input mix effect, and returns to scale factors.

- names: Names of the DMUs.

\section{Malmquist Productivity Index}

If only quantities are available the most popular definition of TFP corresponds to the Malmquist productivity index (herefafter MPI). The MPI requires calculation of the output - or input - orientated distance function of observation $\left(\boldsymbol{x}_{k}^{t}, \boldsymbol{y}_{k}^{t}\right)$ observed in any two consecutive periods; i.e., the base period $t=0$ and the comparison period $t=1$, with respect to the constant returns to scale reference frontier corresponding to either $t=0$ or $t=1$. The constant returns benchmark is necessary for the distance function to satisfy the homogeneity conditions that ensure that the MPI fulfils the required proportionality properties (Balk and Zofío (2018)). Both distance functions can be interpreted as measures of technical efficiency with respect to either technology.

By taking the (constant-returns-to-scale, CRS, or cone) technology in the base period, $\check{S}^{t}$, as reference, and dropping the subscript $k$ identifying a specific observation for convenience, the output distance function is defined by $\check{D}_{o}^{t}\left(\boldsymbol{x}^{t}, \boldsymbol{y}^{t}\right) \equiv \inf \left\{\delta \mid \delta>0,\left(\boldsymbol{x}^{t}, \boldsymbol{y}^{t} / \delta\right) \in \check{S}^{t}\right\}$. Operationally, within the DEA framework, it can be calculated by solving the following program: $\check{D}_{o}^{t}\left(\boldsymbol{x}^{t}, \boldsymbol{y}^{t}\right)^{-1} \equiv \max _{\check{\phi}, \lambda}\left\{\check{\phi} \mid \boldsymbol{x}^{t} \geqslant X^{t} \lambda, \boldsymbol{y}^{t} \check{\phi} \leqslant Y^{t} \boldsymbol{\lambda}, \boldsymbol{\lambda} \geqslant \mathbf{0}\right\}$, with the optimal solution being denoted by $\check{\phi}^{*}$. Thus, $\left(\boldsymbol{x}^{t}, \boldsymbol{y}^{t} / \check{D}_{o}^{t}\left(\boldsymbol{x}^{t}, \boldsymbol{y}^{t}\right)\right)=\left(\boldsymbol{x}^{t}, \boldsymbol{y}^{t} \check{\phi}^{*}\right)$ is the point on the frontier of the period $t$ technology that is obtained by holding the input quantity vector $x$ constant while radially expanding the output quantity vector $\boldsymbol{y}^{t}$.

Alternatively, the input distance function is defined as $\check{D}_{i}^{t}\left(\boldsymbol{x}^{t}, \boldsymbol{y}^{t}\right) \equiv \sup \left\{\delta \mid \delta>0,\left(\boldsymbol{x}^{t} / \delta, \boldsymbol{y}^{t}\right) \in\right.$ $\left.\check{S}^{t}\right\}$. In this case it can be calculated by solving the program: $\check{D}_{i}^{t}\left(\boldsymbol{x}^{t}, \boldsymbol{y}^{t}\right)^{-1} \equiv \min _{\breve{\theta}, \lambda}\left\{\check{\theta} \mid \mathbf{x}^{t} \check{\theta} \geqslant\right.$ $\left.X^{t} \lambda, \boldsymbol{y}^{t} \leqslant Y^{t} \boldsymbol{\lambda}, \boldsymbol{\lambda} \geqslant \mathbf{0}\right\}$, and $\check{\theta}^{*}$ denotes the optimal solution. Thus, $\left(\boldsymbol{x}^{t} / \check{D}_{i}^{t}\left(\boldsymbol{x}^{t}, \boldsymbol{y}^{t}\right), \boldsymbol{y}^{t}\right)=$

\footnotetext{
${ }^{2}$ For a full list see the help of the function typing help tfpout in MATLAB.
} 
$\left(\boldsymbol{x}^{t} \check{\theta}^{*}, \boldsymbol{y}^{t}\right)$ is the point on the frontier of the period $t$ technology that is obtained by holding the output quantity vector $\boldsymbol{y}^{t}$ constant while radially contracting the input quantity vector $\boldsymbol{x}^{t}$.

In both cases, the variable-returns-to-scale (VRS) counterparts of the above output and input distance functions, defined on the actual technology $S^{t}$, and denoted by $D_{o}^{t}\left(\boldsymbol{x}^{t}, \boldsymbol{y}^{t}\right)$ and $D_{i}^{t}\left(\boldsymbol{x}^{t}, \boldsymbol{y}^{t}\right)$, are solved in the same way, with $\boldsymbol{e} \boldsymbol{\lambda}=\mathbf{1}$ as additional constraint.

\subsection{The output orientated MPI}

The output orientated MPI, conditional on the period $t$ constant returns to scale technology, is defined by

$$
\check{M}_{o}^{t}\left(\boldsymbol{x}^{1}, \boldsymbol{y}^{1}, \boldsymbol{x}^{0}, \boldsymbol{y}^{0}\right) \equiv \frac{\check{D}_{o}^{t}\left(\boldsymbol{x}^{1}, \boldsymbol{y}^{1}\right)}{\check{D}_{o}^{t}\left(\boldsymbol{x}^{0}, \boldsymbol{y}^{0}\right)}
$$

Selecting the base period technology then leads to $\check{M}_{o}^{0}\left(x^{1}, y^{1}, x^{0}, y^{0}\right)$; selecting the comparison period technology leads to $\check{M}_{o}^{1}\left(x^{1}, y^{1}, x^{0}, y^{0}\right)$. The TFP toolbox calculates both, as well as their geometric mean. Let us start with the first option.

\section{The output orientated, base period MPI}

Following Balk and Zofío (2018), who provide meaningful theoretical interpretations for the different factors, the first extended decomposition of the output orientated-base period MPI (termed 'Path A' by these authors relying on its geometric representation in the single-output single-input case-see their Figure 1 for visual guidance), corresponds to

$$
\begin{aligned}
\check{M}_{o}^{0}\left(\boldsymbol{x}^{1}, \boldsymbol{y}^{1}, \boldsymbol{x}^{0}, \boldsymbol{y}^{0}\right)= & E C_{o}\left(\boldsymbol{x}^{1}, \boldsymbol{y}^{1}, \boldsymbol{x}^{0}, \boldsymbol{y}^{0}\right) \times T C_{o}^{1,0}\left(\boldsymbol{x}^{1}, \boldsymbol{y}^{1}\right) \times \\
& S E C_{o}^{0}\left(\boldsymbol{x}^{1}, \boldsymbol{x}^{0}, \boldsymbol{y}^{0}\right) \times O M E^{0}\left(\boldsymbol{x}^{1}, \boldsymbol{y}^{1}, \boldsymbol{y}^{0}\right) .
\end{aligned}
$$

In this expression, initially presented by Balk (2001), there are four mutually independent factors, with the following interpretation:

- Efficiency change: $E C_{o}\left(\boldsymbol{x}^{1}, \boldsymbol{y}^{1}, \boldsymbol{x}^{0}, \boldsymbol{y}^{0}\right)=D_{o}^{1}\left(\boldsymbol{x}^{1}, \boldsymbol{y}^{1}\right) / D_{o}^{0}\left(\boldsymbol{x}^{0}, \boldsymbol{y}^{0}\right)$, representing the change in the technical efficiency of the observation under evaluation with respect to the two periods, i.e., the catch-up effect.

- Technological change: $T C_{o}^{1,0}\left(\boldsymbol{x}^{1}, \boldsymbol{y}^{1}\right)=D_{o}^{0}\left(\boldsymbol{x}^{1}, \boldsymbol{y}^{1}\right) / D_{o}^{1}\left(\boldsymbol{x}^{1}, \boldsymbol{y}^{1}\right)$, capturing the change in the reference frontier between both periods, i.e., the frontier-shift effect.

- Radial scale and input mix effect: $S E C_{o}^{0}\left(\boldsymbol{x}^{1}, \boldsymbol{x}^{0}, \boldsymbol{y}^{0}\right)=\left[\check{D}_{o}^{0}\left(\boldsymbol{x}^{1}, \boldsymbol{y}^{0}\right) / D_{o}^{0}\left(\boldsymbol{x}^{1}, \boldsymbol{y}^{0}\right)\right] \times$ $\left[D_{o}^{0}\left(\boldsymbol{x}^{0}, \boldsymbol{y}^{0}\right) / \check{D}_{o}^{0}\left(\boldsymbol{x}^{0}, \boldsymbol{y}^{0}\right)\right]$, corresponding to scale efficiency improvements associated to radial increases in the inputs, and the additional scale efficiency change originating from changes in the input mix. ${ }^{3}$

\footnotetext{
${ }^{3}$ Balk and Zofío (2018) show that $S E C_{o}^{0}\left(\boldsymbol{x}^{1}, \boldsymbol{x}^{0}, \boldsymbol{y}^{0}\right)$ can be further decomposed into a radial scale effect: $S E C_{o}^{0}\left(\lambda \boldsymbol{x}^{0}, \boldsymbol{x}^{0}, \boldsymbol{y}^{0}\right)=\left[\check{D}_{o}^{0}\left(\lambda \boldsymbol{x}^{0}, \boldsymbol{y}^{0}\right) / D_{o}^{0}\left(\lambda \boldsymbol{x}^{0}, \boldsymbol{y}^{0}\right)\right] \times\left[D_{o}^{0}\left(\boldsymbol{x}^{0}, \boldsymbol{y}^{0}\right) / \check{D}_{o}^{0}\left(\boldsymbol{x}^{0}, \boldsymbol{y}^{0}\right)\right]$, and an input mix effect: $S E C_{o}^{0}\left(\boldsymbol{x}^{1}, \lambda \boldsymbol{x}^{0}, \boldsymbol{y}^{0}\right)=\left[\check{D}_{o}^{0}\left(\boldsymbol{x}^{1}, \boldsymbol{y}^{0}\right) / D_{o}^{0}\left(\boldsymbol{x}^{1}, \boldsymbol{y}^{0}\right)\right] \times\left[D_{o}^{0}\left(\lambda \boldsymbol{x}^{0}, \boldsymbol{y}^{0}\right) / \check{D}_{o}^{0}\left(\lambda \boldsymbol{x}^{0}, \boldsymbol{y}^{0}\right)\right]$. Implementing this extended decomposition requires choosing a suitable value for $\lambda$, for instance such that $D_{o}^{0}\left(\lambda \boldsymbol{x}^{0}, \boldsymbol{y}^{0}\right)=D_{o}^{0}\left(\boldsymbol{x}^{1}, \boldsymbol{y}^{0}\right)$, which means that $\lambda \boldsymbol{x}^{0}$ and $\boldsymbol{x}^{1}$ are on the same output isoquant of the base period technology. Unfortunately, solving this equation in a DEA framework is so complicated that it is impractical from an applied perspective. Moreover, the existence of a solution under VRS is not guaranteed due to infeasibilities.
} 
- Output mix effect: $O M E^{0}\left(\boldsymbol{x}^{1}, \boldsymbol{y}^{1}, \boldsymbol{y}^{0}\right)=\left[\check{D}_{o}^{0}\left(\boldsymbol{x}^{1}, \boldsymbol{y}^{1}\right) / D_{o}^{0}\left(\boldsymbol{x}^{1}, \boldsymbol{y}^{1}\right)\right] \times\left[D_{o}^{0}\left(\boldsymbol{x}^{1}, \boldsymbol{y}^{0}\right) /\right.$ $\left.\check{D}_{o}^{0}\left(\boldsymbol{x}^{1}, \boldsymbol{y}^{0}\right)\right]$, showing the counterpart scale efficiency effects associated to changes in the output mix.

Factors with values greater than 1 contribute to productivity growth (e.g., through technical efficiency gains or technological progress), while factors whose values are smaller than 1 are detrimental.

An alternative decomposition of the base period, output orientated MPI reverses the order in which changes in the input and output space take place in the last two factors in expression (3). This yields

$$
\begin{aligned}
\check{M}_{o}^{0}\left(\boldsymbol{x}^{1}, \boldsymbol{y}^{1}, \boldsymbol{x}^{0}, \boldsymbol{y}^{0}\right)= & E C_{o}\left(\boldsymbol{x}^{1}, \boldsymbol{y}^{1}, \boldsymbol{x}^{0}, y^{0}\right) \times T C_{o}^{1,0}\left(\boldsymbol{x}^{1}, \boldsymbol{y}^{1}\right) \times \\
& S E C_{o}^{0}\left(\boldsymbol{x}^{1}, \boldsymbol{x}^{0}, \boldsymbol{y}^{1}\right) \times O M E^{0}\left(\boldsymbol{x}^{0}, \boldsymbol{y}^{1}, \boldsymbol{y}^{0}\right),
\end{aligned}
$$

which is termed 'Path B' by the same authors. The differences between this decomposition and that in expression (2) are subtle but noteworthy. The parts capturing efficiency change and technological change are identical - whose product corresponds to the Malmquist index introduced by Caves et al. (1982) as we remark later. The factor capturing the radial scale effect and the input mix effect is conditional on $\boldsymbol{y}^{0}$ in expression (2) but on $\boldsymbol{y}^{1}$ in expression (3). The reverse happens with the output mix effect; this effect is conditional on $\boldsymbol{x}^{1}$ in expression (2) but on $\boldsymbol{x}^{0}$ in expression (3).

Consequently, there are two, equally meaningful, decompositions of the Malmquist productivity index $\check{M}_{o}^{0}\left(x^{1}, y^{1}, x^{0}, y^{0}\right)$. As they differ only with respect to the factor jointly measuring the radial scale effect and the input mix effect and the factor measuring the output mix effect, it possible to take their geometric mean of expressions (2) and (3), thereby obtaining a final decomposition:

$$
\begin{aligned}
\check{M}_{o}^{0}\left(\boldsymbol{x}^{1}, \boldsymbol{y}^{1}, \boldsymbol{x}^{0}, \boldsymbol{y}^{0}\right)= & M_{o}^{0}\left(\boldsymbol{x}^{1}, \boldsymbol{y}^{1}, \boldsymbol{x}^{0}, \boldsymbol{y}^{0}\right) \times \\
& {\left[S E C_{o}^{0}\left(\boldsymbol{x}^{1}, \boldsymbol{x}^{0}, \boldsymbol{y}^{0}\right) \times S E C_{o}^{0}\left(\boldsymbol{x}^{1}, \boldsymbol{x}^{0}, \boldsymbol{y}^{1}\right)\right]^{1 / 2} \times } \\
& {\left[O M E^{0}\left(\boldsymbol{x}^{0}, \boldsymbol{y}^{1}, \boldsymbol{y}^{0}\right) \times O M E^{0}\left(\boldsymbol{x}^{1}, \boldsymbol{y}^{1}, \boldsymbol{y}^{0}\right)\right]^{1 / 2} . }
\end{aligned}
$$

To compute the MPI in MATLAB the user calls the deatfpm (X, Y, ...) function with: i) the orient parameter set to the output orientation (oo); 2) the period parameter set to (base); and iii) the decomposition parameter decomp set to (complete). ${ }^{4}$

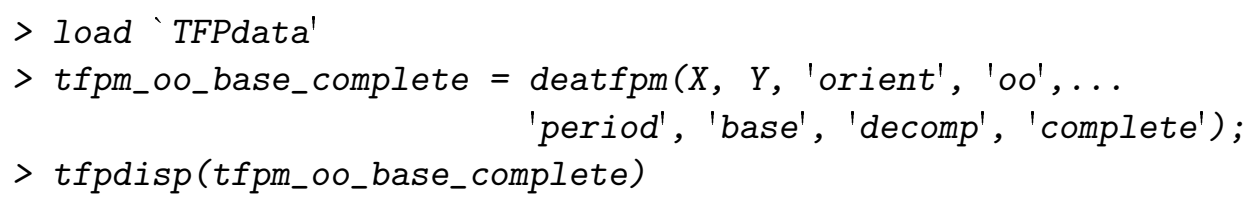

Total Factor Productivity (TFP)

\footnotetext{
${ }^{4}$ See Section 7.2 for advanced uses of the tfpdisp function.
} 


\begin{abstract}
DMUs : 5
Inputs: 2 Outputs: 2

Model: tfp-m

Orientation: oo (Output orientated)

Returns to scale: vrs (Variable)

Malmquist:

Reference period is base period

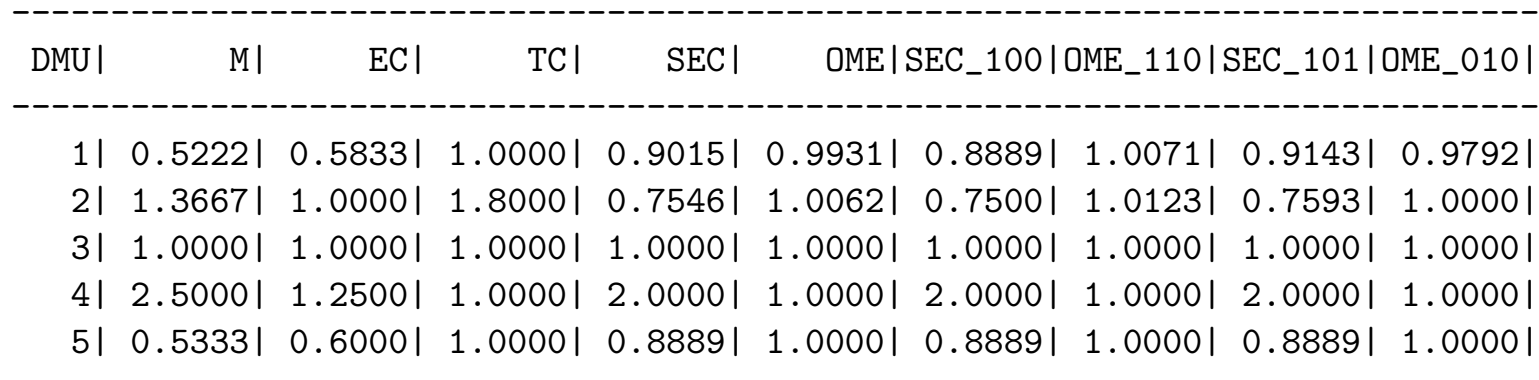

$\mathrm{M}=$ Malmquist. $\mathrm{EC}=$ Efficiency Change. $\mathrm{TC}=$ Technological Change.

SEC = Scale Effect. OME = Output Mix Effect.
\end{abstract}

The output of the function successively shows the MPI (M), the technical efficiency change factor (EC), the technological change factor (TC), the geometric mean of the scale effect factors (SEC), and that of the output mix effect factors (OME), and the values of the two last factors corresponding to 'Path A' and 'Path B' above - identified by the time superscripts of each of the input and output arguments in each factor, e.g., SEC_100 corresponds to $S E C_{o}^{0}\left(\boldsymbol{x}^{1}, \boldsymbol{x}^{0}, \boldsymbol{y}^{0}\right)$ in (2) and (4) above.

\title{
Related decompositions of the output orientated, base period MPI
}

The above four factor decompositions can be related to simpler proposals in the literature. First, they generalize the earlier proposal by Ray and Desli (1997). By merging the scale effect and the output mix effect in (2) or (3), both decompositions reduce to the same three-factor decomposition:

$$
\check{M}_{o}^{0}\left(\boldsymbol{x}^{1}, \boldsymbol{y}^{1}, \boldsymbol{x}^{0}, \boldsymbol{y}^{0}\right)=E C_{o}\left(\boldsymbol{x}^{1}, \boldsymbol{y}^{1}, \boldsymbol{x}^{0}, \boldsymbol{y}^{0}\right) \times T C_{o}^{1,0}\left(\boldsymbol{x}^{1}, \boldsymbol{y}^{1}\right) \times \frac{O S E^{0}\left(\boldsymbol{x}^{1}, \boldsymbol{y}^{1}\right)}{O S E^{0}\left(\boldsymbol{x}^{0}, \boldsymbol{y}^{0}\right)}
$$

where $O S E^{0}\left(\boldsymbol{x}^{1}, \boldsymbol{y}^{1}\right) / O S E^{0}\left(\boldsymbol{x}^{0}, \boldsymbol{y}^{0}\right)=\left[\check{D}_{o}^{0}\left(\boldsymbol{x}^{1}, \boldsymbol{y}^{1}\right) / D_{o}^{0}\left(\boldsymbol{x}^{1}, \boldsymbol{y}^{1}\right)\right] \times\left[D_{o}^{0}\left(\boldsymbol{x}^{0}, \boldsymbol{y}^{0}\right) / \check{D}_{o}^{0}\left(\boldsymbol{x}^{0}, \boldsymbol{y}^{0}\right)\right]$. Lovell (2003) suggests that this factor measures the contribution of returns to scale to productivity change. Following this interpretation, if it is greater than 1, increasing returns to scale result in productivity growth, while if it is smaller than 1, decreasing returns to scale are detrimental. If equal to one, constant returns to scale prevail for the observed input-output values. To obtain this simpler decomposition, the syntax is the same as in the extended one but with the parameter decomp set to (rts). In the output table the column RTS contains the contribution of returns to scale to productivity change. 


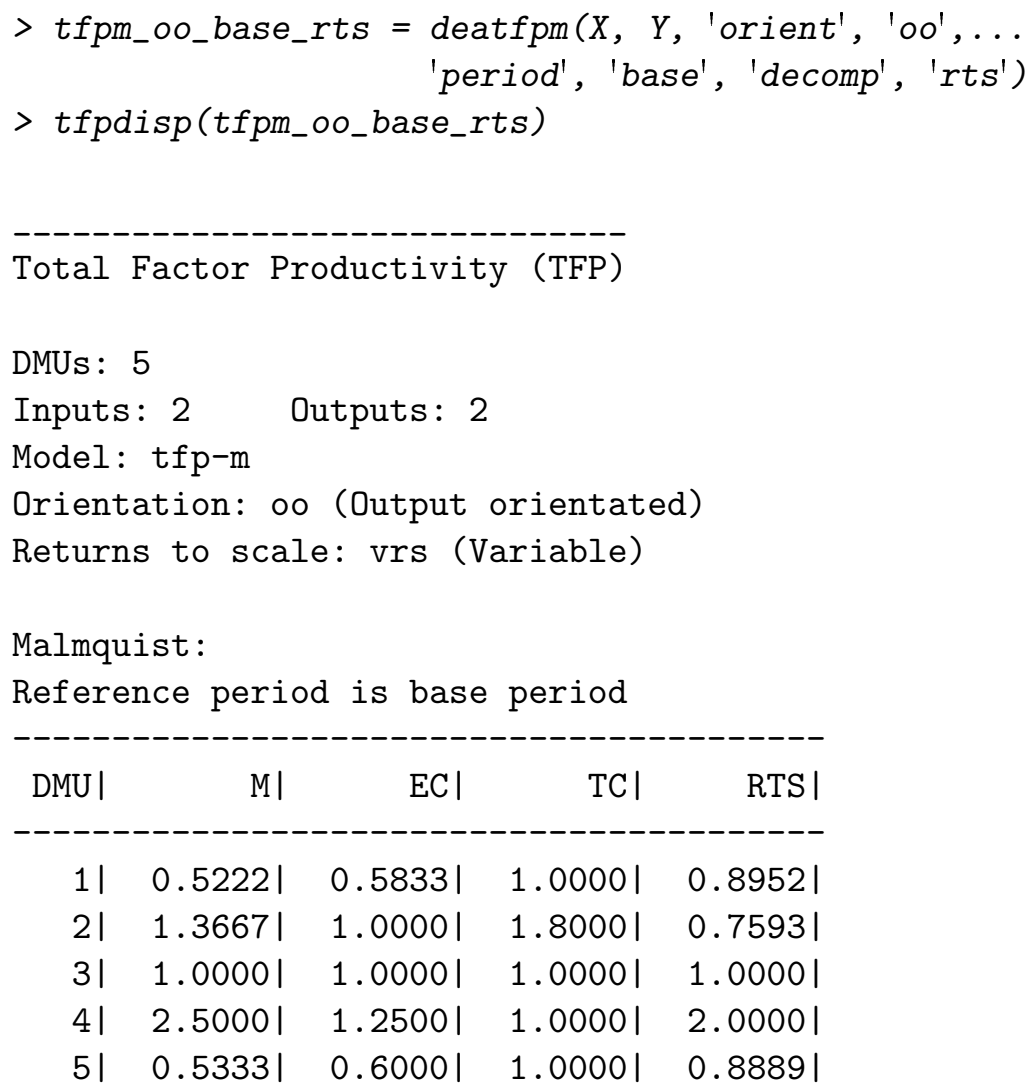

$M=$ Malmquist. $E C=$ Efficiency Change. $T C=$ Technological Change.

RTS $=$ Returns to Scale.

Second, the technical efficiency change and technological change terms common to expressions (2) and (3) correspond to the base period, output orientated index proposed by Caves et al. (1982) $(C C D)$,

$$
M_{o}^{0}\left(\boldsymbol{x}^{1}, \boldsymbol{y}^{1}, \boldsymbol{x}^{0}, \boldsymbol{y}^{0}\right) \equiv E C_{o}\left(\boldsymbol{x}^{1}, \boldsymbol{y}^{1}, \boldsymbol{x}^{0}, \boldsymbol{y}^{0}\right) \times T C_{o}^{1,0}\left(\boldsymbol{x}^{1}, \boldsymbol{y}^{1}\right)=\frac{D_{o}^{0}\left(\boldsymbol{x}^{1}, \boldsymbol{y}^{1}\right)}{D_{o}^{0}\left(\boldsymbol{x}^{0}, \boldsymbol{y}^{0}\right)}
$$

Substituting (6) in any of the above decompositions yields alternative expressions, e.g., for (2) and (3):

$$
\check{M}_{o}^{0}\left(\boldsymbol{x}^{1}, \boldsymbol{y}^{1}, \boldsymbol{x}^{0}, \boldsymbol{y}^{0}\right)=M_{o}^{0}\left(\boldsymbol{x}^{1}, \boldsymbol{y}^{1}, \boldsymbol{x}^{0}, \boldsymbol{y}^{0}\right) \times S E C_{o}^{0}\left(\boldsymbol{x}^{1}, \boldsymbol{x}^{0}, \boldsymbol{y}^{0}\right) \times O M E^{0}\left(\boldsymbol{x}^{1}, \boldsymbol{y}^{1}, \boldsymbol{y}^{0}\right),
$$

and

$$
\check{M}_{o}^{0}\left(\boldsymbol{x}^{1}, \boldsymbol{y}^{1}, \boldsymbol{x}^{0}, \boldsymbol{y}^{0}\right)=M_{o}^{0}\left(\boldsymbol{x}^{1}, \boldsymbol{y}^{1}, \boldsymbol{x}^{0}, \boldsymbol{y}^{0}\right) \times S E C_{o}^{0}\left(\boldsymbol{x}^{1}, \boldsymbol{x}^{0}, \boldsymbol{y}^{1}\right) \times O M E^{0}\left(\boldsymbol{x}^{0}, \boldsymbol{y}^{1}, \boldsymbol{y}^{0}\right) .
$$

To obtain the complete decomposition showing the $C C D$ index rather than its individual components the parameter decomp must be changed to (ccd). 


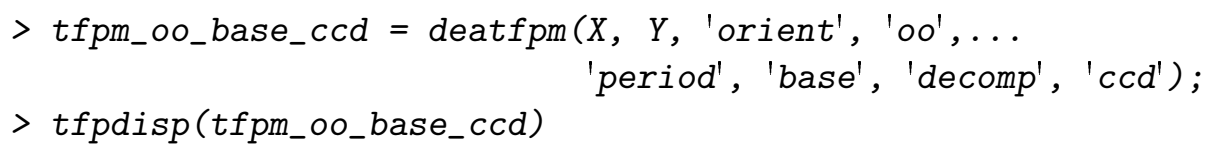

Total Factor Productivity (TFP)

DMUs : 5

Inputs: 2 Outputs: 2

Model: tfp-m

Orientation: oo (Output orientated)

Returns to scale: vrs (Variable)

Malmquist:

Reference period is base period

\begin{tabular}{|c|c|c|c|c|c|c|c|c|}
\hline DMU I & $\mathrm{Ml}$ & Mvrs | & $\mathrm{SECl}$ & OME I & SEC_100I & OME_110I & SEC_101I & OME_010I \\
\hline 11 & .52221 & 31 & $.9015 \mid$ & 1 & $0 . \varepsilon$ & 1.0 & $0 . \varsigma$ & 0.9 \\
\hline 21 & $1.3667 \mid$ & 1.80001 & $0.7546 \mid$ & 1.00621 & 0.75001 & $1.0123 \mid$ & 0.7 & 1.00001 \\
\hline 31 & 1.00001 & 1.00001 & 1.00001 & 1.00001 & 1.00001 & 1.00001 & 1.00001 & 1.00001 \\
\hline 41 & 2.50001 & $1.2500 \mid$ & 2.00001 & 1.00001 & 2.00001 & 1.00001 & 2.0 & 1.00001 \\
\hline 51 & 0.53331 & 0.60001 & $0.8889 \mid$ & 1.00001 & $0.8889 \mid$ & 1.00001 & $0.8889 \mid$ & 1.00001 \\
\hline
\end{tabular}

$M=$ Malmquist. Mvrs = Malmquist VRS. SEC = Scale Effect.

OME = Output Mix Effect.

In the extant literature on productivity measurement, the $C C D$ index frequently figures under the name 'the (input orientated) Malmquist productivity index'. However, the $C C D$ index (6) cannot be regarded as a productivity index because it does not satisfy the proportionality property, as initially shown by Grifell-Tatjé and Lovell (1995), unless the technology exhibits constant returns to scale. In that specific case, all terms to the right of the $C C D$ index in expressions (7) and (8) become equal to 1 , and, consequently, $\check{M}_{o}^{0}\left(x^{1}, y^{1}, x^{0}, y^{0}\right)$ $=M_{o}^{0}\left(x^{1}, y^{1}, x^{0}, y^{0}\right)$. Decomposing the $C C D$ is then accomplished by assuming constant returns to scale, as initially proposed by Färe, Grosskopf, Lindgren, and Roos (1994), resulting in

$$
\check{M}_{o}^{0}\left(\boldsymbol{x}^{1}, \boldsymbol{y}^{1}, \boldsymbol{x}^{0}, \boldsymbol{y}^{0}\right)=\check{E C_{o}}\left(\boldsymbol{x}^{1}, \boldsymbol{y}^{1}, \boldsymbol{x}^{0}, \boldsymbol{y}^{0}\right) \times \check{T C_{o}^{1,0}}\left(\boldsymbol{x}^{1}, \boldsymbol{y}^{1}\right)
$$

Therefore, prior to decomposing the MPI under wrong returns to scale assumptions, it is advisable to test whether constant returns are statistically supported by the data. In this scheme, the DEA distance function measures can be regarded as estimators of their true values. Simar and Wilson (1998) introduce bootstrap methods that, based on resampling, provide estimation bias, confidence intervals, and allow hypotheses testing. This toolbox implements the algorithms presented by Simar and Wilson (2002), following Bogetoft and Otto (2011), to determine whether the constant and variable returns distance function values are significantly different or not. The returns to scale test for all $t=0,1,2, \ldots, T$ periods can 
be performed by calling the deatestrtsm (X, Y, ...) function, with the orient parameter set to the same orientation as the MPI, i.e., (oo) in the present case. It is also possible to change the number of bootstrap replications set in the nreps parameter (200 by default), as well as the default 5\% significance level in the alpha parameter (0.05). Results of the test can be displayed on the screen by setting the parameter disp to 1 .

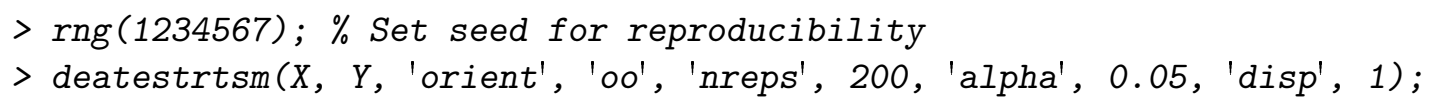

If the null hypothesis cannot be rejected, the two-factor decomposition of the MPI under constant returns to scale (9) is warranted, and can be calculated with the toolbox by setting the parameter decomp to (crs).

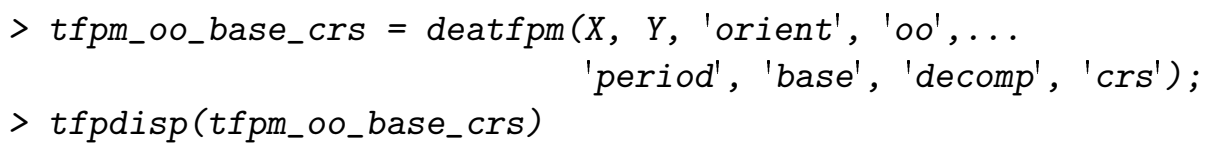

Total Factor Productivity (TFP)

DMUs : 5

Inputs: 2 Outputs: 2

Model: tfp-m

Orientation: oo (Output orientated)

Returns to scale: crs (Constant)

Malmquist:

Reference period is base period 


\begin{tabular}{|c|c|c|c|}
\hline DMU I & $\mathrm{M} \mid$ & $\mathrm{ECl}$ & $\mathrm{TCl}$ \\
\hline 11 & 0.52221 & 0.42421 & 1.2310 \\
\hline 21 & $1.3667 \mid$ & 1.00001 & $1.3667 \mid$ \\
\hline 31 & 1.00001 & 0.83331 & 1.2000 \\
\hline 41 & 2.50001 & $2.0455 \mid$ & 1.2222 \\
\hline 51 & 0.53331 & 0.44441 & 1.20001 \\
\hline
\end{tabular}

$\mathrm{M}=$ Malmquist. $\mathrm{EC}=$ Efficiency Change. $\mathrm{TC}=$ Technological Change.

The output orientated, comparison period MPI

As anticipated, we can consider the comparison period technology, $\check{S}^{1}$, as benchmark. The comparison period, output orientated MPI is then definned by $\check{M}_{o}^{1}\left(\boldsymbol{x}^{1}, \boldsymbol{y}^{1}, \boldsymbol{x}^{0}, \boldsymbol{y}^{0}\right)$. The complete four-factor decomposition counterparts to expressions (2) and (3) are, respectively,

$$
\begin{aligned}
\check{M}_{o}^{1}\left(\boldsymbol{x}^{1}, \boldsymbol{y}^{1}, \boldsymbol{x}^{0}, y^{0}\right)= & E C_{o}\left(\boldsymbol{x}^{1}, \boldsymbol{y}^{1}, \boldsymbol{x}^{0}, \boldsymbol{y}^{0}\right) \times T C_{o}^{1,0}\left(\boldsymbol{x}^{0}, \boldsymbol{y}^{0}\right) \times \\
& S E C_{o}^{1}\left(\boldsymbol{x}^{1}, \boldsymbol{x}^{0}, \boldsymbol{y}^{0}\right) \times O M E^{1}\left(\boldsymbol{x}^{1}, \boldsymbol{y}^{1}, \boldsymbol{y}^{0}\right),
\end{aligned}
$$

and

$$
\begin{aligned}
\check{M}_{o}^{1}\left(\boldsymbol{x}^{1}, \boldsymbol{y}^{1}, \boldsymbol{x}^{0}, y^{0}\right)= & E C_{o}\left(\boldsymbol{x}^{1}, \boldsymbol{y}^{1}, \boldsymbol{x}^{0}, \boldsymbol{y}^{0}\right) \times T C_{o}^{1,0}\left(\boldsymbol{x}^{0}, \boldsymbol{y}^{0}\right) \times \\
& S E C_{o}^{1}\left(\boldsymbol{x}^{1}, \boldsymbol{x}^{0}, \boldsymbol{y}^{1}\right) \times O M E^{1}\left(\boldsymbol{x}^{0}, \boldsymbol{y}^{1}, \boldsymbol{y}^{0}\right),
\end{aligned}
$$

which are identified by 'Path C' and 'Path D' in Balk and Zofío (2018). Again, it is possible to take the geometric mean of these two decompositions, thereby obtaining

$$
\begin{aligned}
\check{M}_{o}^{1}\left(\boldsymbol{x}^{1}, \boldsymbol{y}^{1}, \boldsymbol{x}^{0}, \boldsymbol{y}^{0}\right)= & M_{o}^{1}\left(\boldsymbol{x}^{1}, \boldsymbol{y}^{1}, \boldsymbol{x}^{0}, \boldsymbol{y}^{0}\right) \times \\
& {\left[S E C_{o}^{1}\left(\boldsymbol{x}^{1}, \boldsymbol{x}^{0}, \boldsymbol{y}^{0}\right) \times S E C_{o}^{1}\left(\boldsymbol{x}^{1}, \boldsymbol{x}^{0}, \boldsymbol{y}^{1}\right)\right]^{1 / 2} \times } \\
& {\left[O M E^{1}\left(\boldsymbol{x}^{0}, \boldsymbol{y}^{1}, \boldsymbol{y}^{0}\right) \times O M E^{1}\left(\boldsymbol{x}^{1}, \boldsymbol{y}^{1}, \boldsymbol{y}^{0}\right)\right]^{1 / 2}, }
\end{aligned}
$$

where the comparison period, output orientated $C C D$ index is defined by

$$
M_{o}^{1}\left(\boldsymbol{x}^{1}, \boldsymbol{y}^{1}, \boldsymbol{x}^{0}, \boldsymbol{y}^{0}\right) \equiv E C_{o}\left(\boldsymbol{x}^{1}, \boldsymbol{y}^{1}, \boldsymbol{x}^{0}, \boldsymbol{y}^{0}\right) \times T C_{o}^{1,0}\left(\boldsymbol{x}^{0}, \boldsymbol{y}^{0}\right)=\frac{D_{o}^{1}\left(\boldsymbol{x}^{1}, \boldsymbol{y}^{1}\right)}{D_{o}^{1}\left(\boldsymbol{x}^{0}, \boldsymbol{y}^{0}\right)}
$$

The complete output orientated, comparison period MPI can be calculated in MATLAB by calling the deatfpm (X, Y, ...) function, but substituting (base) by (comparison) in the period parameter, and leaving the other parameters unchanged.

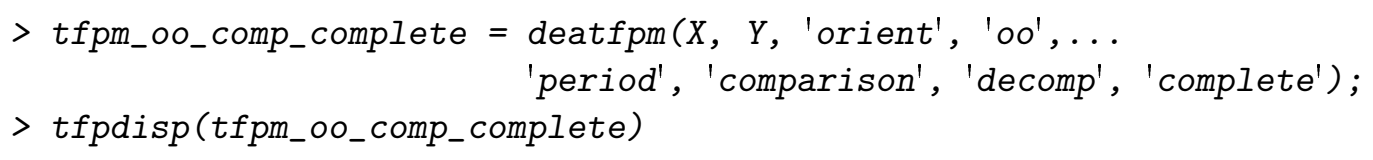




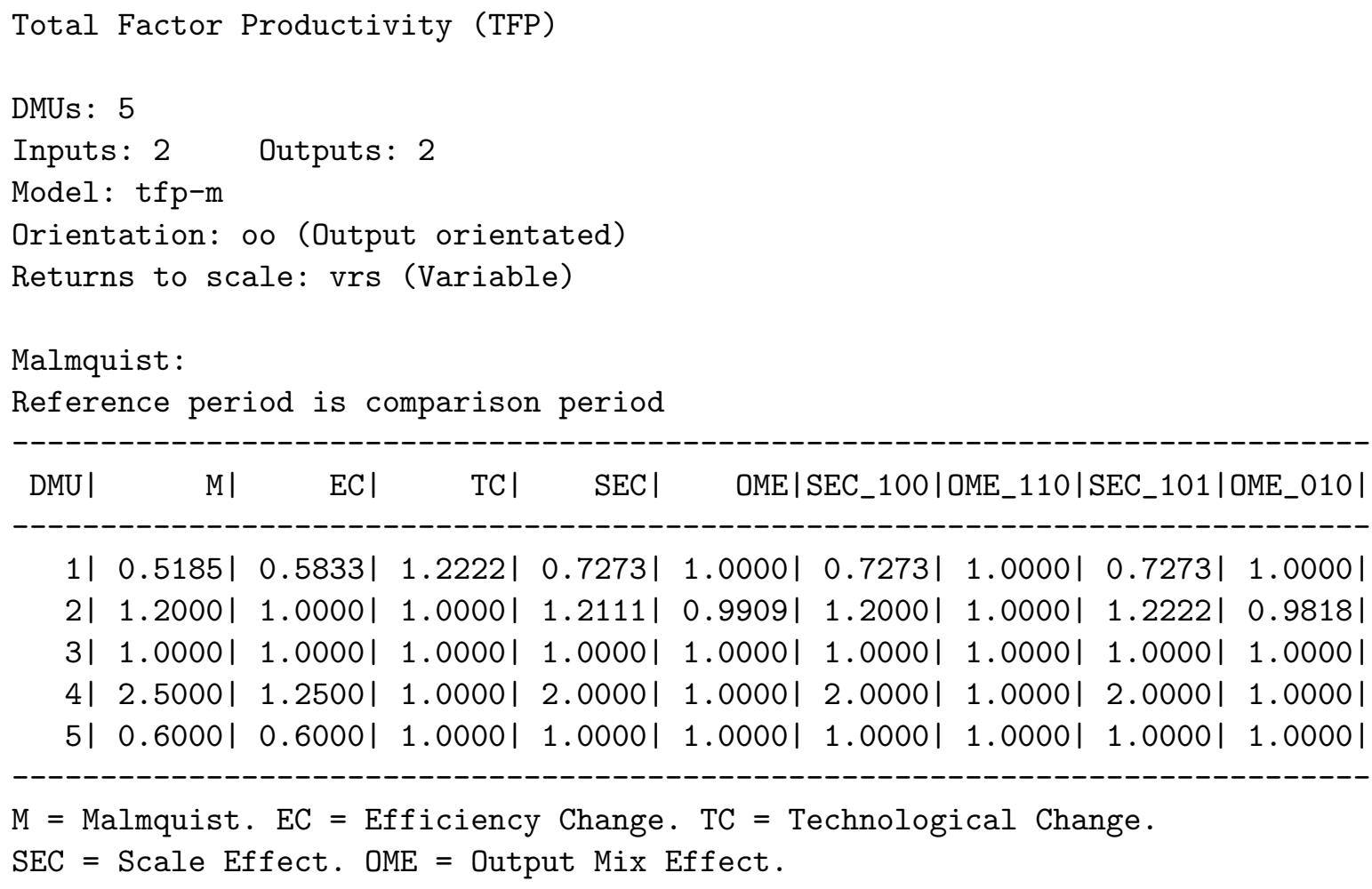

Once this change is made, obtaining the related decompositions can be easily done by setting the parameter decomp to either ( $\mathrm{rts}),(\mathrm{ccd})$, or (crs). The output of the function is interpreted in the same way as for its base period counterpart (4).

\section{The output orientated, geometric mean MPI}

The output orientated MPIs from the base and comparison period perspectives yield different results unless the cone technology based technological change exhibits neutrality. As this restrictive condition is generally unobserved, a compromise between the two periods can be reached by defining their geometric mean, i.e.,

$$
\begin{gathered}
\check{M}_{o}\left(x^{1}, y^{1}, x^{0}, y^{0}\right) \equiv\left[\check{M}_{o}^{0}\left(\boldsymbol{x}^{1}, \boldsymbol{y}^{1}, \boldsymbol{x}^{0}, \boldsymbol{y}^{0}\right) \times \check{M}_{o}^{1}\left(\boldsymbol{x}^{1}, \boldsymbol{y}^{1}, \boldsymbol{x}^{0}, \boldsymbol{y}^{0}\right)\right]^{1 / 2}= \\
{\left[\frac{\check{D}_{o}^{0}\left(\boldsymbol{x}^{1}, \boldsymbol{y}^{1}\right)}{\check{D}_{o}^{0}\left(\boldsymbol{x}^{0}, \boldsymbol{y}^{0}\right)} \frac{\check{D}_{o}^{1}\left(\boldsymbol{x}^{1}, \boldsymbol{y}^{1}\right)}{\check{D}_{o}^{1}\left(\boldsymbol{x}^{0}, \boldsymbol{y}^{0}\right)}\right]^{1 / 2} .}
\end{gathered}
$$

This index can be decomposed into the following factors,

$$
\begin{gathered}
\check{M}_{o}\left(\boldsymbol{x}^{1}, \boldsymbol{y}^{1}, \boldsymbol{x}^{0}, \boldsymbol{y}^{0}\right)= \\
{\left[M_{o}^{0}\left(\boldsymbol{x}^{1}, \boldsymbol{y}^{1}, \boldsymbol{x}^{0}, \boldsymbol{y}^{0}\right) M_{o}^{1}\left(\boldsymbol{x}^{1}, \boldsymbol{y}^{1}, \boldsymbol{x}^{0}, y^{0}\right)\right]^{1 / 2} \times} \\
{\left[S E C_{o}^{0}\left(\boldsymbol{x}^{1}, \boldsymbol{x}^{0}, \boldsymbol{y}^{0}\right) S E C_{o}^{0}\left(\boldsymbol{x}^{1}, \boldsymbol{x}^{0}, \boldsymbol{y}^{1}\right) S E C_{o}^{1}\left(\boldsymbol{x}^{1}, \boldsymbol{x}^{0}, \boldsymbol{y}^{0}\right) S E C_{o}^{1}\left(\boldsymbol{x}^{1}, \boldsymbol{x}^{0}, \boldsymbol{y}^{1}\right)\right]^{1 / 4} \times} \\
{\left[O M E^{0}\left(\boldsymbol{x}^{0}, \boldsymbol{y}^{1}, \boldsymbol{y}^{0}\right) O M E^{0}\left(\boldsymbol{x}^{1}, \boldsymbol{y}^{1}, \boldsymbol{y}^{0}\right) O M E^{1}\left(\boldsymbol{x}^{0}, \boldsymbol{y}^{1}, \boldsymbol{y}^{0}\right) O M E^{1}\left(\boldsymbol{x}^{1}, \boldsymbol{y}^{1}, \boldsymbol{y}^{0}\right)\right]^{1 / 4}=}
\end{gathered}
$$




$$
\begin{gathered}
{\left[M_{o}^{0}\left(\boldsymbol{x}^{1}, \boldsymbol{y}^{1}, \boldsymbol{x}^{0}, y^{0}\right) M_{o}^{1}\left(\boldsymbol{x}^{1}, \boldsymbol{y}^{1}, \boldsymbol{x}^{0}, \boldsymbol{y}^{0}\right)\right]^{1 / 2} \times} \\
{\left[\left[S E C_{o}^{0}\left(\boldsymbol{x}^{1}, \boldsymbol{x}^{0}, \boldsymbol{y}^{0}\right) S E C_{o}^{1}\left(\boldsymbol{x}^{1}, \boldsymbol{x}^{0}, \boldsymbol{y}^{0}\right)\right]^{1 / 2}\left[S E C_{o}^{0}\left(\boldsymbol{x}^{1}, \boldsymbol{x}^{0}, \boldsymbol{y}^{1}\right) S E C_{o}^{1}\left(\boldsymbol{x}^{1}, \boldsymbol{x}^{0}, \boldsymbol{y}^{1}\right)\right]^{1 / 2}\right]^{1 / 2} \times} \\
{\left[\left[O M E^{0}\left(\boldsymbol{x}^{0}, \boldsymbol{y}^{1}, \boldsymbol{y}^{0}\right) O M E^{1}\left(\boldsymbol{x}^{0}, \boldsymbol{y}^{1}, \boldsymbol{y}^{0}\right)\right]^{1 / 2}\left[O M E^{0}\left(\boldsymbol{x}^{1}, \boldsymbol{y}^{1}, \boldsymbol{y}^{0}\right) O M E^{1}\left(\boldsymbol{x}^{1}, \boldsymbol{y}^{1}, \boldsymbol{y}^{0}\right)\right]^{1 / 2}\right]^{1 / 2}}
\end{gathered}
$$

where the second decomposition was obtained by simply rearranging the first. This second decomposition shows what happens if factors with the same structure but referring to different periods, i.e., 'Path A' and 'Path C', and 'Path B' and 'Path D', are combined prior to the final aggregation.

Setting the period parameter to (geomean) in the deatfpm $(\mathrm{X}, \mathrm{Y}, \ldots)$ function computes the previous decomposition with the geometric means of all four scale effects and all four output mix effects. The corresponding individual terms associated to either 'Path A' and 'Path B', or 'Path C' and 'Path D', can be recovered by using the previous (base) or (comparison) syntax, respectively. It is then possible to confirm that both decompositions are generally different and their geometric mean is a useful compromise.

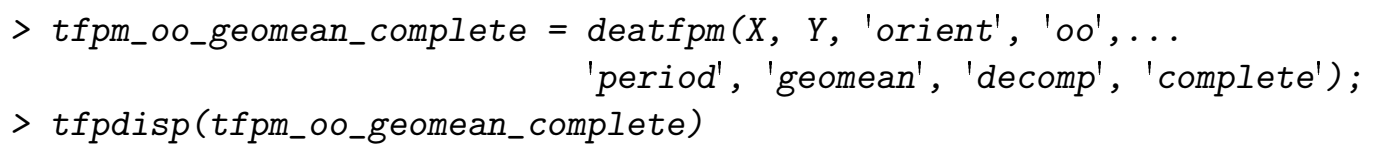

Total Factor Productivity (TFP)

DMUs : 5

Inputs: 2 Outputs: 2

Model: tfp-m

Orientation: oo (Output orientated)

Returns to scale: vrs (Variable)

\begin{tabular}{|c|c|c|c|c|c|}
\hline DMUI & $\mathrm{Ml}$ & $\mathrm{ECl}$ & $\mathrm{TCl}$ & $\mathrm{SEC} \mid$ & OME I \\
\hline 11 & 0.52041 & 0.58331 & 1.1055 & $0.8097 \mid$ & 0.9965 \\
\hline 21 & 1.2806 & 1.00001 & 1.3416 & 0.95601 & 0.9985 \\
\hline 31 & 1.00001 & 1.00001 & 1.00001 & 1.00001 & 1.0000 \\
\hline 41 & 2.50001 & 1.2500 & 1.00001 & 2.00001 & 1.0000 \\
\hline 51 & $0.5657 \mid$ & 0.60001 & $1.0000 \mid$ & $0.9428 \mid$ & 1.0000 \\
\hline
\end{tabular}

Malmquist:

Reference period is Geometric mean

$M=$ Malmquist. $E C=$ Efficiency Change. $T C=$ Technological Change.

SEC = Scale Effect. OME = Output Mix Effect.

\subsection{The input orientated MPI}


As a cone technology exhibits constant returns to scale, $\check{D}_{o}^{t}\left(\boldsymbol{x}^{t}, \boldsymbol{y}^{t}\right)=1 / \check{D}_{i}^{t}\left(\boldsymbol{x}^{t}, \boldsymbol{y}^{t}\right)$, and thus the productivity index defined by expression (1) can also be written as

$$
\check{M}_{o}^{t}\left(\boldsymbol{x}^{1}, \boldsymbol{y}^{1}, \boldsymbol{x}^{0}, \boldsymbol{y}^{0}\right)=\frac{\check{D}_{i}^{t}\left(\boldsymbol{x}^{0}, \boldsymbol{y}^{0}\right)}{\check{D}_{i}^{t}\left(\boldsymbol{x}^{1}, \boldsymbol{y}^{1}\right)} \equiv \check{M}_{i}^{t}\left(\boldsymbol{x}^{1}, \boldsymbol{y}^{1}, \boldsymbol{x}^{0}, \boldsymbol{y}^{0}\right)
$$

which defines the input orientated MPI, conditional on the period $t$ cone technology. The options for decomposing an input orientated MPI run parallel to those already presented.

The input orientated, base period MPI

For the base period viewpoint the four-factor decompositions are

$$
\begin{aligned}
\check{M}_{i}^{0}\left(\boldsymbol{x}^{1}, \boldsymbol{y}^{1}, \boldsymbol{x}^{0}, \boldsymbol{y}^{0}\right)= & E C_{i}\left(\boldsymbol{x}^{1}, \boldsymbol{y}^{1}, \boldsymbol{x}^{0}, \boldsymbol{y}^{0}\right) \times T C_{i}^{1,0}\left(\boldsymbol{x}^{1}, \boldsymbol{y}^{1}\right) \times \\
& I M E^{0}\left(\boldsymbol{x}^{1}, \boldsymbol{x}^{0}, \boldsymbol{y}^{0}\right) \times S E C_{i}^{0}\left(\boldsymbol{x}^{1}, \boldsymbol{y}^{1}, \boldsymbol{y}^{0}\right),
\end{aligned}
$$

and

$$
\begin{aligned}
\check{M}_{i}^{0}\left(\boldsymbol{x}^{1}, \boldsymbol{y}^{1}, \boldsymbol{x}^{0}, \boldsymbol{y}^{0}\right)= & E C_{i}\left(\boldsymbol{x}^{1}, \boldsymbol{y}^{1}, \boldsymbol{x}^{0}, \boldsymbol{y}^{0}\right) \times T C_{i}^{1,0}\left(\boldsymbol{x}^{1}, \boldsymbol{y}^{1}\right) \times \\
& I M E^{0}\left(\boldsymbol{x}^{1}, \boldsymbol{x}^{0}, \boldsymbol{y}^{1}\right) \times S E C_{i}^{0}\left(\boldsymbol{x}^{0}, \boldsymbol{y}^{1}, \boldsymbol{y}^{0}\right),
\end{aligned}
$$

identified in (Balk and Zofío 2018, Figure 2) by 'Path E' and 'Path F', respectively. Again, these two equally meaningful decompositions of the MPI index, differing with respect to the factor measuring the input mix effect and the factor jointly measuring the radial scale and output mix effect, can be consolidated by taking the geometric mean of expressions (17) and $(18)$

$$
\begin{aligned}
\check{M}_{i}^{0}\left(\boldsymbol{x}^{1}, \boldsymbol{y}^{1}, \boldsymbol{x}^{0}, \boldsymbol{y}^{0}\right)= & M_{i}^{0}\left(\boldsymbol{x}^{1}, \boldsymbol{y}^{1}, \boldsymbol{x}^{0}, \boldsymbol{y}^{0}\right) \times \\
& {\left[I M E^{0}\left(\boldsymbol{x}^{1}, \boldsymbol{x}^{0}, \boldsymbol{y}^{0}\right) \times I M E^{0}\left(\boldsymbol{x}^{1}, \boldsymbol{x}^{0}, \boldsymbol{y}^{1}\right)\right]^{1 / 2} } \\
& {\left[S E C_{i}^{0}\left(\boldsymbol{x}^{0}, \boldsymbol{y}^{1}, \boldsymbol{y}^{0}\right) \times S E C_{i}^{0}\left(\boldsymbol{x}^{1}, \boldsymbol{y}^{1}, \boldsymbol{y}^{0}\right)\right]^{1 / 2} . }
\end{aligned}
$$

To compute the input orientated, base period MPI in MATLAB we call the deatfpm(X, Y, ... ) function with: i) the orient parameter set to the input orientation (io); 2) the period parameter set to (base); and iii) the decomposition parameter decomp set to (complete).

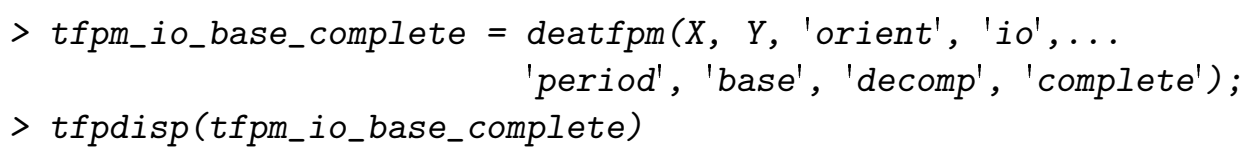

Total Factor Productivity (TFP)

DMUs : 5

Inputs: 2 Outputs: 2

Model: tfp-m

Orientation: io (Input orientated)

Returns to scale: vrs (Variable) 


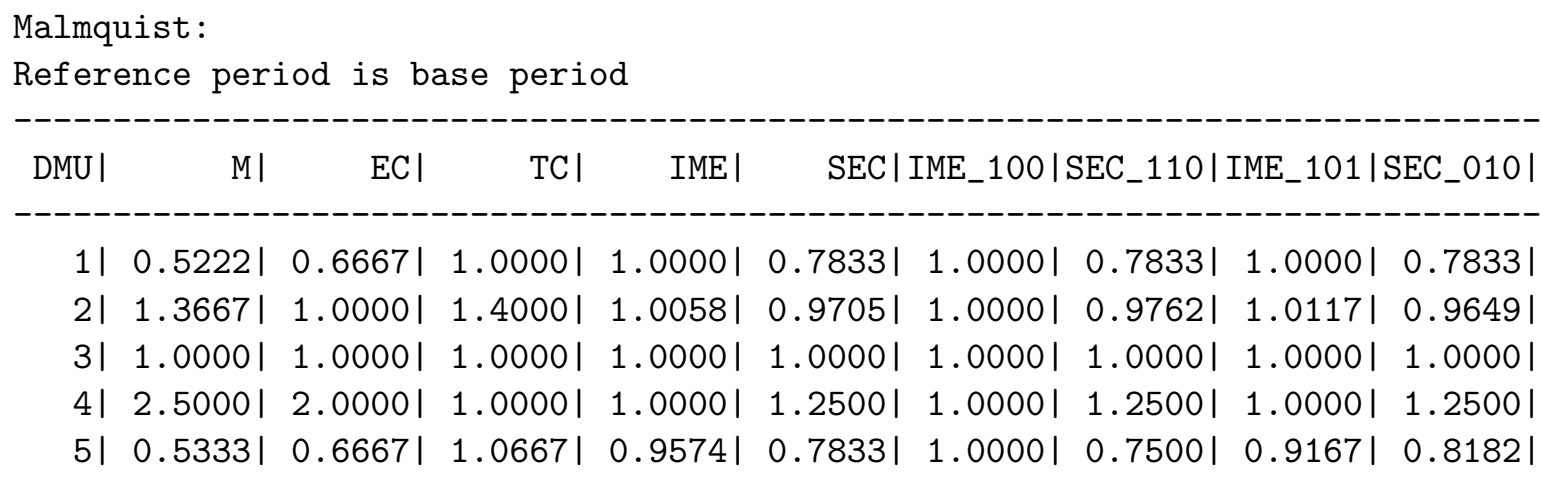

$M=$ Malmquist. $E C=$ Efficiency Change. $T C=$ Technological Change.

IME $=$ Input Mix Effect. SEC = Scale Effect.

Now the output of the function successively shows the MPI (M), which is equal to its base period, output orientated counterpart, the technical efficiency change factor (EC), the technological change factor (TC), the geometric mean of the input mix effect factors (IME), and that of the scale and output mix effect factors (SEC). The last four columns contain the values of these last two factors corresponding to 'Path E' and 'Path F' above, identified by the time superscripts of each of the input and output arguments; e.g., IME_100 corresponds to $I M E_{o}^{0}\left(\boldsymbol{x}^{1}, \boldsymbol{x}^{0}, \boldsymbol{y}^{0}\right)$ in expressions (17) and (19).

Related decompositions of the input orientated, base period MPI

Merging the input mix effects and scale effects in expressions (17) or (18) into the returns to scale factor proposed by Ray and Desli (1997) yields the three-factor decomposition

$$
\check{M}_{i}^{0}\left(\boldsymbol{x}^{1}, \boldsymbol{y}^{1}, \boldsymbol{x}^{0}, \boldsymbol{y}^{0}\right)=E C_{i}\left(\boldsymbol{x}^{1}, \boldsymbol{y}^{1}, \boldsymbol{x}^{0}, \boldsymbol{y}^{0}\right) \times T C_{i}^{1,0}\left(\boldsymbol{x}^{1}, \boldsymbol{y}^{1}\right) \times \frac{I S E^{0}\left(\boldsymbol{x}^{1}, \boldsymbol{y}^{1}\right)}{I S E^{0}\left(\boldsymbol{x}^{0}, \boldsymbol{y}^{0}\right)},
$$

where $I S E^{0}\left(\boldsymbol{x}^{1}, \boldsymbol{y}^{1}\right) / I S E^{0}\left(\boldsymbol{x}^{0}, \boldsymbol{y}^{0}\right)=\left[D_{i}^{0}\left(\boldsymbol{x}^{1}, \boldsymbol{y}^{1}\right) / \check{D}_{i}^{0}\left(\boldsymbol{x}^{1}, \boldsymbol{y}^{1}\right)\right] \times\left[\check{D}_{i}^{0}\left(\boldsymbol{x}^{0}, \boldsymbol{y}^{0}\right) / D_{i}^{0}\left(\boldsymbol{x}^{0}, \boldsymbol{y}^{0}\right)\right]$. To obtain this simpler decomposition the syntax is the same as the one above but the parameter decomp changed to (rts). The column headed RTS provides the contribution of returns to scale.

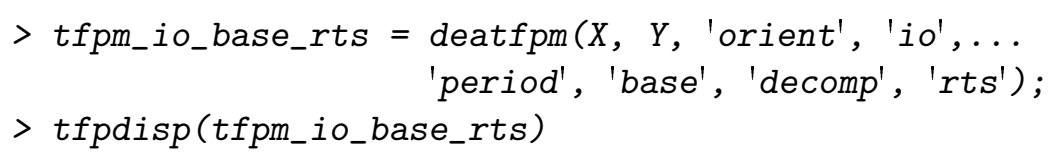

Total Factor Productivity (TFP)

DMUs : 5

Inputs: 2 Outputs: 2

Model: tfp-m

Orientation: io (Input orientated) 


\begin{tabular}{|c|c|c|c|c|}
\hline \multicolumn{5}{|c|}{$\begin{array}{l}\text { Malmquist: } \\
\text { Reference period is base period }\end{array}$} \\
\hline DMUI & $\mathrm{MI}$ & $\mathrm{ECl}$ & $\mathrm{TCl}$ & RTS I \\
\hline 11 & 0.52221 & $0.6667 \mid$ & $1.0000 \mid$ & 0.7833 \\
\hline 21 & $1.3667 \mid$ & $1.0000 \mid$ & 1.40001 & 0.9762 \\
\hline 31 & 1.00001 & $1.0000 \mid$ & 1.00001 & 1.00001 \\
\hline 41 & 2.50001 & 2.00001 & 1.00001 & 1.2500 \\
\hline 51 & $0.5333 \mid$ & $0.6667 \mid$ & $1.0667 \mid$ & 0.75001 \\
\hline
\end{tabular}

$M=$ Malmquist. $\mathrm{EC}=$ Efficiency Change. $\mathrm{TC}=$ Technological Change.

RTS $=$ Returns to Scale.

As in the output orientated case, the technical efficiency change and technological change factors common to expressions (17) and (18) correspond to the base period, input orientated version of the $C C D$ index,

$$
M_{i}^{0}\left(\boldsymbol{x}^{1}, \boldsymbol{y}^{1}, \boldsymbol{x}^{0}, \boldsymbol{y}^{0}\right) \equiv E C_{i}\left(\boldsymbol{x}^{1}, \boldsymbol{y}^{1}, \boldsymbol{x}^{0}, \boldsymbol{y}^{0}\right) \times T C_{i}^{1,0}\left(\boldsymbol{x}^{1}, \boldsymbol{y}^{1}\right)=\frac{D_{i}^{0}\left(\boldsymbol{x}^{0}, \boldsymbol{y}^{0}\right)}{D_{i}^{0}\left(\boldsymbol{x}^{1}, \boldsymbol{y}^{1}\right)}
$$

Therefore, substituting this in any of the above decompositions yields alternative expressions. For instance, for expressions (17) and (18) we obtain

$$
\check{M}_{i}^{0}\left(\boldsymbol{x}^{1}, \boldsymbol{y}^{1}, \boldsymbol{x}^{0}, \boldsymbol{y}^{0}\right)=M_{i}^{0}\left(\boldsymbol{x}^{1}, \boldsymbol{y}^{1}, \boldsymbol{x}^{0}, \boldsymbol{y}^{0}\right) \times I M E^{0}\left(\boldsymbol{x}^{1}, \boldsymbol{x}^{0}, \boldsymbol{y}^{0}\right) \times S E C_{i}^{0}\left(\boldsymbol{x}^{1}, \boldsymbol{y}^{1}, \boldsymbol{y}^{0}\right),
$$

and

$$
\check{M}_{i}^{0}\left(\boldsymbol{x}^{1}, \boldsymbol{y}^{1}, \boldsymbol{x}^{0}, \boldsymbol{y}^{0}\right)=M_{i}^{0}\left(\boldsymbol{x}^{1}, \boldsymbol{y}^{1}, \boldsymbol{x}^{0}, \boldsymbol{y}^{0}\right) \times I M E^{0}\left(\boldsymbol{x}^{1}, \boldsymbol{x}^{0}, \boldsymbol{y}^{1}\right) \times S E C_{i}^{0}\left(\boldsymbol{x}^{0}, \boldsymbol{y}^{1}, \boldsymbol{y}^{0}\right),
$$

respectively. The decomposition showing the $C C D$ index rather than its individual components relies on the same deatfpm (X, Y, ...) function under input orientation (io) and base period (base), but the parameter decomp must be changed from (complete) to (ccd). Notice that the $C C D$ index does not satisfy the proportionality requirement. Therefore the two-factor $C C D$ index decomposition is only sensible if, after performing the test for constant returns to scale by using the function deatestrtsm (X, Y, ...), with the orient parameter now set to (io), the null hypotheses of CRS cannot be rejected. Then the CRS version of the $C C D$ index can be calculated by setting the parameter decomp to (crs), i.e.,

$$
\check{M}_{i}^{0}\left(\boldsymbol{x}^{1}, \boldsymbol{y}^{1}, \boldsymbol{x}^{0}, \boldsymbol{y}^{0}\right)=\check{E C_{i}}\left(\boldsymbol{x}^{1}, \boldsymbol{y}^{1}, \boldsymbol{x}^{0}, \boldsymbol{y}^{0}\right) \times \check{T C_{i}^{1,0}}\left(\boldsymbol{x}^{1}, \boldsymbol{y}^{1}\right)
$$

whose output can be interpreted as in expression (9). 


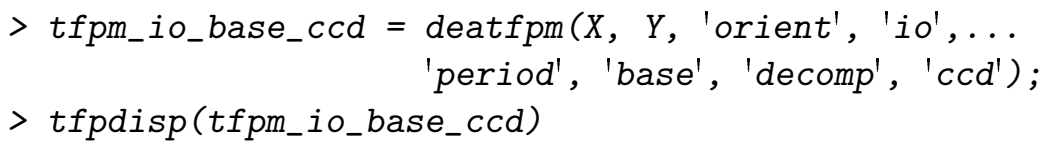

Total Factor Productivity (TFP)

DMUs : 5

Inputs: 2 Outputs: 2

Model: tfp-m

Orientation: io (Input orientated)

Returns to scale: vrs (Variable)

Malmquist :

Reference period is base period

\begin{tabular}{|c|c|c|c|c|c|c|c|c|}
\hline DMU I & $\mathrm{Ml}$ & Mvrs | & IME I & SEC I & IME_100| & SEC_110I & IME_101| & SEC_010I \\
\hline 1 & & & & & & & & \\
\hline 21 & 1.3667 & 1.4000 & $1.0058 \mid$ & 0.9705 & 1.00001 & $0.9762 \mid$ & 1.0117 & 0.964 \\
\hline 31 & 1.00001 & 1.00001 & 1.00001 & 1.0000 & 1.00001 & 1.00001 & 1.00001 & 1.000 \\
\hline 41 & 2.50001 & 2.00001 & $1.0000 \mid$ & 1.2500 & $1.0000 \mid$ & $1.2500 \mid$ & 1.00001 & 1.250 \\
\hline 51 & $0.5333 \mid$ & $0.7111 \mid$ & 0.95741 & 0.78331 & $1.0000 \mid$ & 0.75001 & $0.9167 \mid$ & 0.818 \\
\hline
\end{tabular}

$M=$ Malmquist. Mvrs = Malmquist VRS. IME = Input Mix Effect.

SEC = Scale Effect.

The input orientated, comparison period MPI

Choosing the comparison period cone technology, $\check{S}^{1}$, as benchmark yields the input orientated, comparison period MPI, $\check{M}_{i}^{1}\left(\boldsymbol{x}^{1}, \boldsymbol{y}^{1}, \boldsymbol{x}^{0}, \boldsymbol{y}^{0}\right)$, with the four-factor decompositions

$$
\begin{aligned}
\check{M}_{i}^{1}\left(\boldsymbol{x}^{1}, \boldsymbol{y}^{1}, \boldsymbol{x}^{0}, \boldsymbol{y}^{0}\right)= & E C_{i}\left(\boldsymbol{x}^{1}, \boldsymbol{y}^{1}, \boldsymbol{x}^{0}, \boldsymbol{y}^{0}\right) \times T C_{i}^{1,0}\left(\boldsymbol{x}^{0}, \boldsymbol{y}^{0}\right) \times \\
& I M E^{1}\left(\boldsymbol{x}^{1}, \boldsymbol{x}^{0}, \boldsymbol{y}^{0}\right) \times S E C_{i}^{1}\left(\boldsymbol{x}^{1}, \boldsymbol{y}^{1}, \boldsymbol{y}^{0}\right),
\end{aligned}
$$

and

$$
\begin{aligned}
\check{M}_{i}^{1}\left(\boldsymbol{x}^{1}, \boldsymbol{y}^{1}, \boldsymbol{x}^{0}, \boldsymbol{y}^{0}\right)= & E C_{i}\left(\boldsymbol{x}^{1}, \boldsymbol{y}^{1}, \boldsymbol{x}^{0}, \boldsymbol{y}^{0}\right) \times T C_{i}^{1,0}\left(\boldsymbol{x}^{0}, \boldsymbol{y}^{0}\right) \times \\
& I M E^{1}\left(\boldsymbol{x}^{1}, \boldsymbol{x}^{0}, \boldsymbol{y}^{1}\right) \times S E C_{i}^{1}\left(\boldsymbol{x}^{0}, \boldsymbol{y}^{1}, \boldsymbol{y}^{0}\right),
\end{aligned}
$$

corresponding to 'Path G' and 'Path H' in Balk and Zofío (2018). Following previous cases, these two decompositions can be aggregated by taking their geometric mean,

$$
\begin{aligned}
\check{M}_{i}^{1}\left(\boldsymbol{x}^{1}, \boldsymbol{y}^{1}, \boldsymbol{x}^{0}, \boldsymbol{y}^{0}\right)= & M_{i}^{1}\left(\boldsymbol{x}^{1}, \boldsymbol{y}^{1}, \boldsymbol{x}^{0}, \boldsymbol{y}^{0}\right) \times \\
& {\left[I M E^{1}\left(\boldsymbol{x}^{1}, \boldsymbol{x}^{0}, \boldsymbol{y}^{0}\right) \times I M E^{1}\left(\boldsymbol{x}^{1}, \boldsymbol{x}^{0}, \boldsymbol{y}^{1}\right)\right]^{1 / 2} } \\
& {\left[S E C_{i}^{1}\left(\boldsymbol{x}^{0}, \boldsymbol{y}^{1}, \boldsymbol{y}^{0}\right) \times S E C_{i}^{1}\left(\boldsymbol{x}^{1}, \boldsymbol{y}^{1}, \boldsymbol{y}^{0}\right)\right]^{1 / 2} . }
\end{aligned}
$$


Calculating the complete input orientated, comparison period MPI only requires changing (base) to (comparison) in the period parameter of the deatfpm $(\mathrm{X}, \mathrm{Y}, \ldots)$ function.

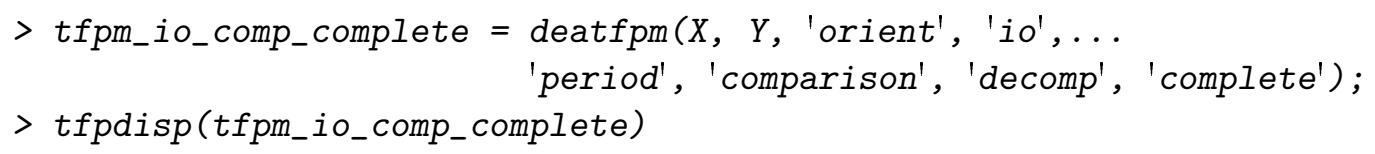

Total Factor Productivity (TFP)

DMUs : 5

Inputs: 2 Outputs: 2

Model: tfp-m

Orientation: io (Input orientated)

Returns to scale: vrs (Variable)

Malmquist:

Reference period is comparison period

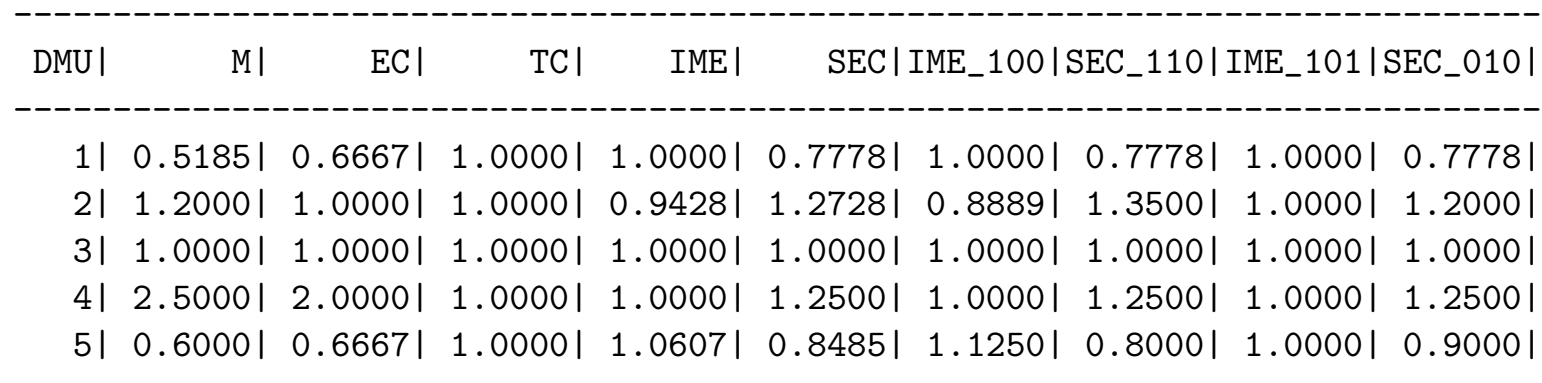

$\mathrm{M}=$ Malmquist. $\mathrm{EC}=$ Efficiency Change. $\mathrm{TC}=$ Technological Change.

IME $=$ Input Mix Effect. SEC = Scale Effect.

Related decompositions can easily be obtained by setting the parameter decomp to either (rts), (ccd), or (crs). For each one of these variants, the output of the function is interpreted in the same way as the base period counterparts.

The input orientated, geometric mean MPI

The base and comparison period perspectives yield generally different results. To prevent an arbitrary choice of reference technology, the geometric mean of the base and comparison indices is defined by

$$
\begin{gathered}
\check{M}_{i}\left(\boldsymbol{x}^{1}, \boldsymbol{y}^{1}, \boldsymbol{x}^{0}, \boldsymbol{y}^{0}\right) \equiv\left[\check{M}_{i}^{0}\left(\boldsymbol{x}^{1}, \boldsymbol{y}^{1}, \boldsymbol{x}^{0}, \boldsymbol{y}^{0}\right) \times \check{M}_{i}^{1}\left(\boldsymbol{x}^{1}, \boldsymbol{y}^{1}, \boldsymbol{x}^{0}, \boldsymbol{y}^{0}\right)\right]^{1 / 2}= \\
{\left[\frac{\check{D}_{i}^{0}\left(\boldsymbol{x}^{0}, \boldsymbol{y}^{0}\right)}{\check{D}_{i}^{0}\left(\boldsymbol{x}^{1}, \boldsymbol{y}^{1}\right)} \frac{\check{D}_{i}^{1}\left(\boldsymbol{x}^{0}, \boldsymbol{y}^{0}\right)}{\check{D}_{i}^{1}\left(\boldsymbol{x}^{1}, \boldsymbol{y}^{1}\right)}\right]^{1 / 2} .}
\end{gathered}
$$

This index can be decomposed in the following ways,

$$
\check{M}_{i}\left(\boldsymbol{x}^{1}, \boldsymbol{y}^{1}, \boldsymbol{x}^{0}, \boldsymbol{y}^{0}\right)=
$$




$$
\begin{gathered}
{\left[M_{i}^{0}\left(\boldsymbol{x}^{1}, \boldsymbol{y}^{1}, \boldsymbol{x}^{0}, \boldsymbol{y}^{0}\right) M_{i}^{1}\left(\boldsymbol{x}^{1}, \boldsymbol{y}^{1}, \boldsymbol{x}^{0}, \boldsymbol{y}^{0}\right)\right]^{1 / 2} \times} \\
{\left[I M E^{0}\left(\boldsymbol{x}^{1}, \boldsymbol{x}^{0}, \boldsymbol{y}^{0}\right) I M E^{0}\left(\boldsymbol{x}^{1}, \boldsymbol{x}^{0}, \boldsymbol{y}^{1}\right) I M E^{1}\left(\boldsymbol{x}^{1}, \boldsymbol{x}^{0}, \boldsymbol{y}^{0}\right) I M E^{1}\left(\boldsymbol{x}^{1}, \boldsymbol{x}^{0}, \boldsymbol{y}^{1}\right)\right]^{1 / 4} \times} \\
{\left[S E C_{i}^{0}\left(\boldsymbol{x}^{0}, \boldsymbol{y}^{1}, \boldsymbol{y}^{0}\right) S E C_{i}^{0}\left(\boldsymbol{x}^{1}, \boldsymbol{y}^{1}, \boldsymbol{y}^{0}\right) S E C_{i}^{1}\left(\boldsymbol{x}^{0}, \boldsymbol{y}^{1}, \boldsymbol{y}^{0}\right) S E C_{i}^{1}\left(\boldsymbol{x}^{1}, \boldsymbol{y}^{1}, \boldsymbol{y}^{0}\right)\right]^{1 / 4}=} \\
{\left[M_{i}^{0}\left(\boldsymbol{x}^{1}, \boldsymbol{y}^{1}, \boldsymbol{x}^{0}, \boldsymbol{y}^{0}\right) M_{i}^{1}\left(\boldsymbol{x}^{1}, \boldsymbol{y}^{1}, \boldsymbol{x}^{0}, \boldsymbol{y}^{0}\right)\right]^{1 / 2} \times} \\
{\left[\left[I M E^{0}\left(\boldsymbol{x}^{1}, \boldsymbol{x}^{0}, \boldsymbol{y}^{0}\right) I M E^{1}\left(\boldsymbol{x}^{1}, \boldsymbol{x}^{0}, \boldsymbol{y}^{0}\right)\right]^{1 / 2}\left[I M E^{0}\left(\boldsymbol{x}^{1}, \boldsymbol{x}^{0}, \boldsymbol{y}^{1}\right) I M E^{1}\left(\boldsymbol{x}^{1}, \boldsymbol{x}^{0}, \boldsymbol{y}^{1}\right)\right]^{1 / 2}\right]^{1 / 2} \times} \\
{\left[\left[S E C_{i}^{0}\left(\boldsymbol{x}^{0}, \boldsymbol{y}^{1}, \boldsymbol{y}^{0}\right) S E C_{i}^{1}\left(\boldsymbol{x}^{0}, \boldsymbol{y}^{1}, \boldsymbol{y}^{0}\right)\right]^{1 / 2}\left[S E C_{i}^{0}\left(\boldsymbol{x}^{1}, \boldsymbol{y}^{1}, \boldsymbol{y}^{0}\right) S E C_{i}^{1}\left(\boldsymbol{x}^{1}, \boldsymbol{y}^{1}, \boldsymbol{y}^{0}\right)\right]^{1 / 2}\right]^{1 / 2}}
\end{gathered}
$$

where the second part after the last equality sign simply collects the input mix effects and scale effects with the same argument structure. This second decomposition shows what happens if we first combine decompositions along 'Path E' and 'Path G', and then along 'Path F' and 'Path H', and next combine these two terms.

Setting the period parameter to (geomean) in the deatfpm (X, Y, ...) function computes the previous decomposition with the geometric means of all four scale effects and all four output mix effects associated to the four different paths.

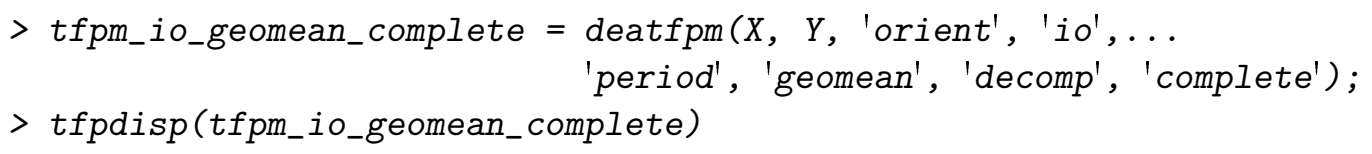

Total Factor Productivity (TFP)

DMUs : 5

Inputs: 2 Outputs: 2

\begin{tabular}{|c|c|c|c|c|c|}
\hline DMU I & MI & $\mathrm{ECl}$ & $\mathrm{TCl}$ & IME | & SECI \\
\hline 11 & $0.5204 \mid$ & $0.6667 \mid$ & 1.00001 & $1.0000 \mid$ & 0.7806 \\
\hline 21 & $1.2806 \mid$ & $1.0000 \mid$ & 1.1832 & 0.97381 & 1.1114 \\
\hline 31 & 1.00001 & $1.0000 \mid$ & 1.00001 & 1.00001 & 1.0000 \\
\hline 41 & 2.50001 & 2.00001 & 1.00001 & 1.00001 & 1.2500 \\
\hline 51 & $0.5657 \mid$ & $0.6667 \mid$ & $1.0328 \mid$ & $1.0077 \mid$ & 0.8153 \\
\hline
\end{tabular}

Model: tfp-m

Orientation: io (Input orientated)

Returns to scale: vrs (Variable)

Malmquist:

Reference period is Geometric mean

$M=$ Malmquist. $E C=$ Efficiency Change. $T C=$ Technological Change.

IME $=$ Input Mix Effect. SEC = Scale Effect. 


\section{Moorsteen-Bjurek Productivity Index}

The second definition of a productivity index based only on quantities takes into account both the output and input orientations. Specifically, the family of Moorsteen-Bjurek productivity indices (MBPI), is defined as the ratio of an output quantity index to an input quantity index. A Malmquist output quantity index, comparing output quantities $\boldsymbol{y}^{1}$ to $\boldsymbol{y}^{0}$, conditional on certain input quantities $\overline{\boldsymbol{x}}$, is defined as $Q_{o}^{t}\left(\boldsymbol{y}^{1}, \boldsymbol{y}^{0}, \overline{\boldsymbol{x}}\right) \equiv D_{o}^{t}\left(\overline{\boldsymbol{x}}, \boldsymbol{y}^{1}\right) / D_{o}^{t}\left(\overline{\boldsymbol{x}}, \boldsymbol{y}^{0}\right)$. Similarly, a Malmquist input quantity index, comparing input quantities $\boldsymbol{x}^{1}$ to $\boldsymbol{x}^{0}$, conditional on certain output quantities $\overline{\boldsymbol{y}}$, is defined as $Q_{i}^{t}\left(\boldsymbol{x}^{1}, \boldsymbol{x}^{0}, \overline{\boldsymbol{y}}\right) \equiv D_{i}^{t}\left(\boldsymbol{x}^{1}, \overline{\boldsymbol{y}}\right) / D_{i}^{t}\left(\boldsymbol{x}^{0}, \overline{\boldsymbol{y}}\right)$. Both indices can be traced back to suggestions by Moorsteen (1961), and their properties were extensively discussed in (Balk 1998, Sections 3.4 and 4.3). Typically, in empirical applications involving many observations, $\overline{\boldsymbol{x}}$ and $\overline{\boldsymbol{y}}$ would be chosen as vectors of sample means. This is the approach followed in the toolbox. The MBPI is then defined by

$$
M B^{t}\left(\boldsymbol{x}^{1}, \boldsymbol{y}^{1}, \boldsymbol{x}^{0}, \boldsymbol{y}^{0} ; \overline{\boldsymbol{x}}, \overline{\boldsymbol{y}}\right) \equiv \frac{Q_{o}^{t}\left(\boldsymbol{y}^{1}, \boldsymbol{y}^{0}, \overline{\boldsymbol{x}}\right)}{Q_{i}^{t}\left(\boldsymbol{x}^{1}, \boldsymbol{x}^{0}, \overline{\boldsymbol{y}}\right)}=\frac{D_{o}^{t}\left(\overline{\boldsymbol{x}}, \boldsymbol{y}^{1}\right) / D_{o}^{t}\left(\overline{\boldsymbol{x}}, \boldsymbol{y}^{0}\right)}{D_{i}^{t}\left(\boldsymbol{x}^{1}, \overline{\boldsymbol{y}}\right) / D_{i}^{t}\left(\boldsymbol{x}^{0}, \overline{\boldsymbol{y}}\right)}=\frac{D_{o}^{t}\left(\overline{\boldsymbol{x}}, \boldsymbol{y}^{1}\right) / D_{i}^{t}\left(\boldsymbol{x}^{1}, \overline{\boldsymbol{y}}\right)}{D_{o}^{t}\left(\overline{\boldsymbol{x}}, \boldsymbol{y}^{0}\right) / D_{i}^{t}\left(\boldsymbol{x}^{0}, \overline{\boldsymbol{y}}\right)}
$$

where the latter term is a ratio of two productivity levels. Up to a scalar normalization, and conditional on $\overline{\boldsymbol{x}}$ and $\overline{\boldsymbol{y}}$, the productivity level at the input-output situation $(\boldsymbol{x}, \boldsymbol{y})$ is thereby measured as $D_{o}^{t}(\overline{\boldsymbol{x}}, \boldsymbol{y}) / D_{i}^{t}(\boldsymbol{x}, \overline{\boldsymbol{y}})$, where superscript $t$ refers to the benchmark technology $S^{t}$. Thus, the MBPI belongs to the class of "multiplicatively complete" TFP indices, as defined by O'Donnell (2012). ${ }^{5}$

Based on the properties of the MBPI, Balk and Zofío (2018) show that this index can be decomposed into factors corresponding to those already shown and interpreted. Decompositions can be based on output distance functions or input distance functions. As benchmark the technologies of period 0 and 1 will be used. We follow the order in which the MPI decompositions were discussed.

\subsection{The output orientated decomposition of MBPI}

The output orientated decomposition of the base period MBPI

Taking as benchmark the base period technology, along 'Path A' the MBPI is decomposed as

$$
\begin{aligned}
M B^{0}\left(\boldsymbol{x}^{1}, \boldsymbol{y}^{1}, \boldsymbol{x}^{0}, \boldsymbol{y}^{0} ; \overline{\boldsymbol{x}}, \overline{\boldsymbol{y}}\right)= & E C_{o}\left(\boldsymbol{x}^{1}, \boldsymbol{y}^{1}, \boldsymbol{x}^{0}, \boldsymbol{y}^{0}\right) \times T C_{o}^{1,0}\left(\boldsymbol{x}^{1}, \boldsymbol{y}^{1}\right) \times \\
& \left(\frac{D_{o}^{0}\left(\boldsymbol{x}^{0}, \boldsymbol{y}^{0}\right)}{D_{o}^{0}\left(\boldsymbol{x}^{1}, \boldsymbol{y}^{0}\right)} \frac{D_{i}^{0}\left(\boldsymbol{x}^{0}, \overline{\boldsymbol{y}}\right)}{D_{i}^{0}\left(\boldsymbol{x}^{1}, \overline{\boldsymbol{y}}\right)}\right) \times\left(\frac{D_{o}^{0}\left(\overline{\boldsymbol{x}}, \boldsymbol{y}^{1}\right)}{D_{o}^{0}\left(\overline{\boldsymbol{x}}, \boldsymbol{y}^{0}\right)} \frac{D_{o}^{0}\left(\boldsymbol{x}^{1}, \boldsymbol{y}^{0}\right)}{D_{o}^{0}\left(\boldsymbol{x}^{1}, \boldsymbol{y}^{1}\right)}\right),
\end{aligned}
$$

where the factors on the right-hand side of the equality sign represent, respectively, technical efficiency change, technological change (conditional on $\left(\boldsymbol{x}^{1}, \boldsymbol{y}^{1}\right)$ ), the radial scale and input mix effect (conditional on $\boldsymbol{y}^{0}$ ), and the output mix effect (conditional on $\boldsymbol{x}^{1}$ ).

\footnotetext{
${ }^{5}$ O'Donnell (2014) called the indices defined by expression (30) after Färe and Primont because the component output and input quantity indices are discussed in Färe and Primont (1995). The indices were called Bjurek productivity indices by Diewert and Fox (2017).
} 
With the same benchmark technology, along 'Path B' the following decomposition is obtained:

$$
\begin{aligned}
M B^{0}\left(\boldsymbol{x}^{1}, \boldsymbol{y}^{1}, \boldsymbol{x}^{0}, \boldsymbol{y}^{0} ; \overline{\boldsymbol{x}}, \overline{\boldsymbol{y}}\right)= & E C_{o}\left(\boldsymbol{x}^{1}, \boldsymbol{y}^{1}, \boldsymbol{x}^{0}, \boldsymbol{y}^{0}\right) \times T C_{o}^{1,0}\left(\boldsymbol{x}^{1}, \boldsymbol{y}^{1}\right) \times \\
& \left(\frac{D_{o}^{0}\left(\boldsymbol{x}^{0}, \boldsymbol{y}^{1}\right)}{D_{o}^{0}\left(\boldsymbol{x}^{1}, \boldsymbol{y}^{1}\right)} \frac{D_{i}^{0}\left(\boldsymbol{x}^{0}, \overline{\boldsymbol{y}}\right)}{D_{i}^{0}\left(\boldsymbol{x}^{1}, \overline{\boldsymbol{y}}\right)}\right) \times\left(\frac{D_{o}^{0}\left(\overline{\boldsymbol{x}}, \boldsymbol{y}^{1}\right)}{D_{o}^{0}\left(\overline{\boldsymbol{x}}, \boldsymbol{y}^{0}\right)} \frac{D_{o}^{0}\left(\boldsymbol{x}^{0}, \boldsymbol{y}^{0}\right)}{D_{o}^{0}\left(\boldsymbol{x}^{0}, \boldsymbol{y}^{1}\right)}\right) .
\end{aligned}
$$

The first two terms are the same but the radial scale and input mix effect are now conditional on $\boldsymbol{y}^{1}$, while the output mix effect is conditional on $\boldsymbol{x}^{0}$. Both decompositions of MBPI can be computed through the deatfpmb $(\mathrm{X}, \mathrm{Y}, \ldots)$ function with: i) the orient parameter set to output orientation (oo);2) the period parameter set to (base); and iii) the decomposition parameter decomp set to (complete). In this function, the average of the inputs $\overline{\boldsymbol{x}}$ and outputs $\overline{\boldsymbol{y}}$ in two consecutive periods 0 and 1 are automatically calculated from the arrays of $\mathrm{X}$ and $\mathrm{Y}$.

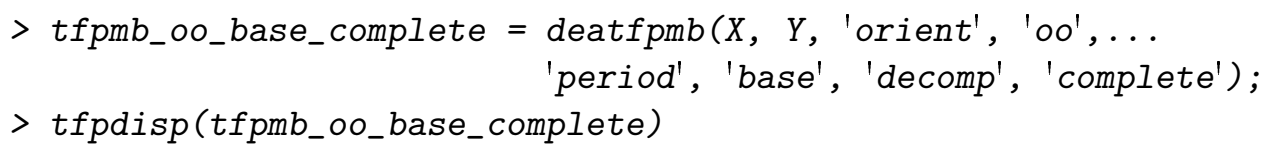

Total Factor Productivity (TFP)

DMUs : 5

Inputs: 2 Outputs: 2

Model: tfp-mb

Orientation: oo (Output orientated)

Returns to scale: vrs (Variable)

Moorsteen-Bjurek index:

Reference period is base period

DMUI MBI ECI TCI SECI OME ISEC_100IOME_110|SEC_101|OME_010|

\begin{tabular}{lllllllllll}
\hline $1 \mid$ & $0.5185 \mid$ & $0.5833 \mid$ & $1.0000 \mid$ & $0.9015 \mid$ & $0.9860 \mid$ & $0.8889 \mid$ & $1.0000 \mid$ & $0.9143 \mid$ & $0.9722 \mid$ \\
$2 \mid$ & $1.3385 \mid$ & $1.0000 \mid$ & $1.8000 \mid$ & $0.7504 \mid$ & $0.9909 \mid$ & $0.7436 \mid$ & $1.0000 \mid$ & $0.7574 \mid$ & $0.9818 \mid$ \\
$3 \mid$ & $1.0000 \mid$ & $1.0000 \mid$ & $1.0000 \mid$ & $1.0000 \mid$ & $1.0000 \mid$ & $1.0000 \mid$ & $1.0000 \mid$ & $1.0000 \mid$ & $1.0000 \mid$ \\
$4 \mid$ & $2.5000 \mid$ & $1.2500 \mid$ & $1.0000 \mid$ & $2.0000 \mid$ & $1.0000 \mid$ & $2.0000 \mid$ & $1.0000 \mid$ & $2.0000 \mid$ & $1.0000 \mid$ \\
$5 \mid$ & $0.5538 \mid$ & $0.6000 \mid$ & $1.0000 \mid$ & $0.9231 \mid$ & $1.0000 \mid$ & $0.9231 \mid$ & $1.0000 \mid$ & $0.9231 \mid$ & $1.0000 \mid$
\end{tabular}

$\mathrm{MB}=$ Moorsteen-Bjurek. $\mathrm{EC}=$ Efficiency Change. $\mathrm{TC}=$ Technological Change. SEC = Scale Effect. OME = Output Mix Effect.

The output of the function successively shows the MBPI (MB), the technical efficiency change factor (EC), the technological change factor (TC), the geometric mean of the scale effect factors (SEC), and that of the output mix effect factors (OME). The last four columns show the separate values of the last two factors corresponding to 'Path A' and 'Path B', identified by the time superscripts of each of the input and output arguments in each factor. For instance, 
the constant returns to scale version of the MBPI can be calculated by setting the parameter decomp to (crs). Expression (31) becomes

$$
\begin{aligned}
& M B^{0}\left(\boldsymbol{x}^{1}, \boldsymbol{y}^{1}, \boldsymbol{x}^{0}, \boldsymbol{y}^{0} ; \overline{\boldsymbol{x}}, \overline{\boldsymbol{y}}\right)=\check{E C_{o}}\left(\boldsymbol{x}^{1}, \boldsymbol{y}^{1}, \boldsymbol{x}^{0}, \boldsymbol{y}^{0}\right) \times \check{T C_{o}^{1,0}}\left(\boldsymbol{x}^{1}, \boldsymbol{y}^{1}\right) \times \\
& \left(\frac{\check{D}_{o}^{0}\left(\boldsymbol{x}^{0}, \boldsymbol{y}^{0}\right)}{\check{D}_{o}^{0}\left(\boldsymbol{x}^{1}, \boldsymbol{y}^{0}\right)} \frac{\check{D}_{i}^{0}\left(\boldsymbol{x}^{0}, \overline{\boldsymbol{y}}\right)}{\check{D}_{i}^{0}\left(\boldsymbol{x}^{1}, \overline{\boldsymbol{y}}\right)}\right) \times\left(\frac{\check{D}_{o}^{0}\left(\overline{\boldsymbol{x}}, \boldsymbol{y}^{1}\right)}{\check{D}_{o}^{0}\left(\overline{\boldsymbol{x}}, \boldsymbol{y}^{0}\right)} \frac{\check{D}_{o}^{0}\left(\boldsymbol{x}^{1}, \boldsymbol{y}^{0}\right)}{\check{D}_{o}^{0}\left(\boldsymbol{x}^{1}, \boldsymbol{y}^{1}\right)}\right),
\end{aligned}
$$

where the third factor measures the input mix effect, because the radial scale component vanishes, and the fourth factor measures the output mix effect.

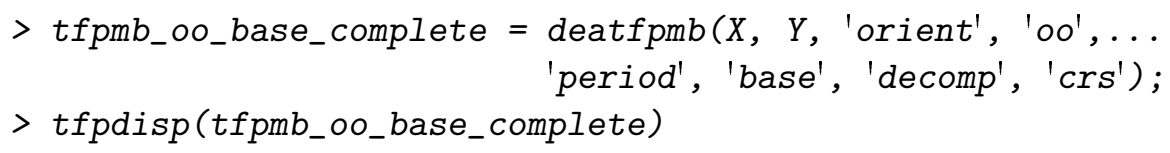

Total Factor Productivity (TFP)

DMUs : 5

Inputs: 2 Outputs: 2

Model: tfp-mb

Orientation: oo (Output orientated)

Returns to scale: crs (Constant)

Moorsteen-Bjurek index:

Reference period is base period

DMU I MBI $\quad$ ECI TCI SECI OME|SEC_100|OME_110|SEC_101|OME_010|

$1|0.5222| 0.4242|1.2310| 1.0000|1.0000| 1.0000|1.0000| 1.0000|1.0000|$

$2|1.3627| 1.0000|1.3667| 1.0001|0.9970| 0.9971|1.0000| 1.0032|0.9939|$

$3|1.0000| 0.8333|1.2000| 1.0000|1.0000| 1.0000|1.0000| 1.0000|1.0000|$

$4|2.5000| 2.0455|1.2222| 1.0000|1.0000| 1.0000|1.0000| 1.0000|1.0000|$

$5|0.5333| 0.4444|1.2000| 1.0000|1.0000| 1.0000|1.0000| 1.0000|1.0000|$

$M B=$ Moorsteen-Bjurek. $E C=$ Efficiency Change. $T C=$ Technological Change.

$\mathrm{SEC}=$ Scale Effect. OME = Output Mix Effect.

Under CRS the SEC factor measures only the input mix effect.

The output orientated decomposition of the comparion period MBPI

Similar decompositions of the MBPI can be obtained by taking the comparison period technology as benchmark. These concur with the decompositions corresponding to 'Path $\mathrm{C}$ ' and 
'Path D' of the output orientated MPI:

$$
\begin{aligned}
M B^{1}\left(\boldsymbol{x}^{1}, \boldsymbol{y}^{1}, \boldsymbol{x}^{0}, \boldsymbol{y}^{0} ; \overline{\boldsymbol{x}}, \overline{\boldsymbol{y}}\right)= & E C_{o}\left(\boldsymbol{x}^{1}, \boldsymbol{y}^{1}, \boldsymbol{x}^{0}, \boldsymbol{y}^{0}\right) \times T C_{o}^{1,0}\left(\boldsymbol{x}^{0}, \boldsymbol{y}^{0}\right) \times \\
& \left(\frac{D_{o}^{1}\left(\boldsymbol{x}^{0}, \boldsymbol{y}^{0}\right)}{D_{o}^{1}\left(\boldsymbol{x}^{1}, \boldsymbol{y}^{0}\right)} \frac{D_{i}^{1}\left(\boldsymbol{x}^{0}, \overline{\boldsymbol{y}}\right)}{D_{i}^{1}\left(\boldsymbol{x}^{1}, \overline{\boldsymbol{y}}\right)}\right) \times\left(\frac{D_{o}^{1}\left(\overline{\boldsymbol{x}}, \boldsymbol{y}^{1}\right)}{D_{o}^{1}\left(\overline{\boldsymbol{x}}, \boldsymbol{y}^{0}\right)} \frac{D_{o}^{1}\left(\boldsymbol{x}^{1}, \boldsymbol{y}^{0}\right)}{D_{o}^{1}\left(\boldsymbol{x}^{1}, \boldsymbol{y}^{1}\right)}\right),
\end{aligned}
$$

and

$$
\begin{aligned}
M B^{1}\left(\boldsymbol{x}^{1}, \boldsymbol{y}^{1}, \boldsymbol{x}^{0}, \boldsymbol{y}^{0} ; \overline{\boldsymbol{x}}, \overline{\boldsymbol{y}}\right)= & E C_{o}\left(\boldsymbol{x}^{1}, \boldsymbol{y}^{1}, \boldsymbol{x}^{0}, \boldsymbol{y}^{0}\right) \times T C_{o}^{1,0}\left(\boldsymbol{x}^{0}, \boldsymbol{y}^{0}\right) \times \\
& \left(\frac{D_{o}^{1}\left(\boldsymbol{x}^{0}, \boldsymbol{y}^{1}\right)}{D_{o}^{1}\left(\boldsymbol{x}^{1}, \boldsymbol{y}^{1}\right)} \frac{D_{i}^{1}\left(\boldsymbol{x}^{0}, \overline{\boldsymbol{y}}\right)}{D_{i}^{1}\left(\boldsymbol{x}^{1}, \overline{\boldsymbol{y}}\right)}\right) \times\left(\frac{D_{o}^{1}\left(\overline{\boldsymbol{x}}, \boldsymbol{y}^{1}\right)}{D_{o}^{1}\left(\overline{\boldsymbol{x}}, \boldsymbol{y}^{0}\right)} \frac{D_{o}^{1}\left(\boldsymbol{x}^{0}, \boldsymbol{y}^{0}\right)}{D_{o}^{1}\left(\boldsymbol{x}^{0}, \boldsymbol{y}^{1}\right)}\right) .
\end{aligned}
$$

To obtain these decompositions, in the deatfpmb $(\mathrm{X}, \mathrm{Y}, \ldots)$ function the period parameter must be set to (comparison). Again, if the user prefers related decompositions rather than the (complete) one, then the decomp parameter can be changed to (ccd) or (rts).

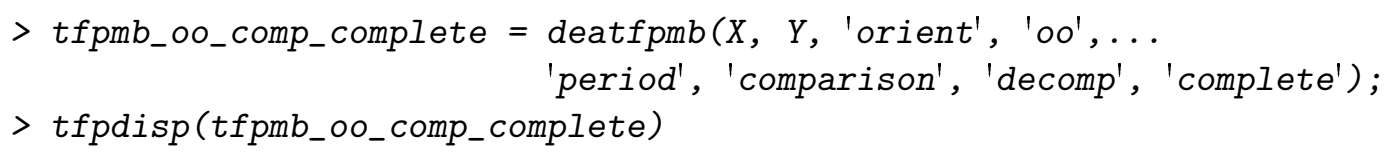

Total Factor Productivity (TFP)

DMUs : 5

Inputs: 2 Outputs: 2

Model: tfp-mb

Orientation: oo (Output orientated)

Returns to scale: vrs (Variable)

Moorsteen-Bjurek index:

Reference period is comparison period

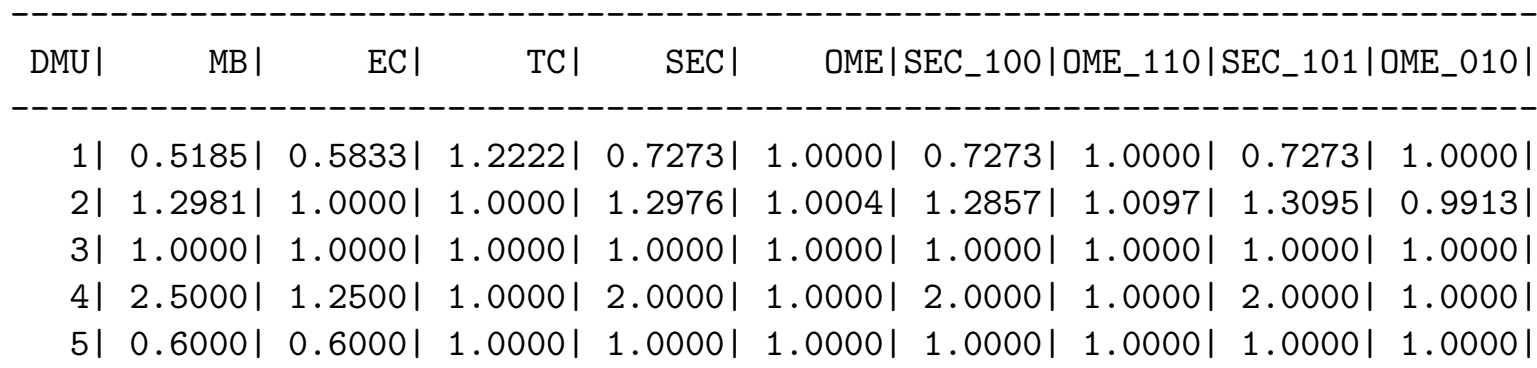

$M B=$ Moorsteen-Bjurek. $E C=$ Efficiency Change. $T C=$ Technological Change.

$\mathrm{SEC}=$ Scale Effect. OME = Output Mix Effect.

The output orientated decomposition of the geometric mean MBPI

The base and comparison period MBPIs yield different results unless the benchmark technologies satisfy extremely restrictive conditions. Thus we have four decompositions of the 
productivity index $M B^{t}\left(\boldsymbol{x}^{1}, \boldsymbol{y}^{1}, \boldsymbol{x}^{0}, \boldsymbol{y}^{0} ; \overline{\boldsymbol{x}}, \overline{\boldsymbol{y}}\right)$ from an output orientation. Expressions (31) and (32) provide two decompositions of $M B^{0}\left(\boldsymbol{x}^{1}, \boldsymbol{y}^{1}, \boldsymbol{x}^{0}, \boldsymbol{y}^{0} ; \overline{\boldsymbol{x}}, \overline{\boldsymbol{y}}\right)$. Similarly, expressions (35) and (36) provide two decompositions of $M B^{1}\left(\boldsymbol{x}^{1}, \boldsymbol{y}^{1}, \boldsymbol{x}^{0}, \boldsymbol{y}^{0} ; \overline{\boldsymbol{x}}, \overline{\boldsymbol{y}}\right)$. By taking their geometric mean, we obtain a decomposition of the geometric mean index $M B\left(\boldsymbol{x}^{1}, \boldsymbol{y}^{1}, \boldsymbol{x}^{0}\right.$, $\left.\boldsymbol{y}^{0} ; \overline{\boldsymbol{x}}, \overline{\boldsymbol{y}}\right)=\left[M B^{0}\left(\boldsymbol{x}^{1}, \boldsymbol{y}^{1}, \boldsymbol{x}^{0}, \boldsymbol{y}^{0} ; \overline{\boldsymbol{x}}, \overline{\boldsymbol{y}}\right) M B^{1}\left(\boldsymbol{x}^{1}, \boldsymbol{y}^{1}, \boldsymbol{x}^{0}, \boldsymbol{y}^{0} ; \overline{\boldsymbol{x}}, \overline{\boldsymbol{y}}\right)\right]^{1 / 2}$. Thus,

$$
\begin{aligned}
& M B\left(\boldsymbol{x}^{1}, \boldsymbol{y}^{1}, \boldsymbol{x}^{0}, \boldsymbol{y}^{0} ; \overline{\boldsymbol{x}}, \overline{\boldsymbol{y}}\right)=E C_{o}\left(\boldsymbol{x}^{1}, \boldsymbol{y}^{1}, \boldsymbol{x}^{0}, \boldsymbol{y}^{0}\right) \times\left[T C_{o}^{0,1}\left(\boldsymbol{x}^{1}, \boldsymbol{y}^{1}\right) \times T C_{o}^{0,1}\left(\boldsymbol{x}^{0}, \boldsymbol{y}^{0}\right)\right]^{1 / 2} \times \\
& \underbrace{[\underbrace{\left(\frac{D_{o}^{0}\left(\boldsymbol{x}^{0}, \boldsymbol{y}^{0}\right)}{D_{o}^{0}\left(\boldsymbol{x}^{1}, \boldsymbol{y}^{0}\right)} \frac{D_{i}^{0}\left(\boldsymbol{x}^{0}, \overline{\boldsymbol{y}}\right)}{D_{i}^{0}\left(\boldsymbol{x}^{1}, \overline{\boldsymbol{y}}\right)}\right)}_{\text {Third term Path A, SEC }} \times \underbrace{\left(\frac{D_{o}^{1}\left(\boldsymbol{x}^{0}, \boldsymbol{y}^{0}\right)}{D_{o}^{1}\left(\boldsymbol{x}^{1}, \boldsymbol{y}^{0}\right)} \frac{D_{i}^{1}\left(\boldsymbol{x}^{0}, \overline{\boldsymbol{y}}\right)}{D_{i}^{1}\left(\boldsymbol{x}^{1}, \overline{\boldsymbol{y}}\right)}\right)}_{\text {Third term Path } C, \text { SEC }}]^{1 / 2}}_{\text {Third terms paths AC, SEC }} \\
& \underbrace{[\underbrace{\left(\frac{D_{o}^{0}\left(\boldsymbol{x}^{0}, \boldsymbol{y}^{1}\right)}{D_{o}^{0}\left(\boldsymbol{x}^{1}, \boldsymbol{y}^{1}\right)} \frac{D_{i}^{0}\left(\boldsymbol{x}^{0}, \overline{\boldsymbol{y}}\right)}{D_{i}^{0}\left(\boldsymbol{x}^{1}, \overline{\boldsymbol{y}}\right)}\right)}_{\text {Third term Path B, SEC }} \times \underbrace{\left(\frac{D_{o}^{1}\left(\boldsymbol{x}^{0}, y^{1}\right)}{D_{o}^{1}\left(\boldsymbol{x}^{1}, \boldsymbol{y}^{1}\right)} \frac{D_{i}^{1}\left(\boldsymbol{x}^{0}, \overline{\boldsymbol{y}}\right)}{D_{i}^{1}\left(\boldsymbol{x}^{1}, \bar{y}\right)}\right)}_{\text {Third term Path D, SEC }}]^{1 / 2} \times}_{\text {Third terms paths BD, SEC }} \\
& \times \underbrace{[\underbrace{\left(\frac{D_{o}^{0}\left(\overline{\boldsymbol{x}}, \boldsymbol{y}^{1}\right)}{D_{o}^{0}\left(\overline{\boldsymbol{x}}, \boldsymbol{y}^{0}\right)} \frac{D_{o}^{0}\left(\boldsymbol{x}^{1}, \boldsymbol{y}^{0}\right)}{D_{o}^{0}\left(\boldsymbol{x}^{1}, \boldsymbol{y}^{1}\right)}\right)}_{\text {Fourth term Path A, OME }} \times \underbrace{\left(\frac{D_{o}^{1}\left(\overline{\boldsymbol{x}}, \boldsymbol{y}^{1}\right)}{D_{o}^{1}\left(\overline{\boldsymbol{x}}, \boldsymbol{y}^{0}\right)} \frac{D_{o}^{1}\left(\boldsymbol{x}^{1}, \boldsymbol{y}^{0}\right)}{D_{o}^{1}\left(\boldsymbol{x}^{1}, \boldsymbol{y}^{1}\right)}\right)}_{\text {Fourth term Path }, \text { OME }}]^{1 / 2}}_{\text {Fourth terms paths AC, OME }} \times \\
& \underbrace{[\underbrace{\left(\frac{D_{o}^{0}\left(\overline{\boldsymbol{x}}, \boldsymbol{y}^{1}\right)}{D_{o}^{0}\left(\overline{\boldsymbol{x}}, \boldsymbol{y}^{0}\right)} \frac{D_{o}^{0}\left(\boldsymbol{x}^{0}, \boldsymbol{y}^{0}\right)}{D_{o}^{0}\left(\boldsymbol{x}^{0}, \boldsymbol{y}^{1}\right)}\right)}_{\text {Fourth term Path B, OME }} \times \underbrace{\left(\frac{D_{o}^{1}\left(\overline{\boldsymbol{x}}, \boldsymbol{y}^{1}\right)}{D_{o}^{1}\left(\overline{\boldsymbol{x}}, \boldsymbol{y}^{0}\right)} \frac{D_{o}^{1}\left(\boldsymbol{x}^{0}, \boldsymbol{y}^{0}\right)}{D_{o}^{1}\left(\boldsymbol{x}^{0}, \boldsymbol{y}^{1}\right)}\right)}_{\text {Fourth term Path D, OME }}]^{1 / 2}}_{\text {Fourth terms paths BD, OME }},
\end{aligned}
$$

where the second and third rows correspond to the radial scale and input mix effect (gathering the third terms in the previous decompositions), and the fourth and fifth rows measure the output mix effect (gathering the fourth terms in the previous decompositions).

This decomposition can be obtained by setting in the deatfpmb (X, Y, ...) function the period parameter to (geomean). In the output of the function, the technical efficiency change is identified by EC, the geometric mean of the two technological change factors by TC, the geometric mean of the four scale and input mix effects by SEC, and the geometric mean of the four output mix effects by OME. Each specific component can be recovered from the previous decompositions under the (base) or (comparison) specification. The related decompositions can be obtained by changing the argument (complete) in the decomp parameter to (ccd) or (rts).

$>$ tfpmb_oo_geo_complete $=\operatorname{deatfpmb}(X, Y$, 'orient', 'oo', $\ldots$ 


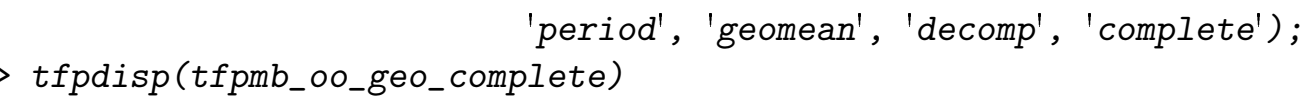

\begin{tabular}{|c|c|c|c|c|c|}
\hline DMU I & MB I & $\mathrm{ECl}$ & $\mathrm{TCl}$ & $\mathrm{SECl}$ & OME I \\
\hline 11 & $0.5185 \mid$ & $0.5833 \mid$ & $1.1055 \mid$ & $0.8097 \mid$ & 0.99301 \\
\hline 21 & $1.3181 \mid$ & 1.00001 & 1.3416 & $0.9868 \mid$ & 0.9956 \\
\hline 31 & $1.0000 \mid$ & 1.00001 & 1.00001 & 1.00001 & 1.00001 \\
\hline 41 & 2.50001 & $1.2500 \mid$ & 1.00001 & 2.00001 & 1.00001 \\
\hline 51 & $0.5765 \mid$ & 0.60001 & 1.00001 & 0.96081 & 1.00001 \\
\hline
\end{tabular}

MB $=$ Moorsteen-Bjurek. $\mathrm{EC}=$ Efficiency Change. $\mathrm{TC}=$ Technological Change. SEC = Scale Effect. OME = Output Mix Effect.

\subsection{The input orientated decomposition of MBPI}

The input orientated decomposition of the base period MBPI

The counterpart decomposition of the input orientated, base period MPI - see expression (17) - along 'Path E' is given by

$$
\begin{aligned}
M B^{0}\left(\boldsymbol{x}^{1}, \boldsymbol{y}^{1}, \boldsymbol{x}^{0}, \boldsymbol{y}^{0} ; \overline{\boldsymbol{x}}, \overline{\boldsymbol{y}}\right)= & E C_{i}\left(\boldsymbol{x}^{1}, \boldsymbol{y}^{1}, \boldsymbol{x}^{0}, \boldsymbol{y}^{0}\right) \times T C_{i}^{1,0}\left(\boldsymbol{x}^{1}, \boldsymbol{y}^{1}\right) \times \\
& \left(\frac{D_{i}^{0}\left(\boldsymbol{x}^{1}, \boldsymbol{y}^{0}\right)}{D_{i}^{0}\left(\boldsymbol{x}^{0}, \boldsymbol{y}^{0}\right)} \frac{D_{i}^{0}\left(\boldsymbol{x}^{0}, \overline{\boldsymbol{y}}\right)}{D_{i}^{0}\left(\boldsymbol{x}^{1}, \overline{\boldsymbol{y}}\right)}\right) \times\left(\frac{D_{o}^{0}\left(\overline{\boldsymbol{x}}, \boldsymbol{y}^{1}\right)}{D_{o}^{0}\left(\overline{\boldsymbol{x}}, \boldsymbol{y}^{0}\right)} \frac{D_{i}^{0}\left(\boldsymbol{x}^{1}, \boldsymbol{y}^{1}\right)}{D_{i}^{0}\left(\boldsymbol{x}^{1}, \boldsymbol{y}^{0}\right)}\right) .
\end{aligned}
$$

The factors on the right-hand side of the equality sign represent, respectively, technical efficiency change, technological change (conditional on $\left(\boldsymbol{x}^{1}, \boldsymbol{y}^{1}\right)$ ), the input mix effect (conditional on $\boldsymbol{y}^{0}$ ), and the radial scale and output mix effect (conditional on $\boldsymbol{x}^{1}$ ).

The counterpart to expression (18), along 'Path F', is

$$
\begin{aligned}
M B^{0}\left(\boldsymbol{x}^{1}, \boldsymbol{y}^{1}, \boldsymbol{x}^{0}, \boldsymbol{y}^{0} ; \overline{\boldsymbol{x}}, \overline{\boldsymbol{y}}\right)= & E C_{o}\left(\boldsymbol{x}^{1}, \boldsymbol{y}^{1}, \boldsymbol{x}^{0}, \boldsymbol{y}^{0}\right) \times T C_{o}^{1,0}\left(\boldsymbol{x}^{1}, \boldsymbol{y}^{1}\right) \times \\
& \left(\frac{D_{i}^{0}\left(\boldsymbol{x}^{1}, \boldsymbol{y}^{1}\right)}{D_{i}^{0}\left(\boldsymbol{x}^{0}, \boldsymbol{y}^{1}\right)} \frac{D_{i}^{0}\left(\boldsymbol{x}^{0}, \overline{\boldsymbol{y}}\right)}{D_{i}^{0}\left(\boldsymbol{x}^{1}, \overline{\boldsymbol{y}}\right)}\right) \times\left(\frac{D_{o}^{0}\left(\overline{\boldsymbol{x}}, \boldsymbol{y}^{1}\right)}{D_{o}^{0}\left(\overline{\boldsymbol{x}}, \boldsymbol{y}^{0}\right)} \frac{D_{i}^{0}\left(\boldsymbol{x}^{0}, \boldsymbol{y}^{1}\right)}{D_{i}^{0}\left(\boldsymbol{x}^{0}, \boldsymbol{y}^{0}\right)}\right) .
\end{aligned}
$$


Both decompositions can be calculated by setting the orient parameter to (io) in the $\operatorname{deatfpmb}(\mathrm{X}, \mathrm{Y}, \ldots)$ function.

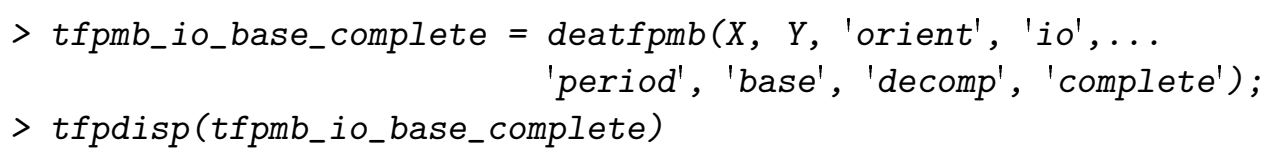

Total Factor Productivity (TFP)

DMUs : 5

Inputs: 2 Outputs: 2

Model: tfp-mb

Orientation: io (Input orientated)

Returns to scale: vrs (Variable)

Moorsteen-Bjurek index:

Reference period is base period

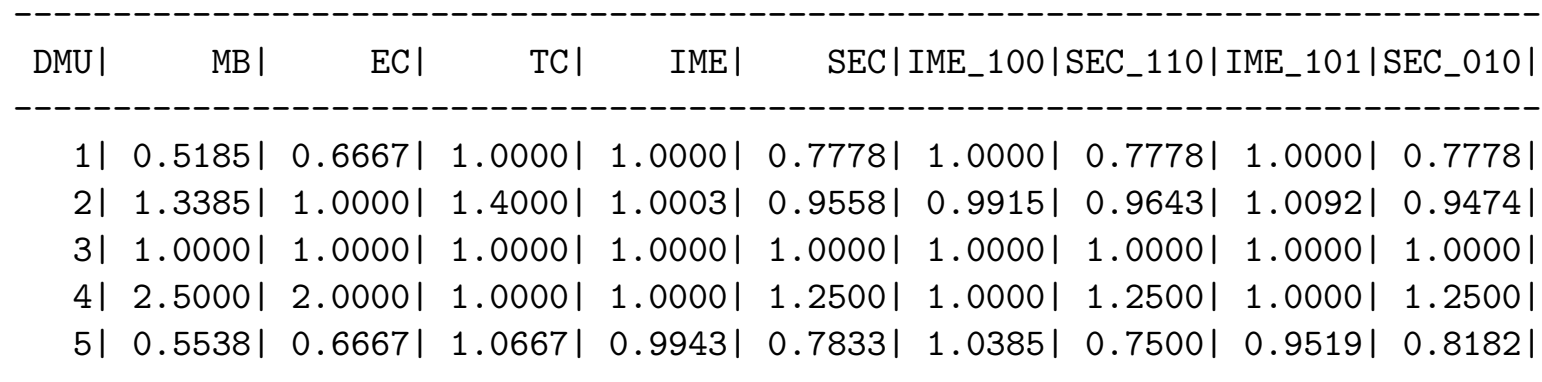

MB $=$ Moorsteen-Bjurek. $\mathrm{EC}=$ Efficiency Change. $\mathrm{TC}=$ Technological Change. IME $=$ Input Mix Effect. SEC = Scale Effect.

To obtain the related decompositions including the $C C D$ index or the constant-returns-toscale versions of expressions (38) and (39), the user changes the decomp parameter to (ccd) or (crs). In the last case, the fourth factor captures only the output mix effect as the radial scale component vanishes under the CRS specification.

The input orientated decomposition of the comparison period MBPI

The input orientated decomposition of the comparison period MBPI along 'Path G', comparable to expression (25), is

$$
\begin{aligned}
M B^{1}\left(\boldsymbol{x}^{1}, \boldsymbol{y}^{1}, \boldsymbol{x}^{0}, \boldsymbol{y}^{0} ; \overline{\boldsymbol{x}}, \overline{\boldsymbol{y}}\right)= & E C_{i}\left(\boldsymbol{x}^{1}, \boldsymbol{y}^{1}, \boldsymbol{x}^{0}, \boldsymbol{y}^{0}\right) \times T C_{i}^{1,0}\left(\boldsymbol{x}^{0}, \boldsymbol{y}^{0}\right) \times \\
& \left(\frac{D_{i}^{1}\left(\boldsymbol{x}^{1}, \boldsymbol{y}^{0}\right)}{D_{i}^{1}\left(\boldsymbol{x}^{0}, \boldsymbol{y}^{0}\right)} \frac{D_{i}^{1}\left(\boldsymbol{x}^{0}, \overline{\boldsymbol{y}}\right)}{D_{i}^{1}\left(\boldsymbol{x}^{1}, \overline{\boldsymbol{y}}\right)}\right) \times\left(\frac{D_{o}^{1}\left(\overline{\boldsymbol{x}}, \boldsymbol{y}^{1}\right)}{D_{o}^{1}\left(\overline{\boldsymbol{x}}, \boldsymbol{y}^{0}\right)} \frac{D_{i}^{1}\left(\boldsymbol{x}^{1}, \boldsymbol{y}^{1}\right)}{D_{i}^{1}\left(\boldsymbol{x}^{1}, \boldsymbol{y}^{0}\right)}\right),
\end{aligned}
$$


and along 'Path H', corresponding to expression (26),

$$
\begin{aligned}
M B^{1}\left(\boldsymbol{x}^{1}, \boldsymbol{y}^{1}, \boldsymbol{x}^{0}, \boldsymbol{y}^{0} ; \overline{\boldsymbol{x}}, \overline{\boldsymbol{y}}\right)= & E C_{o}\left(\boldsymbol{x}^{1}, \boldsymbol{y}^{1}, \boldsymbol{x}^{0}, \boldsymbol{y}^{0}\right) \times T C_{o}^{1,0}\left(\boldsymbol{x}^{0}, \boldsymbol{y}^{0}\right) \times \\
& \left(\frac{D_{i}^{1}\left(\boldsymbol{x}^{1}, \boldsymbol{y}^{1}\right)}{D_{i}^{1}\left(\boldsymbol{x}^{0}, \boldsymbol{y}^{1}\right)} \frac{D_{i}^{1}\left(\boldsymbol{x}^{0}, \overline{\boldsymbol{y}}\right)}{D_{i}^{1}\left(\boldsymbol{x}^{1}, \overline{\boldsymbol{y}}\right)}\right) \times\left(\frac{D_{o}^{1}\left(\overline{\boldsymbol{x}}, \boldsymbol{y}^{1}\right)}{D_{o}^{1}\left(\overline{\boldsymbol{x}}, \boldsymbol{y}^{0}\right)} \frac{D_{i}^{1}\left(\boldsymbol{x}^{0}, \boldsymbol{y}^{1}\right)}{D_{i}^{1}\left(\boldsymbol{x}^{0}, \boldsymbol{y}^{0}\right)}\right) .
\end{aligned}
$$

These decompositions are obtained by setting the period parameter to (comparison) in the deatfpmb (X, Y, ...) function. Changing the decomp parameter to (ccd) or (crs) yields the related decompositions.

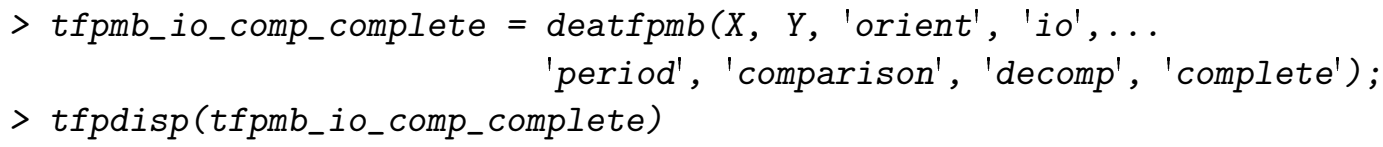

Total Factor Productivity (TFP)

DMUs : 5

Inputs: 2 Outputs: 2

Model: tfp-mb

Orientation: io (Input orientated)

Returns to scale: vrs (Variable)

Moorsteen-Bjurek index:

Reference period is comparison period

DMU I MBI ECI TC| IME I SEC|IME_100|SEC_110|IME_101|SEC_010 |

$1|0.5185| 0.6667|1.0000| 1.0000|0.7778| 1.0000|0.7778| 1.0000|0.7778|$

$2|1.2981| 1.0000|1.0000| 1.0102|1.2851| 0.9524|1.3630| 1.0714|1.2116|$

$3|1.0000| 1.0000|1.0000| 1.0000|1.0000| 1.0000|1.0000| 1.0000|1.0000|$

$4|2.5000| 2.0000|1.0000| 1.0000|1.2500| 1.0000|1.2500| 1.0000|1.2500|$

$5|0.6000| 0.6667|1.0000| 1.0607|0.8485| 1.1250|0.8000| 1.0000|0.9000|$

$M B=$ Moorsteen-Bjurek. $\mathrm{EC}=$ Efficiency Change. $\mathrm{TC}=$ Technological Change.

IME $=$ Input Mix Effect. SEC = Scale Effect.

The input orientated decomposition of the geometric mean MBPI

There are four decompositions of the productivity index $M B^{t}\left(\boldsymbol{x}^{1}, \boldsymbol{y}^{1}, \boldsymbol{x}^{0}, \boldsymbol{y}^{0} ; \overline{\boldsymbol{x}}, \overline{\boldsymbol{y}}\right)$ from the input orientation. For $t=0$, expressions (38) and (39) are decompositions of $M B^{0}\left(\boldsymbol{x}^{1}, \boldsymbol{y}^{1}, \boldsymbol{x}^{0}, \boldsymbol{y}^{0}\right.$; $\overline{\boldsymbol{x}}, \overline{\boldsymbol{y}})$. For $t=1$, expressions (40) and (41) are decompositions of $M B^{1}\left(\boldsymbol{x}^{1}, \boldsymbol{y}^{1}, \boldsymbol{x}^{0}, \boldsymbol{y}^{0} ; \overline{\boldsymbol{x}}, \overline{\boldsymbol{y}}\right)$. By taking the geometric mean of these four decompositions we obtain a decomposition of the geometric mean index $M B\left(\boldsymbol{x}^{1}, \boldsymbol{y}^{1}, \boldsymbol{x}^{0}, \boldsymbol{y}^{0} ; \overline{\boldsymbol{x}}, \overline{\boldsymbol{y}}\right)=\left[M B^{0}\left(\boldsymbol{x}^{1}, \boldsymbol{y}^{1}, \boldsymbol{x}^{0}, \boldsymbol{y}^{0} ; \overline{\boldsymbol{x}}, \overline{\boldsymbol{y}}\right) M B^{1}\left(\boldsymbol{x}^{1}, \boldsymbol{y}^{1}, \boldsymbol{x}^{0}, \boldsymbol{y}^{0} ;\right.\right.$ $\overline{\boldsymbol{x}}, \overline{\boldsymbol{y}})]^{1 / 2}$. This expression corresponds to expression (37), but based on input distance functions instead of output distance functions. 
This decomposition can be obtained by setting the period parameter to (geomean) in the $\operatorname{deatfpmb}(\mathrm{X}, \mathrm{Y}, \ldots)$ function, along with the (io) in orientation.

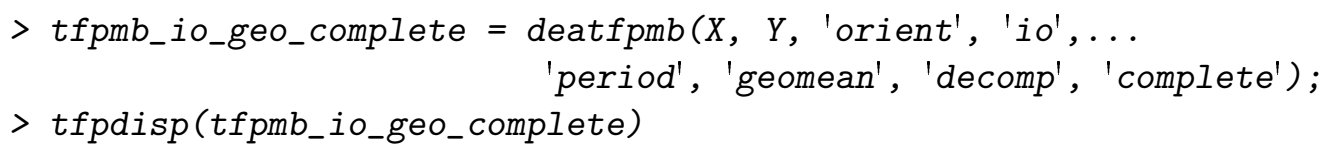

Total Factor Productivity (TFP)

DMUs : 5

Inputs: 2 Outputs: 2

Model: tfp-mb

Orientation: io (Input orientated)

Returns to scale: vrs (Variable)

Moorsteen-Bjurek index:

Reference period is Geometric mean

\begin{tabular}{rrrrrr} 
DMU I & MB | & EC | & TC I & IME | & SEC | \\
\hline $1 \mid$ & $0.5185 \mid$ & $0.6667 \mid$ & $1.0000 \mid$ & $1.0000 \mid$ & $0.7778 \mid$ \\
$2 \mid$ & $1.3181 \mid$ & $1.0000 \mid$ & $1.1832 \mid$ & $1.0052 \mid$ & $1.1083 \mid$ \\
$3 \mid$ & $1.0000 \mid$ & $1.0000 \mid$ & $1.0000 \mid$ & $1.0000 \mid$ & $1.0000 \mid$ \\
$4 \mid$ & $2.5000 \mid$ & $2.0000 \mid$ & $1.0000 \mid$ & $1.0000 \mid$ & $1.2500 \mid$ \\
$5 \mid$ & $0.5765 \mid$ & $0.6667 \mid$ & $1.0328 \mid$ & $1.0269 \mid$ & $0.8153 \mid$
\end{tabular}

$\mathrm{MB}=$ Moorsteen-Bjurek. $\mathrm{EC}=$ Efficiency Change. $\mathrm{TC}=$ Technological Change. IME = Input Mix Effect. SEC = Scale Effect.

\section{Price-Weighted Productivity Indices (Fisher)}

The generic definition of a price-weighted productivity index comparing $\left(\boldsymbol{x}^{1}, \boldsymbol{y}^{1}\right)$ to $\left(\boldsymbol{x}^{0}, \boldsymbol{y}^{0}\right)$, conditional on input prices $\boldsymbol{w}^{t}$ and output prices $\boldsymbol{p}^{t}$, is

$$
\operatorname{PROD}\left(\boldsymbol{x}^{1}, \boldsymbol{y}^{1}, \boldsymbol{x}^{0}, \boldsymbol{y}^{0} ; \boldsymbol{w}^{t}, \boldsymbol{p}^{t}\right) \equiv \frac{\boldsymbol{p}^{t} \cdot \boldsymbol{y}^{1} / \boldsymbol{w}^{t} \cdot \boldsymbol{x}^{1}}{\boldsymbol{p}^{t} \cdot \boldsymbol{y}^{0} / \boldsymbol{w}^{t} \cdot \boldsymbol{x}^{0}}=\frac{\boldsymbol{p}^{t} \cdot \boldsymbol{y}^{1} / \boldsymbol{p}^{t} \cdot \boldsymbol{y}^{0}}{\boldsymbol{w}^{t} \cdot \boldsymbol{x}^{1} / \boldsymbol{w}^{t} \cdot \boldsymbol{x}^{0}}
$$

This is the ratio of two productivity levels (of period 1 and period 0 , respectively), or the ratio of two quantity indices (for output and input, respectively). Period $t$ serves as benchmark period, determining the prices with which quantities are multiplied. For $t=0$ we obtain the Laspeyres productivity index, and for $t=1$ the Paasche productivity index. The geometric mean of the two is the Fisher productivity index.

To calculate these indices, users must provide not only input and output quantities ( $\mathrm{X}$ and $\mathrm{Y}$ ) but also corresponding prices ( $\mathrm{W}$ and $\mathrm{P}$ ), which are three-dimensional arrays, with the third 
dimension corresponding to the time periods $t=0,1, \ldots, T$. Note that in principle, the priceweighted indices could be calculated using any set of prices provided by the user. However, if prices are chosen as above, the toolbox calculates the Laspeyres, Paasche, and Fisher indices by default.

\subsection{The output orientated decomposition of a price-weighted productivity index}

The output orientated decomposition of the Laspeyres PI

Following 'Path A', the decomposition of expression (42) with $t=0$ reads

$$
\begin{aligned}
P R O D\left(\boldsymbol{x}^{1}, \boldsymbol{y}^{1}, \boldsymbol{x}^{0}, \boldsymbol{y}^{0} ; \boldsymbol{w}^{0}, \boldsymbol{p}^{0}\right)= & E C_{o}\left(\boldsymbol{x}^{1}, \boldsymbol{y}^{1}, \boldsymbol{x}^{0}, \boldsymbol{y}^{0}\right) \times T C_{o}^{1,0}\left(\boldsymbol{x}^{1}, \boldsymbol{y}^{1}\right) \times \\
& {\left[\frac{D_{o}^{0}\left(\boldsymbol{x}^{0}, \boldsymbol{y}^{0}\right)}{D_{o}^{0}\left(\boldsymbol{x}^{1}, \boldsymbol{y}^{0}\right)} \frac{\boldsymbol{w}^{0} \cdot \boldsymbol{x}^{0}}{\boldsymbol{w}^{0} \cdot \boldsymbol{x}^{1}}\right] \times\left[\frac{D_{o}^{0}\left(\boldsymbol{x}^{1}, \boldsymbol{y}^{0}\right)}{D_{o}^{0}\left(\boldsymbol{x}^{1}, \boldsymbol{y}^{1}\right)} \frac{\boldsymbol{p}^{0} \cdot \boldsymbol{y}^{1}}{\boldsymbol{p}^{0} \cdot \boldsymbol{y}^{0}}\right] . }
\end{aligned}
$$

As previously seen, the first factor after the equality measures technical efficiency change and the second factor measures technological change. Balk and Zofío (2018) show that the third factor corresponds to the scale (including input mix) effect, while the fourth factor corresponds to the output mix effect. Following the alternative 'Path B' delivers

$$
\begin{aligned}
P R O D\left(\boldsymbol{x}^{1}, \boldsymbol{y}^{1}, \boldsymbol{x}^{0}, \boldsymbol{y}^{0} ; \boldsymbol{w}^{0}, \boldsymbol{p}^{0}\right)= & E C_{o}\left(\boldsymbol{x}^{1}, \boldsymbol{y}^{1}, \boldsymbol{x}^{0}, \boldsymbol{y}^{0}\right) \times T C_{o}^{1,0}\left(\boldsymbol{x}^{1}, \boldsymbol{y}^{1}\right) \times \\
& {\left[\frac{D_{o}^{0}\left(\boldsymbol{x}^{0}, \boldsymbol{y}^{1}\right)}{D_{o}^{0}\left(\boldsymbol{x}^{1}, \boldsymbol{y}^{1}\right)} \frac{\boldsymbol{w}^{0} \cdot \boldsymbol{x}^{0}}{\boldsymbol{w}^{0} \cdot \boldsymbol{x}^{1}}\right] \times\left[\frac{D_{o}^{0}\left(\boldsymbol{x}^{0}, \boldsymbol{y}^{0}\right)}{D_{o}^{0}\left(\boldsymbol{x}^{0}, \boldsymbol{y}^{1}\right)} \frac{\boldsymbol{p}^{0} \cdot \boldsymbol{y}^{1}}{\boldsymbol{p}^{0} \cdot \boldsymbol{y}^{0}}\right] }
\end{aligned}
$$

which differs from expression (43) in scale effect and output mix effect. Taking the geometric mean of these two expressions yields

$$
\begin{gathered}
\operatorname{PROD}\left(\boldsymbol{x}^{1}, \boldsymbol{y}^{1}, \boldsymbol{x}^{0}, \boldsymbol{y}^{0} ; \boldsymbol{w}^{0}, \boldsymbol{p}^{0}\right)=E C_{o}\left(\boldsymbol{x}^{1}, \boldsymbol{y}^{1}, \boldsymbol{x}^{0}, \boldsymbol{y}^{0}\right) \times T C_{o}^{1,0}\left(\boldsymbol{x}^{1}, \boldsymbol{y}^{1}\right) \times \\
{\left[\left(\frac{D_{o}^{0}\left(\boldsymbol{x}^{0}, \boldsymbol{y}^{0}\right)}{D_{o}^{0}\left(\boldsymbol{x}^{1}, \boldsymbol{y}^{0}\right)} \frac{D_{o}^{0}\left(\boldsymbol{x}^{0}, \boldsymbol{y}^{1}\right)}{D_{o}^{0}\left(\boldsymbol{x}^{1}, \boldsymbol{y}^{1}\right)}\right)^{1 / 2} \frac{\boldsymbol{w}^{0} \cdot \boldsymbol{x}^{0}}{\boldsymbol{w}^{0} \cdot \boldsymbol{x}^{1}}\right] \times\left[\left(\frac{D_{o}^{0}\left(\boldsymbol{x}^{1}, \boldsymbol{y}^{0}\right)}{D_{o}^{0}\left(\boldsymbol{x}^{1}, \boldsymbol{y}^{1}\right)} \frac{D_{o}^{0}\left(\boldsymbol{x}^{0}, \boldsymbol{y}^{0}\right)}{D_{o}^{0}\left(\boldsymbol{x}^{0}, \boldsymbol{y}^{1}\right)}\right)^{1 / 2} \frac{\boldsymbol{p}^{0} \cdot \boldsymbol{y}^{1}}{\boldsymbol{p}^{0} \cdot \boldsymbol{y}^{0}}\right] .}
\end{gathered}
$$

This decomposition can be computed in MATLAB through the deatfprod (X, Y, ...) function. The orient parameter should be set to the output orientation (oo) and the period parameter to (base). The decomposition parameter decomp should be (complete).

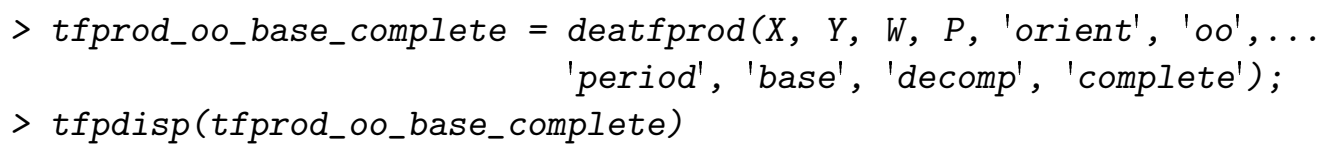

Total Factor Productivity (TFP)

DMUs : 5 


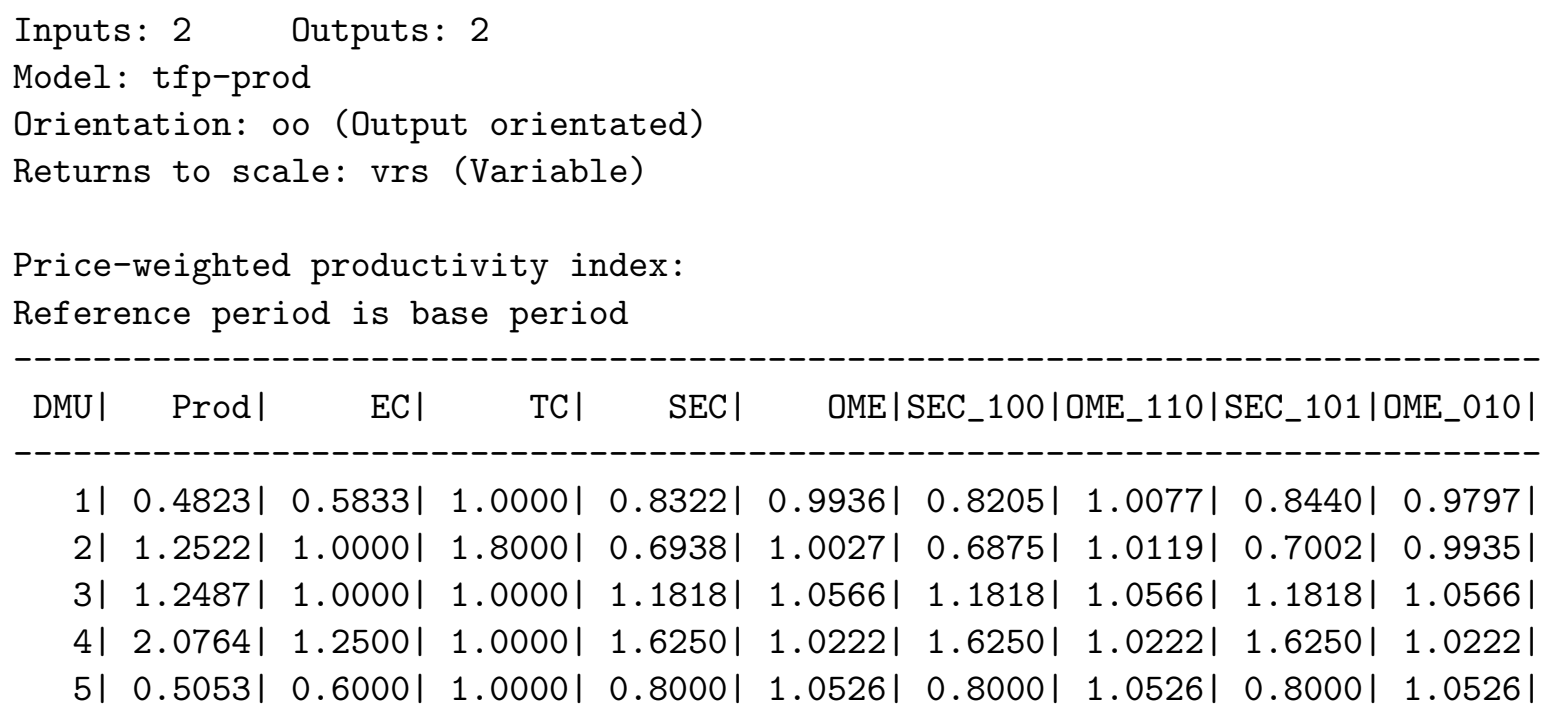

Prod $=$ Laspeyres. EC = Efficiency Change. $\mathrm{TC}=$ Technological Change. SEC = Scale Effect. OME = Output Mix Effect.

The output of the function successively shows the Laspeyres PI (PROD), the technical efficiency change factor (EC), the technological change factor (TC), the scale (including input mix) factor (SEC), and the output mix factor (OME). The last four columns show the separate values of the scale and output mix effects along 'Path A' and 'Path B'. They are identified by the time superscripts of the input and output arguments; e.g., SEC_100 identifies the third term in expression (43), which is the counterpart to $S E C_{o}^{0}\left(\boldsymbol{x}^{1}, \boldsymbol{x}^{0}, \boldsymbol{y}^{0}\right)$ in expression (3).

The distance functions may be infeasible, and therefore some of the factors in the above decompositions cannot be calculated. This is why the toolbox directly calculates the Laspeyres PI.

\section{Related decompositions of the Laspeyres PI}

Using expression (6), defining the output orientated, base period $C C D$ index, expression (43) can be simplified to

$$
\begin{aligned}
P R O D\left(\boldsymbol{x}^{1}, \boldsymbol{y}^{1}, \boldsymbol{x}^{0}, \boldsymbol{y}^{0} ; \boldsymbol{w}^{0}, \boldsymbol{p}^{0}\right)= & M_{o}^{0}\left(x^{1}, y^{1}, x^{0}, y^{0}\right) \times \\
& {\left[\frac{D_{o}^{0}\left(\boldsymbol{x}^{0}, \boldsymbol{y}^{0}\right)}{D_{o}^{0}\left(\boldsymbol{x}^{1}, \boldsymbol{y}^{0}\right)} \frac{\boldsymbol{w}^{0} \cdot \boldsymbol{x}^{0}}{\boldsymbol{w}^{0} \cdot \boldsymbol{x}^{1}}\right] \times\left[\frac{D_{o}^{0}\left(\boldsymbol{x}^{1}, \boldsymbol{y}^{0}\right)}{D_{o}^{0}\left(\boldsymbol{x}^{1}, \boldsymbol{y}^{1}\right)} \frac{\boldsymbol{p}^{0} \cdot \boldsymbol{y}^{1}}{\boldsymbol{p}^{0} \cdot \boldsymbol{y}^{0}}\right] . }
\end{aligned}
$$

The same holds for expressions (44) and (45). To obtain such a decomposition, the user should set the decomp parameter to (ccd).

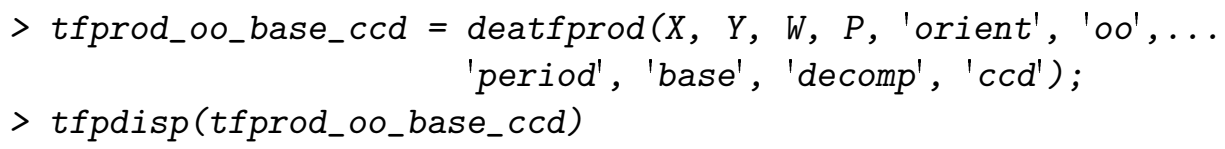

Total Factor Productivity (TFP) 
DMUs : 5

Inputs: 2 Outputs: 2

Model: tfp-prod

Orientation: oo (Output orientated)

Returns to scale: vrs (Variable)

Price-weighted productivity index:

Reference period is base period

\begin{tabular}{|c|c|c|c|c|c|c|c|c|}
\hline DMU I & Prod I & Mvrs I & $\mathrm{SECl}$ & OME I & SEC_100I & OME_110I & SEC_101I & OME_010I \\
\hline 11 & $0.4 \varepsilon$ & 0.006 & 0.83221 & $0.9936 \mid$ & $0.8205 \mid$ & $1.0077 \mid$ & 0.84401 & $0.9797 \mid$ \\
\hline 21 & 1.2522 & $1.8000 \mid$ & $0.6938 \mid$ & $1.0027 \mid$ & $0.6875 \mid$ & $1.0119 \mid$ & 0.70021 & $0.9935 \mid$ \\
\hline 31 & $1.2487 \mid$ & 1.00001 & $1.1818 \mid$ & $1.0566 \mid$ & $1.1818 \mid$ & $1.0566 \mid$ & $1.1818 \mid$ & $1.0566 \mid$ \\
\hline 41 & $2.0764 \mid$ & $1.2500 \mid$ & 1.62501 & $1.0222 \mid$ & $1.6250 \mid$ & $1.0222 \mid$ & 1.62501 & $1.0222 \mid$ \\
\hline 51 & 0.50531 & 0.60001 & 0.80001 & $1.0526 \mid$ & 0.80001 & $1.0526 \mid$ & 0.80001 & $1.0526 \mid$ \\
\hline
\end{tabular}

Prod = Laspeyres. Mvrs = Malmquist VRS. SEC = Scale Effect.

OME = Output Mix Effect.

If the base period technology is characterized by constant returns to scale, which can be tested by using the function deatestrtsm(X, Y, ...) with the orient parameter set to (oo), the first decomposition of the Laspeyres PI becomes

$$
\begin{aligned}
P R O D\left(\boldsymbol{x}^{1}, \boldsymbol{y}^{1}, \boldsymbol{x}^{0}, \boldsymbol{y}^{0} ; \boldsymbol{w}^{0}, \boldsymbol{p}^{0}\right)= & \check{M}_{o}^{0}\left(\boldsymbol{x}^{1}, \boldsymbol{y}^{1}, \boldsymbol{x}^{0}, \boldsymbol{y}^{0}\right) \times \\
& {\left[\frac{\check{D}_{o}^{0}\left(\boldsymbol{x}^{0}, \boldsymbol{y}^{0}\right)}{\check{D}_{o}^{0}\left(\boldsymbol{x}^{1}, \boldsymbol{y}^{0}\right)} \frac{\boldsymbol{w}^{0}}{\boldsymbol{w}^{0} \cdot \boldsymbol{x}^{1}}\right] \times\left[\frac{\check{D}_{o}^{0}\left(\boldsymbol{x}^{1}, \boldsymbol{y}^{0}\right)}{\check{D}_{o}^{0}\left(\boldsymbol{x}^{1}, \boldsymbol{y}^{1}\right)} \frac{\boldsymbol{p}^{0} \cdot \boldsymbol{y}^{1}}{\boldsymbol{p}^{0}}\right] . }
\end{aligned}
$$

For this version, the parameter decomp must be set to (crs). The third factor at the righthand side now measures only the input mix effect because the CRS specification eliminates the scale effect.

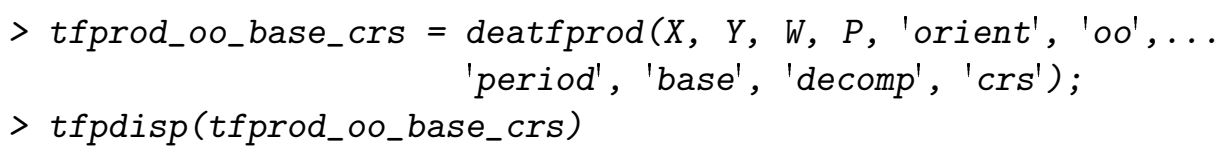

Total Factor Productivity (TFP)

DMUs : 5

Inputs: 2 Outputs: 2

Model: tfp-prod

Orientation: oo (Output orientated)

Returns to scale: crs (Constant)

Price-weighted productivity index: 
Reference period is base period

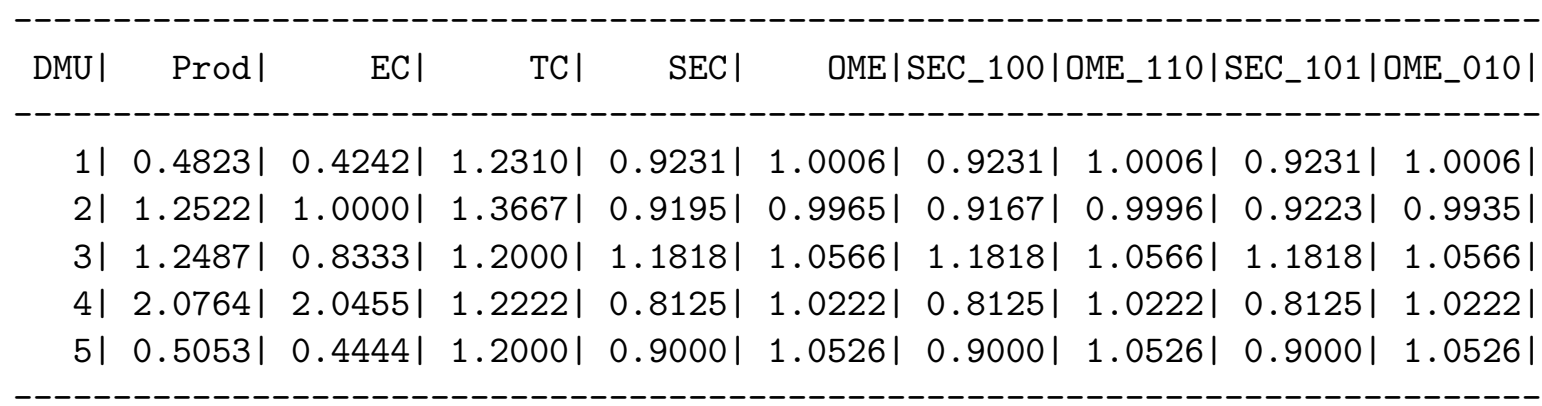

Prod $=$ Laspeyres. EC = Efficiency Change. $\mathrm{TC}=$ Technological Change.

SEC = Scale Effect. OME = Output Mix Effect.

Under CRS the SEC factor measures only the input mix effect.

The output orientated decomposition of the Paasche PI

Following 'Path C' and 'Path D' and combining the resulting decompositions, the decomposition of expression (42) with $t=1$ reads

$$
\begin{gathered}
\operatorname{PROD}\left(\boldsymbol{x}^{1}, \boldsymbol{y}^{1}, \boldsymbol{x}^{0}, \boldsymbol{y}^{0} ; \boldsymbol{w}^{1}, \boldsymbol{p}^{1}\right)=E C_{o}\left(\boldsymbol{x}^{1}, \boldsymbol{y}^{1}, \boldsymbol{x}^{0}, \boldsymbol{y}^{0}\right) \times T C_{o}^{1,0}\left(\boldsymbol{x}^{0}, \boldsymbol{y}^{0}\right) \times \\
{\left[\left(\frac{D_{o}^{1}\left(\boldsymbol{x}^{0}, \boldsymbol{y}^{0}\right)}{D_{o}^{1}\left(\boldsymbol{x}^{1}, \boldsymbol{y}^{0}\right)} \frac{D_{o}^{1}\left(\boldsymbol{x}^{0}, \boldsymbol{y}^{1}\right)}{D_{o}^{1}\left(\boldsymbol{x}^{1}, \boldsymbol{y}^{1}\right)}\right)^{1 / 2} \frac{\boldsymbol{w}^{1} \cdot \boldsymbol{x}^{0}}{\boldsymbol{w}^{1} \cdot \boldsymbol{x}^{1}}\right] \times\left[\left(\frac{D_{o}^{1}\left(\boldsymbol{x}^{1}, \boldsymbol{y}^{0}\right)}{D_{o}^{1}\left(\boldsymbol{x}^{1}, \boldsymbol{y}^{1}\right)} \frac{D_{o}^{1}\left(\boldsymbol{x}^{0}, \boldsymbol{y}^{0}\right)}{D_{o}^{1}\left(\boldsymbol{x}^{0}, \boldsymbol{y}^{1}\right)}\right)^{1 / 2} \frac{\boldsymbol{p}^{1} \cdot \boldsymbol{y}^{1}}{\boldsymbol{p}^{1} \cdot \boldsymbol{y}^{0}}\right],}
\end{gathered}
$$

This is the counterpart to expression (45). To obtain this decomposition the period parameter must be set to (comparison) in the deatfprod (X, Y, ...) function. Some factors may not be able to be calculated because of infeasibilities. The toolbox calculates the Paasche PI directly rather than as a product of the components.

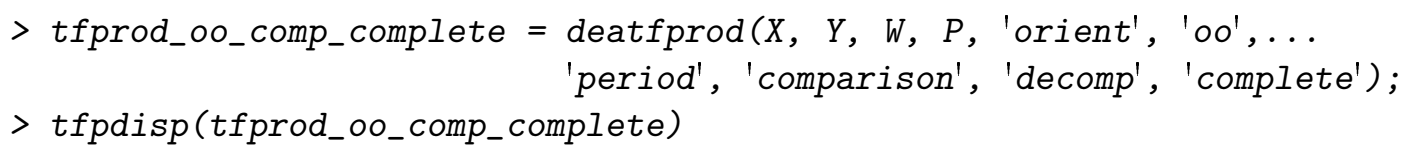

Total Factor Productivity (TFP)

DMUs: 5

Inputs: 2 Outputs: 2

Model: tfp-prod

Orientation: oo (Output orientated)

Returns to scale: vrs (Variable)

Price-weighted productivity index:

Reference period is comparison period 


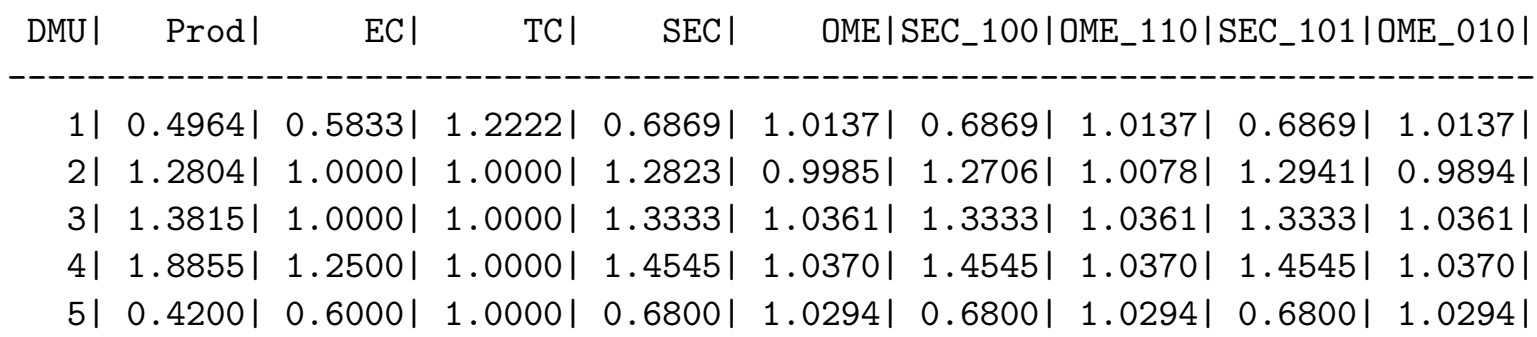

Prod $=$ Paasche. EC = Efficiency Change. $\mathrm{TC}=$ Technological Change. $\mathrm{SEC}=$ Scale Effect. OME = Output Mix Effect.

The first two factors together correspond to the output orientated, comparison period $C C D$ index $M_{o}^{1}\left(x^{1}, y^{1}, x^{0}, y^{0}\right)$, defined by expression (13). The related decomposition can be obtained by changing the decomp parameter from (complete) to (ccd). Also, if the existence of constant returns to scale for the compariaon period technology cannot be rejected, then the decomposition with $\check{M}_{o}^{1}\left(x^{1}, y^{1}, x^{0}, y^{0}\right)$ can be obtained by setting the decomp parameter to (crs).

The output orientated decomposition of the Fisher PI

The Fisher PI is the geometric mean of the Laspeyres and Paasche PIs. By merging expressions (45) and (48) its decomposition is

$$
\begin{gathered}
\operatorname{PROD}\left(\boldsymbol{x}^{1}, \boldsymbol{y}^{1}, \boldsymbol{x}^{0}, \boldsymbol{y}^{0} ; \boldsymbol{w}^{1}, \boldsymbol{p}^{1}, \boldsymbol{w}^{0}, \boldsymbol{p}^{0}\right) \equiv \frac{Q^{F}\left(\boldsymbol{p}^{1}, \boldsymbol{y}^{1}, \boldsymbol{p}^{0}, \boldsymbol{y}^{0}\right)}{Q^{F}\left(\boldsymbol{w}^{1}, \boldsymbol{x}^{1}, \boldsymbol{w}^{0}, \boldsymbol{x}^{0}\right)}= \\
{\left[M_{o}^{0}\left(\boldsymbol{x}^{1}, \boldsymbol{y}^{1}, \boldsymbol{x}^{0}, \boldsymbol{y}^{0}\right) M_{o}^{1}\left(\boldsymbol{x}^{1}, y^{1}, \boldsymbol{x}^{0}, \boldsymbol{y}^{0}\right)\right]^{1 / 2} \times} \\
{\left[\left(\frac{D_{o}^{0}\left(\boldsymbol{x}^{0}, \boldsymbol{y}^{0}\right)}{D_{o}^{0}\left(\boldsymbol{x}^{1}, \boldsymbol{y}^{0}\right)} \frac{D_{o}^{0}\left(\boldsymbol{x}^{0}, \boldsymbol{y}^{1}\right)}{D_{o}^{0}\left(\boldsymbol{x}^{1}, \boldsymbol{y}^{1}\right)} \frac{D_{o}^{1}\left(\boldsymbol{x}^{0}, \boldsymbol{y}^{0}\right)}{D_{o}^{1}\left(\boldsymbol{x}^{1}, \boldsymbol{y}^{0}\right)} \frac{D_{o}^{1}\left(\boldsymbol{x}^{0}, \boldsymbol{y}^{1}\right)}{D_{o}^{1}\left(\boldsymbol{x}^{1}, \boldsymbol{y}^{1}\right)}\right)^{1 / 4} \frac{1}{Q^{F}\left(\boldsymbol{w}^{1}, \boldsymbol{x}^{1}, \boldsymbol{w}^{0}, \boldsymbol{x}^{0}\right)}\right] \times} \\
{\left[\left(\frac{D_{o}^{0}\left(\boldsymbol{x}^{1}, \boldsymbol{y}^{0}\right)}{D_{o}^{0}\left(\boldsymbol{x}^{1}, \boldsymbol{y}^{1}\right)} \frac{D_{o}^{0}\left(\boldsymbol{x}^{0}, \boldsymbol{y}^{0}\right)}{D_{o}^{0}\left(\boldsymbol{x}^{0}, \boldsymbol{y}^{1}\right)} \frac{D_{o}^{1}\left(\boldsymbol{x}^{1}, \boldsymbol{y}^{0}\right)}{D_{o}^{1}\left(\boldsymbol{x}^{1}, \boldsymbol{y}^{1}\right)} \frac{D_{o}^{1}\left(\boldsymbol{x}^{0}, \boldsymbol{y}^{0}\right)}{D_{o}^{1}\left(\boldsymbol{x}^{0}, \boldsymbol{y}^{1}\right)}\right)^{1 / 4} Q^{F}\left(\boldsymbol{p}^{1}, \boldsymbol{y}^{1}, \boldsymbol{p}^{0}, \boldsymbol{y}^{0}\right)\right]}
\end{gathered}
$$

where $Q^{F}\left(\boldsymbol{p}^{1}, \boldsymbol{y}^{1}, \boldsymbol{p}^{0}, \boldsymbol{y}^{0}\right) \equiv\left[\left(\boldsymbol{p}^{0} \cdot \boldsymbol{y}^{1} / \boldsymbol{p}^{0} \cdot \boldsymbol{y}^{0}\right)\left(\boldsymbol{p}^{1} \cdot \boldsymbol{y}^{1} / \boldsymbol{p}^{1} \cdot \boldsymbol{y}^{0}\right)\right]^{1 / 2}$ is the Fisher output quantity index, and $Q^{F}\left(\boldsymbol{w}^{1}, \boldsymbol{x}^{1}, \boldsymbol{w}^{0}, \boldsymbol{x}^{0}\right) \equiv\left[\left(\boldsymbol{w}^{0} \cdot \boldsymbol{x}^{1} / \boldsymbol{w}^{0} \cdot \boldsymbol{x}^{0}\right)\left(\boldsymbol{w}^{1} \cdot \boldsymbol{x}^{1} / \boldsymbol{w}^{1} \cdot \boldsymbol{x}^{0}\right)\right]^{1 / 2}$ the Fisher input quantity index. In the above expression the geometric mean of the base and comparison period $C C D$ indices combines the technical efficiency change factor $E C_{o}\left(\boldsymbol{x}^{1}, \boldsymbol{y}^{1}, \boldsymbol{x}^{0}, \boldsymbol{y}^{0}\right)$ with the geometric mean of two technological change factors, $\left[T C_{o}^{1,0}\left(\boldsymbol{x}^{1}, \boldsymbol{y}^{1}\right) \times T C_{o}^{1,0}\left(\boldsymbol{x}^{0}, \boldsymbol{y}^{0}\right)\right]^{1 / 2}$.

The detailed decomposition is returned by the toolbox by calling the deatfprod $(\mathrm{X}, \mathrm{Y}, \ldots)$ function with the period parameter set to (geomean), the orientation parameter set to (oo), and the decomp parameter to (complete).

The output of the function successively shows the Fisher PI (PROD), the technical efficiency change factor, EC, the geometric mean of the technological change factors (TC), the geometric mean of the four separate scale effects as presented in the third row of expression (49) (SEC), and the geometric mean of the four separate output mix effects as presented in the fourth row of the same expression (OME). Each of these four separate terms in SEC or OME corresponds to 
a specific Path A, B, C or D, which can be recovered by running the base and comparison period decompositions as explained above. To ensure that the Fisher index is reported, regardless of the fact that some distance function values may be infeasible to compute, the toolbox calculates the geometric mean of the Laspeyres and Paasche PIs.

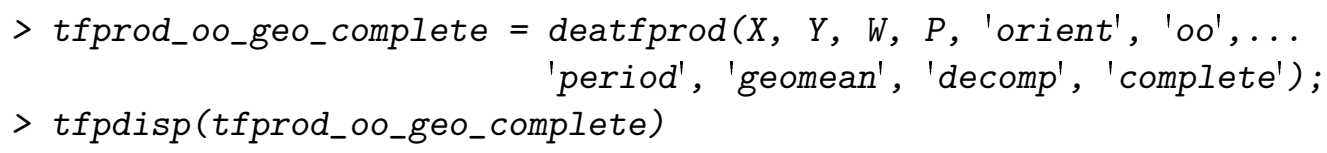

\begin{tabular}{|c|c|c|c|c|c|}
\hline DMUI & Prod I & $\mathrm{ECl}$ & $\mathrm{TCl}$ & $\mathrm{SECl}$ & OME I \\
\hline 11 & $0.4893 \mid$ & $0.5833 \mid$ & 1.1055 & 0.75601 & 1.0036 \\
\hline 21 & $1.2663 \mid$ & 1.00001 & 1.3416 & 0.94321 & 1.00061 \\
\hline 31 & $1.3134 \mid$ & 1.00001 & 1.00001 & $1.2553 \mid$ & $1.0463 \mid$ \\
\hline 41 & $1.9787 \mid$ & 1.2500 & 1.00001 & $1.5374 \mid$ & 1.0296 \\
\hline 51 & $0.4607 \mid$ & 0.60001 & 1.00001 & $0.7376 \mid$ & 1.0410 \\
\hline
\end{tabular}

Prod $=$ Fisher. EC = Efficiency Change. TC $=$ Technological Change .

$\mathrm{SEC}=$ Scale Effect. OME = Output Mix Effect.

Decomposition (49) is obtained by changing the decomp parameter to (ccd). Its constant returns to scale counterpart, once the deatestrtsm (X, Y, ...) function supports this for both technologies, is obtained by setting the parameter to (crs).

\subsection{The input orientated decomposition of a price-weighted productivity index}

The Laspeyres, Paasche, and Fisher productivity indices can also be decomposed from the input orientated perspective. This results in decompositions which are equivalent to those presented above.

The input orientated decomposition of the Laspeyres PI

Along 'Path E' and 'Path F' one obtains decompositions similar to expressions (43) and (44), except that input distance functions are used instead of output distance functions. The geometric mean, corresponding to expression (45), is 


$$
\begin{gathered}
\operatorname{PROD}\left(\boldsymbol{x}^{1}, \boldsymbol{y}^{1}, \boldsymbol{x}^{0}, \boldsymbol{y}^{0} ; \boldsymbol{w}^{0}, \boldsymbol{p}^{0}\right)=E C_{i}\left(\boldsymbol{x}^{1}, \boldsymbol{y}^{1}, \boldsymbol{x}^{0}, \boldsymbol{y}^{0}\right) \times T C_{i}^{1,0}\left(\boldsymbol{x}^{1}, \boldsymbol{y}^{1}\right) \times \\
{\left[\left(\frac{D_{i}^{0}\left(\boldsymbol{x}^{1}, \boldsymbol{y}^{0}\right)}{D_{i}^{0}\left(\boldsymbol{x}^{0}, \boldsymbol{y}^{0}\right)} \frac{D_{i}^{0}\left(\boldsymbol{x}^{1}, \boldsymbol{y}^{1}\right)}{D_{i}^{0}\left(\boldsymbol{x}^{0}, \boldsymbol{y}^{1}\right)}\right)^{1 / 2} \frac{\boldsymbol{w}^{0} \cdot \boldsymbol{x}^{0}}{\boldsymbol{w}^{0} \cdot \boldsymbol{x}^{1}}\right] \times\left[\left(\frac{D_{i}^{0}\left(\boldsymbol{x}^{1}, \boldsymbol{y}^{1}\right)}{D_{i}^{0}\left(\boldsymbol{x}^{1}, \boldsymbol{y}^{0}\right)} \frac{D_{i}^{0}\left(\boldsymbol{x}^{0}, \boldsymbol{y}^{1}\right)}{D_{i}^{0}\left(\boldsymbol{x}^{0}, \boldsymbol{y}^{0}\right)}\right)^{1 / 2} \frac{\boldsymbol{p}^{0} \cdot \boldsymbol{y}^{1}}{\boldsymbol{p}^{0} \cdot \boldsymbol{y}^{0}}\right] .}
\end{gathered}
$$

The first two factors capture technical efficiency change and technological change, respectively. The third and fourth factors measure the input mix effect and the scale (including output mix) effect, respectively.

This decomposition can be computed in MATLAB through the deatfprod $(\mathrm{X}, \mathrm{Y}, \ldots)$ function by setting the orient parameter to the input orientation (io), setting the period parameter to (base), and setting the decomposition parameter decomp to (complete). The output of the function yields the Laspeyres PI (PROD), the technical efficiency change factor (EC), the technological change factor (TC), and the geometric means and separate values of the input mix effects and scale effects corresponding to 'Path E' and 'Path F' above. The geometric means are identified by IME and SEC, respectively. Separate values are identified by the time superscripts of the input and output arguments of each factor. For instance, IME_100 identifies the first part of the third factor in (50),

$$
\frac{D_{i}^{0}\left(\boldsymbol{x}^{1}, \boldsymbol{y}^{0}\right)}{D_{i}^{0}\left(\boldsymbol{x}^{0}, \boldsymbol{y}^{0}\right)} \frac{\boldsymbol{w}^{0} \cdot \boldsymbol{x}^{0}}{\boldsymbol{w}^{0} \cdot \boldsymbol{x}^{1}}
$$

This is the counterpart to $I M E^{0}\left(\boldsymbol{x}^{1}, \boldsymbol{x}^{0}, \boldsymbol{y}^{0}\right)$ in expression (17).

Some distance function values may be impossible to compute, and therefore for certain observations some of the factors in the decompositions cannot be presented. In this case, the toolbox calculates the Laspeyres PI directly.

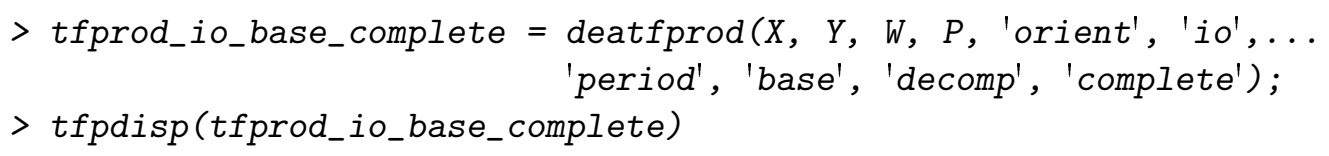

Total Factor Productivity (TFP)

DMUs : 5

Inputs: 2 Outputs: 2

Model: tfp-prod

Orientation: io (Input orientated)

Returns to scale: vrs (Variable)

Price-weighted productivity index:

Reference period is base period

DMU I Prodl EC| TC| IME I SEC|IME_100|SEC_110|IME_101|SEC_010|


$1|0.4823| 0.6667|1.0000| 0.9231|0.7838| 0.9231|0.7838| 0.9231|0.7838|$

$2|1.2522| 1.0000|1.4000| 0.9248|0.9672| 0.9167|0.9758| 0.9330|0.9586|$

$3|1.2487| 1.0000|1.0000| 1.1818|1.0566| 1.1818|1.0566| 1.1818|1.0566|$

$4|2.0764| 2.0000|1.0000| 0.8125|1.2778| 0.8125|1.2778| 0.8125|1.2778|$

$5|0.5053| 0.6667|1.0667| 0.8617|0.8246| 0.9000|0.7895| 0.8250|0.8612|$

Prod $=$ Laspeyres. EC = Efficiency Change. $\mathrm{TC}=$ Technological Change.

IME $=$ Input Mix Effect. SEC = Scale Effect.

To have the input orientated base period $C C D$ index $M_{i}^{0}\left(\boldsymbol{x}^{1}, \boldsymbol{y}^{1}, \boldsymbol{x}^{0}, \boldsymbol{y}^{0}\right)$ as part of the decomposition, the decomp parameter must be set to (ccd). If constant returns to scale holds for the base period technology (determined after running the function deatestrtsm(X, Y, ...) with the orient parameter set to (io)), then setting the decomp parameter to (crs) provides the correct decomposition. In this case, the third factor measures the input mix effect, but the fourth factor measures only the output mix effect since under CRS the scale effect vanishes.

The input orientated decomposition of the Paasche PI

By combining the results of following 'Path G' and 'Path H' we obtain the comparison period equivalent of expression (50), which is a decomposition of the Paasche PI,

$$
\begin{gathered}
P R O D\left(\boldsymbol{x}^{1}, \boldsymbol{y}^{1}, \boldsymbol{x}^{0}, \boldsymbol{y}^{0} ; \boldsymbol{w}^{1}, \boldsymbol{p}^{1}\right)=E C_{i}\left(\boldsymbol{x}^{1}, \boldsymbol{y}^{1}, \boldsymbol{x}^{0}, \boldsymbol{y}^{0}\right) \times T C_{i}^{1,0}\left(\boldsymbol{x}^{0}, \boldsymbol{y}^{0}\right) \times \\
{\left[\left(\frac{D_{i}^{1}\left(\boldsymbol{x}^{1}, \boldsymbol{y}^{0}\right)}{D_{i}^{1}\left(\boldsymbol{x}^{0}, \boldsymbol{y}^{0}\right)} \frac{D_{i}^{1}\left(\boldsymbol{x}^{1}, \boldsymbol{y}^{1}\right)}{D_{i}^{1}\left(\boldsymbol{x}^{0}, \boldsymbol{y}^{1}\right)}\right)^{1 / 2} \frac{\boldsymbol{w}^{1} \cdot \boldsymbol{x}^{0}}{\boldsymbol{w}^{1} \cdot \boldsymbol{x}^{1}}\right] \times\left[\left(\frac{D_{i}^{1}\left(\boldsymbol{x}^{1}, \boldsymbol{y}^{1}\right)}{D_{i}^{1}\left(\boldsymbol{x}^{1}, \boldsymbol{y}^{0}\right)} \frac{D_{i}^{1}\left(\boldsymbol{x}^{0}, \boldsymbol{y}^{1}\right)}{D_{i}^{1}\left(\boldsymbol{x}^{0}, \boldsymbol{y}^{0}\right)}\right)^{1 / 2} \frac{\boldsymbol{p}^{1} \cdot \boldsymbol{y}^{1}}{\boldsymbol{p}^{1} \cdot \boldsymbol{y}^{0}}\right],}
\end{gathered}
$$

whose terms are interpreted in the same way as before. This decomposition can be computed by setting the period parameter to (comparison) in the deatfprod(X, Y, ...) function. The related decompositions require setting the decomp parameter to (ccd) or (crs).

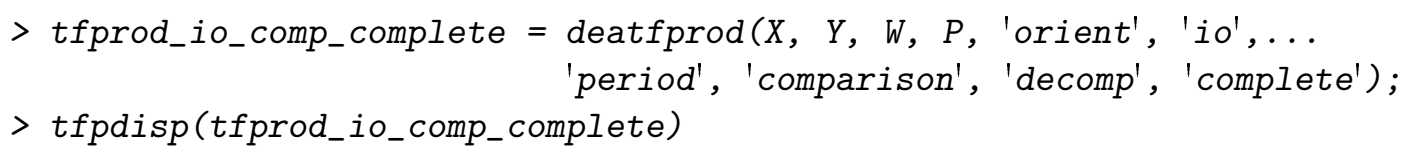

Total Factor Productivity (TFP)

DMUs : 5

Inputs: 2 Outputs: 2

Model: tfp-prod

Orientation: io (Input orientated)

Returns to scale: vrs (Variable)

Price-weighted productivity index: 
Reference period is comparison period

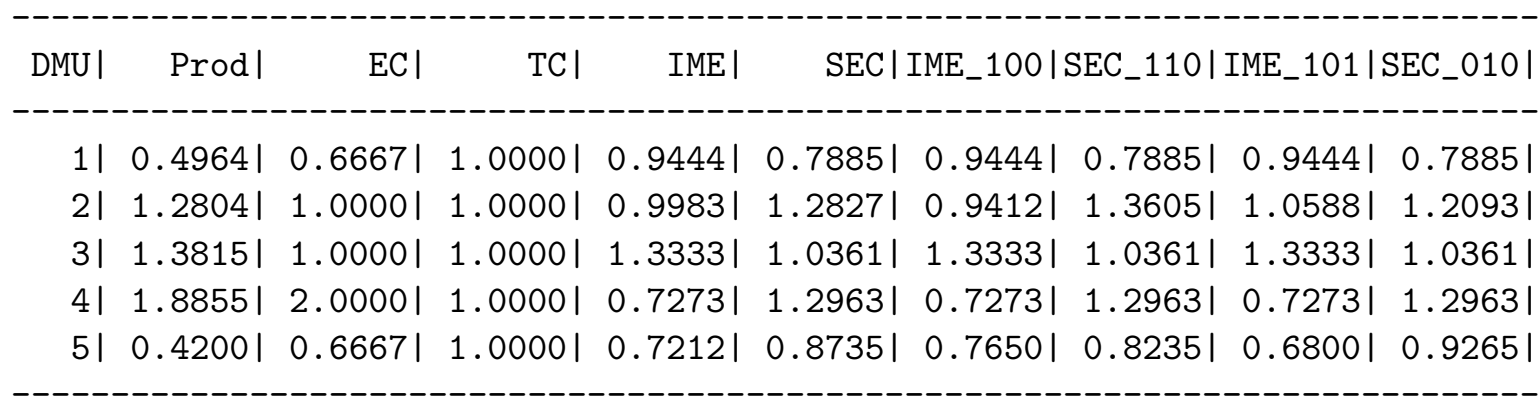

Prod $=$ Paasche. EC = Efficiency Change. $\mathrm{TC}=$ Technological Change .

IME $=$ Input Mix Effect. SEC = Scale Effect.

The input orientated decomposition of the Fisher PI

By taking the geometric mean of the decompositions (50) and (51) we obtain a decomposition of the Fisher PI,

$$
\begin{gathered}
P R O D\left(\boldsymbol{x}^{1}, \boldsymbol{y}^{1}, \boldsymbol{x}^{0}, \boldsymbol{y}^{0} ; \boldsymbol{w}^{1}, \boldsymbol{p}^{1}, \boldsymbol{w}^{0}, \boldsymbol{p}^{0}\right) \equiv \frac{Q^{F}\left(\boldsymbol{p}^{1}, \boldsymbol{y}^{1}, \boldsymbol{p}^{0}, \boldsymbol{y}^{0}\right)}{Q^{F}\left(\boldsymbol{w}^{1}, \boldsymbol{x}^{1}, \boldsymbol{w}^{0}, \boldsymbol{x}^{0}\right)}= \\
{\left[M_{i}^{0}\left(\boldsymbol{x}^{1}, \boldsymbol{y}^{1}, \boldsymbol{x}^{0}, \boldsymbol{y}^{0}\right) M_{i}^{1}\left(\boldsymbol{x}^{1}, y^{1}, \boldsymbol{x}^{0}, \boldsymbol{y}^{0}\right)\right]^{1 / 2} \times} \\
{\left[\left(\frac{D_{i}^{0}\left(\boldsymbol{x}^{1}, \boldsymbol{y}^{0}\right)}{D_{i}^{0}\left(\boldsymbol{x}^{0}, \boldsymbol{y}^{0}\right)} \frac{D_{i}^{0}\left(\boldsymbol{x}^{1}, \boldsymbol{y}^{1}\right)}{D_{i}^{0}\left(\boldsymbol{x}^{0}, \boldsymbol{y}^{1}\right)} \frac{D_{i}^{1}\left(\boldsymbol{x}^{1}, \boldsymbol{y}^{0}\right)}{D_{i}^{1}\left(\boldsymbol{x}^{0}, \boldsymbol{y}^{0}\right)} \frac{D_{i}^{1}\left(\boldsymbol{x}^{1}, \boldsymbol{y}^{1}\right)}{D_{i}^{1}\left(\boldsymbol{x}^{0}, \boldsymbol{y}^{1}\right)}\right)^{1 / 4} \frac{1}{Q^{F}\left(\boldsymbol{w}^{1}, \boldsymbol{x}^{1}, \boldsymbol{w}^{0}, \boldsymbol{x}^{0}\right)}\right] \times} \\
{\left[\left(\frac{D_{i}^{0}\left(\boldsymbol{x}^{1}, \boldsymbol{y}^{1}\right)}{D_{i}^{0}\left(\boldsymbol{x}^{1}, \boldsymbol{y}^{0}\right)} \frac{D_{i}^{0}\left(\boldsymbol{x}^{0}, \boldsymbol{y}^{1}\right)}{D_{i}^{0}\left(\boldsymbol{x}^{0}, \boldsymbol{y}^{0}\right)} \frac{D_{i}^{1}\left(\boldsymbol{x}^{1}, \boldsymbol{y}^{1}\right)}{D_{i}^{1}\left(\boldsymbol{x}^{1}, \boldsymbol{y}^{0}\right)} \frac{D_{i}^{1}\left(\boldsymbol{x}^{0}, \boldsymbol{y}^{1}\right)}{D_{i}^{1}\left(\boldsymbol{x}^{0}, \boldsymbol{y}^{0}\right)}\right)^{1 / 4} Q^{F}\left(\boldsymbol{p}^{1}, \boldsymbol{y}^{1}, \boldsymbol{p}^{0}, \boldsymbol{y}^{0}\right)\right] .}
\end{gathered}
$$

where $Q^{F}\left(\boldsymbol{p}^{1}, \boldsymbol{y}^{1}, \boldsymbol{p}^{0}, \boldsymbol{y}^{0}\right)$ and $Q^{F}\left(\boldsymbol{w}^{1}, \boldsymbol{x}^{1}, \boldsymbol{w}^{0}, \boldsymbol{x}^{0}\right)$ were defined after expression (49). The geometric mean of the base and comparison period $C C D$ indices can be replaced by the common technical efficiency change factor $E C_{i}\left(\boldsymbol{x}^{1}, \boldsymbol{y}^{1}, \boldsymbol{x}^{0}, \boldsymbol{y}^{0}\right)$ times the geometric mean of the technological change factors $\left[T C_{i}^{1,0}\left(\boldsymbol{x}^{1}, \boldsymbol{y}^{1}\right) \times T C_{i}^{1,0}\left(\boldsymbol{x}^{0}, \boldsymbol{y}^{0}\right)\right]^{1 / 2}$.

The complete input orientated decomposition of the Fisher PI can be calculated by the deatfprod $(X, Y, \ldots)$ function with the period parameter set to (geomean), the orientation parameter set to (io), and the decomp parameter set to (complete).

$>$ tfprod_io_geo_complete $=\operatorname{deatfprod}(X, Y, W, P$, 'orient', 'io', ...

$>$ tfpdisp(tfprod_io_geo_complete)

$$
\text { 'period', 'geomean', 'decomp', 'complete'); }
$$

Total Factor Productivity (TFP)

DMUs : 5

Inputs: 2 Outputs: 2 


\begin{tabular}{|c|c|c|c|c|c|}
\hline \multicolumn{6}{|c|}{$\begin{array}{l}\text { Price-weighted productivity index: } \\
\text { Reference period is Geometric mean }\end{array}$} \\
\hline DMUI & Prod I & $\mathrm{ECl}$ & $\mathrm{TCl}$ & IME | & SECI \\
\hline 11 & $0.4893 \mid$ & $0.6667 \mid$ & 1.00001 & $0.9337 \mid$ & 0.78611 \\
\hline 21 & $1.2663 \mid$ & 1.00001 & $1.1832 \mid$ & 0.96081 & $1.1138 \mid$ \\
\hline 31 & $1.3134 \mid$ & 1.00001 & 1.00001 & $1.2553 \mid$ & $1.0463 \mid$ \\
\hline 41 & $1.9787 \mid$ & 2.00001 & 1.00001 & $0.7687 \mid$ & 1.2870 \\
\hline 51 & $0.4607 \mid$ & $0.6667 \mid$ & $1.0328 \mid$ & $0.7883 \mid$ & $0.8487 \mid$ \\
\hline
\end{tabular}

Prod $=$ Fisher. EC = Efficiency Change $. T C=$ Technological Change. IME $=$ Input Mix Effect. SEC = Scale Effect.

The function returns the Fisher PI (PROD), the technical efficiency change factor (EC), the geometric mean of the technological change factors (TC), the geometric mean of the four input mix effects as presented in the third row of expression (52) (IME), and the geometric mean of the four scale effects as presented in the fourth row of the same expression (SEC). Each of the four separate effects in IME or SEC corresponds to a specific Path E, F, G or H, which can be recovered by running the previous base and comparison period decompositions. If distance function values are impossible to compute, the toolbox calculates the Fisher PI as the geometric mean of the Laspeyres and Paasche PIs.

The decomposition featuring the $C C D$ indices, as presented in (52), can be obtained by setting the decomp parameter to (ccd). The constant returns to scale specification is obtained by setting that parameter to (crs). This decomposition is valid if both technologies exhibit constant returns to scale, which can be tested by the deatestrtsm(X, Y, ...) function with the orient parameter set to (io).

\section{Share-Weighted Productivity Indices (Törnqvist)}

A share-weighted productivity index is defined as a weighted geometric mean of output quantity changes divided by a weighted geometric mean of input quantity changes. In particular, individual input quantity changes, $x_{n}^{1} / x_{n}^{0}$, and output quantity changes, $y_{m}^{1} / y_{m}^{0}$, are weighted by their respective cost and revenue shares in a certain period $t$. The generic definition is

$$
\operatorname{GPROD}\left(\boldsymbol{x}^{1}, \boldsymbol{y}^{1}, \boldsymbol{x}^{0}, \boldsymbol{y}^{0} ; \boldsymbol{s}^{t}, \boldsymbol{u}^{t}\right) \equiv \frac{\prod_{m=1}^{M}\left(y_{m}^{1} / y_{m}^{0}\right)^{u_{m}^{t}}}{\prod_{n=1}^{N}\left(x_{n}^{1} / x_{n}^{0}\right)^{s_{n}^{t}}}
$$

where $s_{n}^{t} \equiv w_{n}^{t} x_{n}^{t} / w^{t} \cdot x^{t}>0(n=1, \ldots, N), \sum_{n=1}^{N} s_{n}^{t}=1, u_{m}^{t} \equiv p_{m}^{t} y_{m}^{t} / p^{t} \cdot y^{t}>0(m=$ $1, \ldots, M), \sum_{m=1}^{M} u_{m}^{t}=1$. For $t=0$ the index is called GeoLaspeyres, and for $t=1$ the index is called GeoPaasche. The geometric mean of the two indices is the Törnqvist productivity 
index. This index can also be obtained by considering the 'average' viewpoint for the cost and revenue shares, $s^{0,1} \equiv\left(s^{0}+s^{1}\right) / 2$ and $\boldsymbol{u}^{0,1} \equiv\left(\boldsymbol{u}^{0}+\boldsymbol{u}^{1}\right) / 2$.

\subsection{The output orientated decomposition of a share-weighted productivity index}

The output orientated decomposition of the GeoLaspeyres PI

Following 'Path A', the GeoLaspeyres PI is decomposed as

$$
\begin{aligned}
G P R O D\left(\boldsymbol{x}^{1}, \boldsymbol{y}^{1}, \boldsymbol{x}^{0}, \boldsymbol{y}^{0} ; \boldsymbol{s}^{0}, \boldsymbol{u}^{0}\right) & =E C_{o}\left(\boldsymbol{x}^{1}, \boldsymbol{y}^{1}, \boldsymbol{x}^{0}, \boldsymbol{y}^{0}\right) \times T C_{o}^{1,0}\left(\boldsymbol{x}^{1}, \boldsymbol{y}^{1}\right) \times \\
& {\left[\frac{D_{o}^{0}\left(\boldsymbol{x}^{0}, \boldsymbol{y}^{0}\right)}{D_{o}^{0}\left(\boldsymbol{x}^{1}, \boldsymbol{y}^{0}\right)} \prod_{n=1}^{N}\left(x_{n}^{0} / x_{n}^{1}\right)^{s_{n}^{0}}\right] \times\left[\frac{D_{o}^{0}\left(\boldsymbol{x}^{1}, \boldsymbol{y}^{0}\right)}{D_{o}^{0}\left(\boldsymbol{x}^{1}, \boldsymbol{y}^{1}\right)} \prod_{m=1}^{M}\left(y_{m}^{1} / y_{m}^{0}\right)^{u_{m}^{0}}\right] . }
\end{aligned}
$$

The first two factors after the equality sign are the same as the first two factors of the Laspeyres PI in expression (43); i.e., technical efficiency change and technological change, respectively. The third factor captures the scale (including input mix) effect and the fourth factor measures the output mix effect.

Following the alternative 'Path B' delivers

$$
\begin{aligned}
G P R O D\left(\boldsymbol{x}^{1}, \boldsymbol{y}^{1}, \boldsymbol{x}^{0}, \boldsymbol{y}^{0} ; \boldsymbol{s}^{0}, \boldsymbol{u}^{0}\right) & =E C_{o}\left(\boldsymbol{x}^{1}, \boldsymbol{y}^{1}, \boldsymbol{x}^{0}, \boldsymbol{y}^{0}\right) \times T C_{o}^{1,0}\left(\boldsymbol{x}^{1}, \boldsymbol{y}^{1}\right) \times \\
& {\left[\frac{D_{o}^{0}\left(\boldsymbol{x}^{0}, \boldsymbol{y}^{1}\right)}{D_{o}^{0}\left(\boldsymbol{x}^{1}, \boldsymbol{y}^{1}\right)} \prod_{n=1}^{N}\left(x_{n}^{0} / x_{n}^{1}\right)^{s_{n}^{0}}\right] \times\left[\frac{D_{o}^{0}\left(\boldsymbol{x}^{0}, \boldsymbol{y}^{0}\right)}{D_{o}^{0}\left(\boldsymbol{x}^{0}, \boldsymbol{y}^{1}\right)} \prod_{m=1}^{M}\left(y_{m}^{1} / y_{m}^{0}\right)^{u_{m}^{0}}\right], }
\end{aligned}
$$

which differs from expression (54) in the scale effect and the output mix effect. Taking the geometric average of these two expressions yields

$$
\begin{gathered}
\operatorname{GPROD}\left(\boldsymbol{x}^{1}, \boldsymbol{y}^{1}, \boldsymbol{x}^{0}, \boldsymbol{y}^{0} ; \boldsymbol{s}^{0}, \boldsymbol{u}^{0}\right)=E C_{o}\left(\boldsymbol{x}^{1}, \boldsymbol{y}^{1}, \boldsymbol{x}^{0}, \boldsymbol{y}^{0}\right) \times T C_{o}^{1,0}\left(\boldsymbol{x}^{1}, \boldsymbol{y}^{1}\right) \times \\
{\left[\left(\frac{D_{o}^{0}\left(\boldsymbol{x}^{0}, \boldsymbol{y}^{0}\right)}{D_{o}^{0}\left(\boldsymbol{x}^{1}, \boldsymbol{y}^{0}\right)} \frac{D_{o}^{0}\left(\boldsymbol{x}^{0}, \boldsymbol{y}^{1}\right)}{D_{o}^{0}\left(\boldsymbol{x}^{1}, \boldsymbol{y}^{1}\right)}\right)^{1 / 2} \prod_{n=1}^{N}\left(x_{n}^{0} / x_{n}^{1}\right)^{s_{n}^{0}}\right] \times\left[\left(\frac{D_{o}^{0}\left(\boldsymbol{x}^{1}, \boldsymbol{y}^{0}\right)}{D_{o}^{0}\left(\boldsymbol{x}^{1}, \boldsymbol{y}^{1}\right)} \frac{D_{o}^{0}\left(\boldsymbol{x}^{0}, \boldsymbol{y}^{0}\right)}{D_{o}^{0}\left(\boldsymbol{x}^{0}, \boldsymbol{y}^{1}\right)}\right)^{1 / 2} \prod_{m=1}^{M}\left(y_{m}^{1} / y_{m}^{0}\right)^{u_{m}^{0}}\right]}
\end{gathered}
$$

The MATLAB function to calculate these decompositions is deatfgprod(X, Y, ...), with the orient parameter set to output orientation (oo) and the period parameter to (base). The decomposition parameter decomp is (complete).

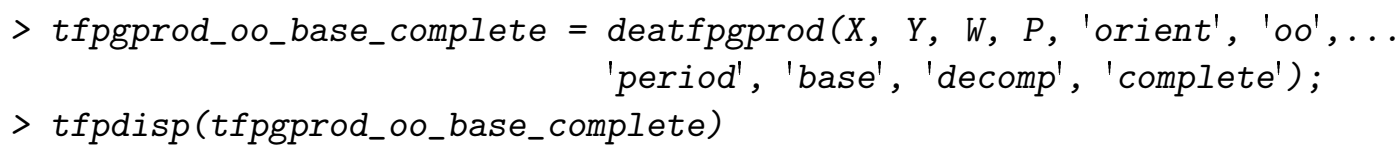

Total Factor Productivity (TFP) 
DMUs : 5

Inputs: 2 Outputs: 2

Model: tfp-gprod

Orientation: oo (Output orientated)

Returns to scale: vrs (Variable)

Share-weighted geometric productivity index:

Reference period is base period

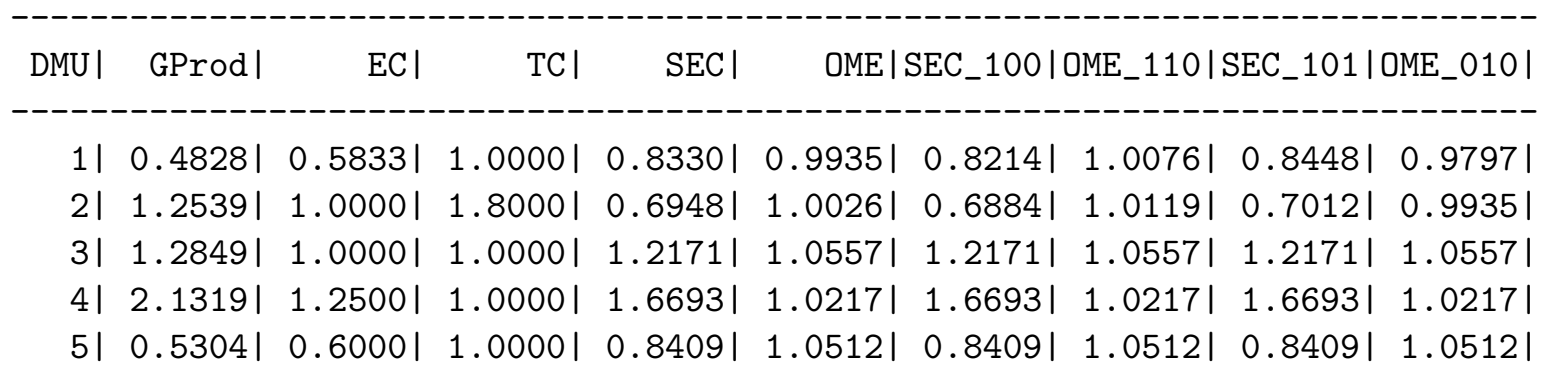

GProd = GeoLaspeyres. EC = Efficiency Change. $\mathrm{TC}=$ Technological Change.

$\mathrm{SEC}=$ Scale Effect. OME = Output Mix Effect.

The output of the function successively shows the GeoLaspeyres productivity index (GPROD), the technical efficiency change factor (EC), the technological change factor (TC), and the geometric means and component values of the scale effects and output mix effects corresponding to 'Path A' and 'Path B'. The geometric means are identified by SEC and OME. The components are identified by the time superscripts of the input and output arguments in each factor; e.g., SEC_100 represents the third factor in expression (54), counterpart to $S E C_{o}^{0}\left(\boldsymbol{x}^{1}, \boldsymbol{x}^{0}, \boldsymbol{y}^{0}\right)$ in expression (2). As calculation of some distance function values may be infeasible, the toolbox calculates the GeoLaspeyres PI directly from the data.

\section{Related decompositions of the GeoLaspeyres PI}

Recalling expression (6), expression (54) can be written as

$$
\begin{aligned}
\operatorname{GPROD}\left(\boldsymbol{x}^{1}, \boldsymbol{y}^{1}, \boldsymbol{x}^{0}, \boldsymbol{y}^{0} ; \boldsymbol{s}^{0}, \boldsymbol{u}^{0}\right) & =M_{o}^{0}\left(x^{1}, y^{1}, x^{0}, y^{0}\right) \times \\
& {\left[\frac{D_{o}^{0}\left(\boldsymbol{x}^{0}, \boldsymbol{y}^{0}\right)}{D_{o}^{0}\left(\boldsymbol{x}^{1}, \boldsymbol{y}^{0}\right)} \prod_{n=1}^{N}\left(x_{n}^{0} / x_{n}^{1}\right)^{s_{n}^{0}}\right] \times\left[\frac{D_{o}^{0}\left(\boldsymbol{x}^{1}, \boldsymbol{y}^{0}\right)}{D_{o}^{0}\left(\boldsymbol{x}^{1}, \boldsymbol{y}^{1}\right)} \prod_{m=1}^{M}\left(y_{m}^{1} / y_{m}^{0}\right)^{u_{m}^{0}}\right] . }
\end{aligned}
$$

Similar simplifications hold for expressions (55), and (56). To obtain these decompositions, the decomp parameter must be set to (ccd).

> tfpgprod_oo_base_ccd $=$ deatfpgprod $(X, Y, W, P$, 'orient', 'oo',.. 'period', 'base', 'decomp', 'ccd');

$>$ tfpdisp(tfpgprod_oo_base_ccd)

Total Factor Productivity (TFP) 
DMUs : 5

Inputs: 2 Outputs: 2

Model: tfp-gprod

Orientation: oo (Output orientated)

Returns to scale: vrs (Variable)

Share-weighted geometric productivity index:

Reference period is base period

\begin{tabular}{|c|c|c|c|c|c|c|c|c|}
\hline DMU I & GProd I & Mvrs | & SEC I & OME I & SEC_100I & OME_110| & SEC_101I & OME_010I \\
\hline & & & & & & & & \\
\hline 11 & 0.48281 & 0.58331 & 0.83301 & $0.9935 \mid$ & $0.8214 \mid$ & $1.0076 \mid$ & $0.8448 \mid$ & $0.9797 \mid$ \\
\hline 21 & $1.2539 \mid$ & 1.80001 & $0.6948 \mid$ & $1.0026 \mid$ & 0.68841 & $1.0119 \mid$ & 0.70121 & 0.99351 \\
\hline 31 & 1.2849 & 1.00001 & $1.2171 \mid$ & $1.0557 \mid$ & $1.2171 \mid$ & $1.0557 \mid$ & $1.2171 \mid$ & $1.0557 \mid$ \\
\hline 41 & $2.1319 \mid$ & 1.25001 & $1.6693 \mid$ & $1.0217 \mid$ & $1.6693 \mid$ & $1.0217 \mid$ & $1.6693 \mid$ & 1.0217 \\
\hline 51 & 0.53041 & 0.60001 & $0.8409 \mid$ & $1.0512 \mid$ & 0.84091 & $1.0512 \mid$ & $0.8409 \mid$ & 1.0512 \\
\hline
\end{tabular}

GProd = GeoLaspeyres. Mvrs = Malmquist VRS. SEC = Scale Effect.

$\mathrm{OME}=$ Output Mix Effect.

If the base period technology exhibits CRS (tested by the function deatestrtsm (X, Y, ...) with orient set to (oo)), setting the parameter decomp to (crs) delivers a table for the decomposition

$$
\begin{aligned}
& \operatorname{GPROD}\left(\boldsymbol{x}^{1}, \boldsymbol{y}^{1}, \boldsymbol{x}^{0}, \boldsymbol{y}^{0} ; \boldsymbol{s}^{0}, \boldsymbol{u}^{0}\right)=\check{M}_{o}^{0}\left(\boldsymbol{x}^{1}, \boldsymbol{y}^{1}, \boldsymbol{x}^{0}, \boldsymbol{y}^{0}\right) \times \\
& {\left[\frac{\check{D}_{o}^{0}\left(\boldsymbol{x}^{0}, \boldsymbol{y}^{0}\right)}{\check{D}_{o}^{0}\left(\boldsymbol{x}^{1}, \boldsymbol{y}^{0}\right)} \prod_{n=1}^{N}\left(x_{n}^{0} / x_{n}^{1}\right)^{s_{n}^{0}}\right] \times\left[\frac{\check{D}_{o}^{0}\left(\boldsymbol{x}^{1}, \boldsymbol{y}^{0}\right)}{\check{D}_{o}^{0}\left(\boldsymbol{x}^{1}, \boldsymbol{y}^{1}\right)} \prod_{m=1}^{M}\left(y_{m}^{1} / y_{m}^{0}\right)^{u_{m}^{0}}\right],}
\end{aligned}
$$

where the third factor measures the input mix effect and the fourth factor measures the output mix effect. The scale effect has vanished.

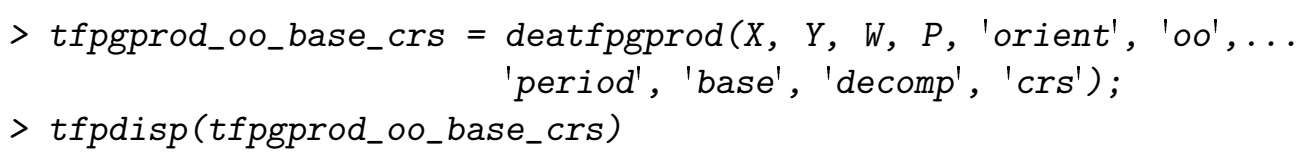

Total Factor Productivity (TFP)

DMUs : 5

Inputs: 2 Outputs: 2

Model: tfp-gprod

Orientation: oo (Output orientated)

Returns to scale: crs (Constant)

Share-weighted geometric productivity index: 
Reference period is base period

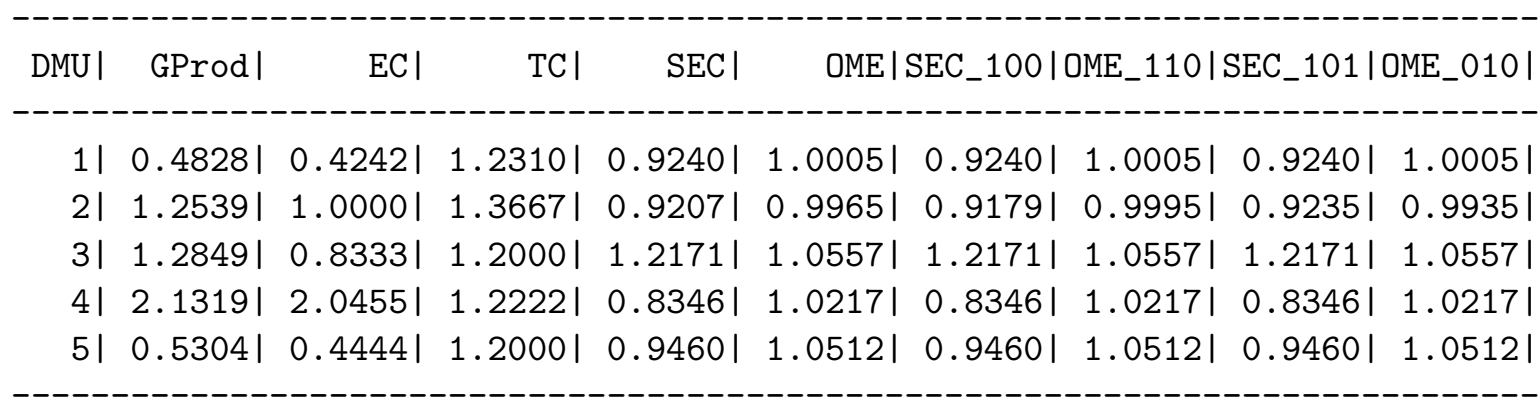

GProd $=$ GeoLaspeyres. $\mathrm{EC}=$ Efficiency Change. $\mathrm{TC}=$ Technological Change.

SEC = Scale Effect. OME = Output Mix Effect.

Under CRS the SEC factor measures only the input mix effect.

The output orientated decomposition of the GeoPaasche PI

Along 'Path C' and 'Path D' we obtain decompositions of the GeoPaasche PI. Their geometric mean, analogous to expression (56), reads

$$
\begin{gathered}
\operatorname{GPROD}\left(\boldsymbol{x}^{1}, \boldsymbol{y}^{1}, \boldsymbol{x}^{0}, \boldsymbol{y}^{0} ; \boldsymbol{s}^{1}, \boldsymbol{u}^{1}\right)=E C_{o}\left(\boldsymbol{x}^{1}, \boldsymbol{y}^{1}, \boldsymbol{x}^{0}, \boldsymbol{y}^{0}\right) \times T C_{o}^{1,0}\left(\boldsymbol{x}^{0}, \boldsymbol{y}^{0}\right) \times \\
{\left[\left(\frac{D_{o}^{1}\left(\boldsymbol{x}^{0}, \boldsymbol{y}^{0}\right)}{D_{o}^{1}\left(\boldsymbol{x}^{1}, \boldsymbol{y}^{0}\right)} \frac{D_{o}^{1}\left(\boldsymbol{x}^{0}, \boldsymbol{y}^{1}\right)}{D_{o}^{1}\left(\boldsymbol{x}^{1}, \boldsymbol{y}^{1}\right)}\right)^{1 / 2} \prod_{n=1}^{N}\left(x_{n}^{0} / x_{n}^{1}\right)^{s_{n}^{1}}\right] \times\left[\left(\frac{D_{o}^{1}\left(\boldsymbol{x}^{1}, \boldsymbol{y}^{0}\right)}{D_{o}^{1}\left(\boldsymbol{x}^{1}, \boldsymbol{y}^{1}\right)} \frac{D_{o}^{1}\left(\boldsymbol{x}^{0}, \boldsymbol{y}^{0}\right)}{D_{o}^{1}\left(\boldsymbol{x}^{0}, \boldsymbol{y}^{1}\right)}\right)^{1 / 2} \prod_{m=1}^{M}\left(y_{m}^{1} / y_{m}^{0}\right)^{u_{m}^{1}}\right] .}
\end{gathered}
$$

To obtain these decompositions, in the deatfgprod $(X, Y, \ldots)$ function the period parameter must be set to (comparison).

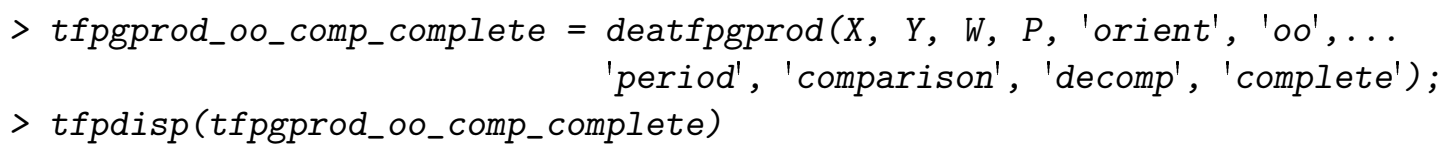

Total Factor Productivity (TFP)

DMUs : 5

Inputs: 2 Outputs: 2

Model: tfp-gprod

Orientation: oo (Output orientated)

Returns to scale: vrs (Variable)

Share-weighted geometric productivity index:

Reference period is comparison period 
DMU | GProd I ECI TC| SEC| OME ISEC_100|OME_110|SEC_101|OME_010 I

$1|0.4958| 0.5833|1.2222| 0.6859|1.0138| 0.6859|1.0138| 0.6859|1.0138|$

$2|1.2783| 1.0000|1.0000| 1.2801|0.9986| 1.2684|1.0078| 1.2919|0.9895|$

$3|1.3389| 1.0000|1.0000| 1.2910|1.0371| 1.2910|1.0371| 1.2910|1.0371|$

$4|1.8429| 1.2500|1.0000| 1.4210|1.0376| 1.4210|1.0376| 1.4210|1.0376|$

$5|0.3968| 0.6000|1.0000| 0.6417|1.0306| 0.6417|1.0306| 0.6417|1.0306|$

GProd $=$ GeoPaasche. $\mathrm{EC}=$ Efficiency Change. $\mathrm{TC}=$ Technological Change.

SEC = Scale Effect. OME = Output Mix Effect.

The first two factors can be aggregated to the output orientated, comparison period $C C D$ in$\operatorname{dex} M_{o}^{1}\left(x^{1}, y^{1}, x^{0}, y^{0}\right)$, as defined by expression (13). The corresponding table can be obtained by changing the decomp parameter from (complete) to (ccd). If the existence of constant returns to scale for the comparison period technology cannot be rejected, then the table is obtained by setting the decomp parameter to (crs).

\section{The output orientated decomposition of the Törnqvist PI}

Combining expressions (56) and (59) yields a decomposition of the Törnqvist productivity index, the share-weighted analogue of the Fisher productivity index,

$$
\begin{aligned}
& \operatorname{GPROD}\left(\boldsymbol{x}^{1}, \boldsymbol{y}^{1}, \boldsymbol{x}^{0}, \boldsymbol{y}^{0} ;\left(\boldsymbol{s}^{0}+\boldsymbol{s}^{1}\right) / 2,\left(\boldsymbol{u}^{0}+\boldsymbol{u}^{1}\right) / 2\right) \equiv \frac{Q^{T}\left(\boldsymbol{p}^{1}, \boldsymbol{y}^{1}, \boldsymbol{p}^{0}, \boldsymbol{y}^{0}\right)}{Q^{T}\left(\boldsymbol{w}^{1}, \boldsymbol{x}^{1}, \boldsymbol{w}^{0}, \boldsymbol{x}^{0}\right)}= \\
& {\left[M_{o}^{0}\left(\boldsymbol{x}^{1}, \boldsymbol{y}^{1}, \boldsymbol{x}^{0}, \boldsymbol{y}^{0}\right) M_{o}^{1}\left(\boldsymbol{x}^{1}, y^{1}, \boldsymbol{x}^{0}, \boldsymbol{y}^{0}\right)\right]^{1 / 2} \times} \\
& {\left[\left(\frac{D_{o}^{0}\left(\boldsymbol{x}^{0}, \boldsymbol{y}^{0}\right)}{D_{o}^{0}\left(\boldsymbol{x}^{1}, \boldsymbol{y}^{0}\right)} \frac{D_{o}^{0}\left(\boldsymbol{x}^{0}, \boldsymbol{y}^{1}\right)}{D_{o}^{0}\left(\boldsymbol{x}^{1}, \boldsymbol{y}^{1}\right)} \frac{D_{o}^{1}\left(\boldsymbol{x}^{0}, \boldsymbol{y}^{0}\right)}{D_{o}^{1}\left(\boldsymbol{x}^{1}, \boldsymbol{y}^{0}\right)} \frac{D_{o}^{1}\left(\boldsymbol{x}^{0}, \boldsymbol{y}^{1}\right)}{D_{o}^{1}\left(\boldsymbol{x}^{1}, \boldsymbol{y}^{1}\right)}\right)^{1 / 4} \frac{1}{Q^{T}\left(\boldsymbol{w}^{1}, \boldsymbol{x}^{1}, \boldsymbol{w}^{0}, \boldsymbol{x}^{0}\right)}\right] \times} \\
& {\left[\left(\frac{D_{o}^{0}\left(\boldsymbol{x}^{1}, \boldsymbol{y}^{0}\right)}{D_{o}^{0}\left(\boldsymbol{x}^{1}, \boldsymbol{y}^{1}\right)} \frac{D_{o}^{0}\left(\boldsymbol{x}^{0}, \boldsymbol{y}^{0}\right)}{D_{o}^{0}\left(\boldsymbol{x}^{0}, \boldsymbol{y}^{1}\right)} \frac{D_{o}^{1}\left(\boldsymbol{x}^{1}, \boldsymbol{y}^{0}\right)}{D_{o}^{1}\left(\boldsymbol{x}^{1}, \boldsymbol{y}^{1}\right)} \frac{D_{o}^{1}\left(\boldsymbol{x}^{0}, \boldsymbol{y}^{0}\right)}{D_{o}^{1}\left(\boldsymbol{x}^{0}, \boldsymbol{y}^{1}\right)}\right)^{1 / 4} Q^{T}\left(\boldsymbol{p}^{1}, \boldsymbol{y}^{1}, \boldsymbol{p}^{0}, \boldsymbol{y}^{0}\right)\right],}
\end{aligned}
$$

where $Q^{T}\left(p^{1}, y^{1}, p^{0}, y^{0}\right) \equiv \prod_{m=1}^{M}\left(y_{m}^{1} / y_{m}^{0}\right)^{\left(u_{m}^{0}+u_{m}^{1}\right) / 2}$ is the Törnqvist output quantity index and $Q^{T}\left(w^{1}, x^{1}, w^{0}, x^{0}\right) \equiv \prod_{n=1}^{N}\left(x_{n}^{1} / x_{n}^{0}\right)^{\left(s_{n}^{0}+s_{n}^{1}\right) / 2}$ the input quantity index. In this expression the geometric mean of the base and comparison period $C C D$ indices can be replaced by the common technical efficiency change factor $E C_{o}\left(\boldsymbol{x}^{1}, \boldsymbol{y}^{1}, \boldsymbol{x}^{0}, \boldsymbol{y}^{0}\right)$ multiplied by the geometric mean of the technological change factors, $\left[T C_{o}^{1,0}\left(\boldsymbol{x}^{1}, \boldsymbol{y}^{1}\right) \times T C_{o}^{1,0}\left(\boldsymbol{x}^{0}, \boldsymbol{y}^{0}\right)\right]^{1 / 2}$.

To calculate the output orientated decomposition of the Törnqvist PI by the deatfgprod(X, $\mathrm{Y}, \ldots$ ) function, the user sets period to (geomean), orientation to (oo), and decomp to (complete).

$>$ tfpgprod_oo_geo_complete $=\begin{array}{r}\text { deatfpgprod }(X, Y, W, P, \text { 'orient', 'oo',... } \\ \text { 'period', 'geomean', 'decomp', 'complete'); }\end{array}$
$>$ tfpdisp(tfpgprod_oo_geo_complete)

Total Factor Productivity (TFP) 


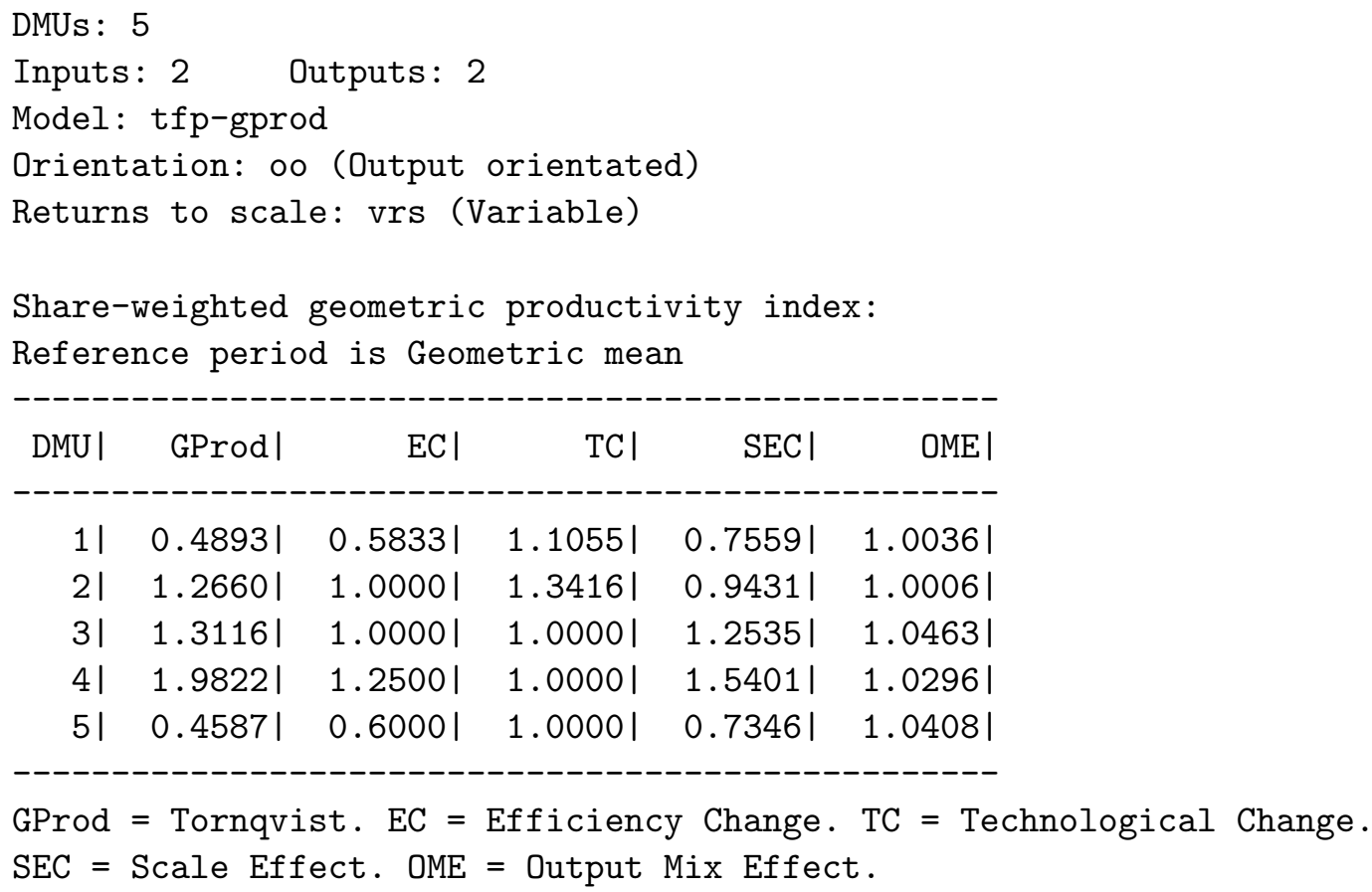

The output of the function successively shows the Törnqvist productivity index (GPROD), the technical efficiency change factor (EC), the geometric mean of the two technological change factors (TC), the geometric mean of the four component scale efficiency effects as presented in the third row of expression (60) (SEC), and the geometric mean of the four component output mix effects as presented in the fourth row of the same expression (OME). Each of these four components corresponds to a specific path $\mathrm{A}, \mathrm{B}, \mathrm{C}$ or $\mathrm{D}$, and can be recovered by running the previous base and comparison period decompositions. The toolbox calculates the Törnqvist index as the geometric mean of the GeoLaspeyres and GeoPaasche indices. Some factors might be missing because of infeasible distance function values.

The three-factor decomposition exhibiting the geometric mean of the two CCD indices, as shown in expression (60), is obtained by changing the decomp parameter to (ccd). If it can not be rejected that the technologies of both periods exhibit CRS, then the decompositions are obtained by setting the same parameter to (crs).

\subsection{The input orientated decomposition of a share-weighted productivity index}

All the share-weighted productivity indices discussed in the previous subsection can also be decomposed using input distance functions. The resulting decompositions are equivalent to those presented above.

\section{The input orientated decomposition of the GeoLaspeyres PI}

Along 'Path E' and 'Path F' one obtains expressions analogous to (54) and (55), but now using input distance functions. The geometric mean counterpart to expression (56) is 


$$
\begin{gathered}
G P R O D\left(\boldsymbol{x}^{1}, \boldsymbol{y}^{1}, \boldsymbol{x}^{0}, \boldsymbol{y}^{0} ; \boldsymbol{s}^{0}, \boldsymbol{u}^{0}\right)=E C_{i}\left(\boldsymbol{x}^{1}, \boldsymbol{y}^{1}, \boldsymbol{x}^{0}, \boldsymbol{y}^{0}\right) \times T C_{i}^{1,0}\left(\boldsymbol{x}^{1}, \boldsymbol{y}^{1}\right) \times \\
{\left[\left(\frac{D_{i}^{0}\left(\boldsymbol{x}^{1}, \boldsymbol{y}^{0}\right)}{D_{i}^{0}\left(\boldsymbol{x}^{0}, \boldsymbol{y}^{0}\right)} \frac{D_{i}^{0}\left(\boldsymbol{x}^{1}, \boldsymbol{y}^{1}\right)}{D_{i}^{0}\left(\boldsymbol{x}^{0}, \boldsymbol{y}^{1}\right)}\right)^{1 / 2} \prod_{n=1}^{N}\left(x_{n}^{0} / x_{n}^{1}\right)^{s_{n}^{0}}\right] \times\left[\left(\frac{D_{i}^{0}\left(\boldsymbol{x}^{1}, \boldsymbol{y}^{1}\right)}{D_{i}^{0}\left(\boldsymbol{x}^{1}, \boldsymbol{y}^{0}\right)} \frac{D_{i}^{0}\left(\boldsymbol{x}^{0}, \boldsymbol{y}^{1}\right)}{D_{i}^{0}\left(\boldsymbol{x}^{0}, \boldsymbol{y}^{0}\right)}\right)^{1 / 2} \prod_{m=1}^{M}\left(y_{m}^{1} / y_{m}^{0}\right)^{u_{m}^{0}}\right] .}
\end{gathered}
$$

The first two factors measure technical efficiency change and technological change, respectively. The third factor measures the input mix effect and the fourth factor measures the scale (including output mix) effect.

As before, in the syntax of the deatfgprod (X, Y, ...) function this decomposition requires setting the orient parameter to (io), the period parameter to (base), and the decomposition parameter decomp to (complete). The output of the function yields the GeoLaspeyres PI (GPROD), the technical efficiency change factor (EC), the technological change factor (TC). the geometric mean of the two input mix effect factors (IME), and the geometric mean of the scale (including output mix) effect factors (SEC). The components of each factor are identified by the time superscripts of the input and output arguments. For instance, IME_100 identifies the first half of the third factor in expression (61), that is,

$$
\frac{D_{i}^{0}\left(\boldsymbol{x}^{1}, \boldsymbol{y}^{0}\right)}{D_{i}^{0}\left(\boldsymbol{x}^{0}, \boldsymbol{y}^{0}\right)} \prod_{n=1}^{N}\left(x_{n}^{0} / x_{n}^{1}\right)^{s_{n}^{0}}
$$

This is the counterpart to $I M E^{0}\left(\boldsymbol{x}^{1}, \boldsymbol{x}^{0}, \boldsymbol{y}^{0}\right)$ in expression (17). In some cases the distance function might be infeasible, and therefore some of the factors involved in the decompositions cannot be presented. Then the toolbox calculates the GeoLaspeyres PI directly from the data.

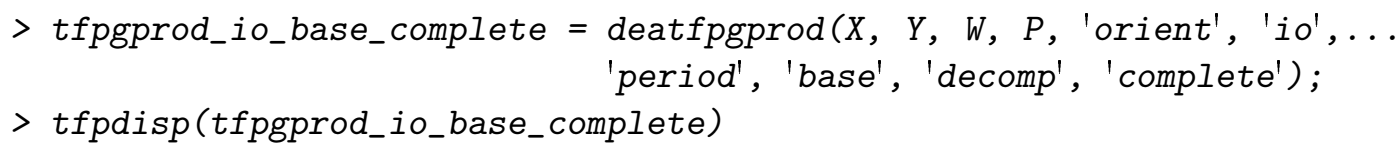

Total Factor Productivity (TFP)

DMUs : 5

Inputs: 2 Outputs: 2

Model: tfp-gprod

Orientation: io (Input orientated)

Returns to scale: vrs (Variable)

Share-weighted geometric productivity index:

Reference period is base period

DMU | GProd I EC| TC| IME | SEC|IME_100|SEC_110|IME_101|SEC_010|


$1|0.4828| 0.6667|1.0000| 0.9240|0.7837| 0.9240|0.7837| 0.9240|0.7837|$

$2|1.2539| 1.0000|1.4000| 0.9261|0.9671| 0.9179|0.9757| 0.9343|0.9586|$

$3|1.2849| 1.0000|1.0000| 1.2171|1.0557| 1.2171|1.0557| 1.2171|1.0557|$

$4|2.1319| 2.0000|1.0000| 0.8346|1.2772| 0.8346|1.2772| 0.8346|1.2772|$

$5|0.5304| 0.6667|1.0667| 0.9057|0.8234| 0.9460|0.7884| 0.8672|0.8601|$

GProd $=$ GeoLaspeyres. EC $=$ Efficiency Change. $\mathrm{TC}=$ Technological Change. IME $=$ Input Mix Effect. SEC = Scale Effect.

To recover the corresponding input orientated CCD index $M_{i}^{0}\left(\boldsymbol{x}^{1}, \boldsymbol{y}^{1}, \boldsymbol{x}^{0}, \boldsymbol{y}^{0}\right)$, the decomp parameter must be set to (ccd). If the base period technology exhibits CRS (which is checked by running the function deatestrtsm (X, Y, ...) with orient set to (io)), then setting the decomp parameter to (crs) calculates the appropriate decomposition. In this case, the fourth factor captures only the output mix effect, as scale effects do not play any role under CRS.

The input orientated decomposition of the GeoPaasche PI

The comparison period analogue of expression (61), corresponding to the geometric mean of 'Path G' and 'Path H', is

$$
\begin{gathered}
G P R O D\left(\boldsymbol{x}^{1}, \boldsymbol{y}^{1}, \boldsymbol{x}^{0}, \boldsymbol{y}^{0} ; \boldsymbol{s}^{1}, \boldsymbol{u}^{1}\right)=E C_{i}\left(\boldsymbol{x}^{1}, \boldsymbol{y}^{1}, \boldsymbol{x}^{0}, \boldsymbol{y}^{0}\right) \times T C_{i}^{1,0}\left(\boldsymbol{x}^{0}, \boldsymbol{y}^{0}\right) \times \\
{\left[\left(\frac{D_{i}^{1}\left(\boldsymbol{x}^{1}, \boldsymbol{y}^{0}\right)}{D_{i}^{1}\left(\boldsymbol{x}^{0}, \boldsymbol{y}^{0}\right)} \frac{D_{i}^{1}\left(\boldsymbol{x}^{1}, \boldsymbol{y}^{1}\right)}{D_{i}^{1}\left(\boldsymbol{x}^{0}, \boldsymbol{y}^{1}\right)}\right)^{1 / 2} \prod_{n=1}^{N}\left(x_{n}^{0} / x_{n}^{1}\right)^{s_{n}^{1}}\right] \times\left[\left(\frac{D_{i}^{1}\left(\boldsymbol{x}^{1}, \boldsymbol{y}^{1}\right)}{D_{i}^{1}\left(\boldsymbol{x}^{1}, \boldsymbol{y}^{0}\right)} \frac{D_{i}^{1}\left(\boldsymbol{x}^{0}, \boldsymbol{y}^{1}\right)}{D_{i}^{1}\left(\boldsymbol{x}^{0}, \boldsymbol{y}^{0}\right)}\right)^{1 / 2} \prod_{m=1}^{M}\left(y_{m}^{1} / y_{m}^{0}\right)^{u_{m}^{1}}\right] .}
\end{gathered}
$$

This decomposition can be computed by setting the period parameter to (comparison). The decomposition featuring the comparison period CCD index and the decomposition when CRS (of the comparison period technology) holds, require setting the decomp parameter to (ccd) and (crs), respectively.

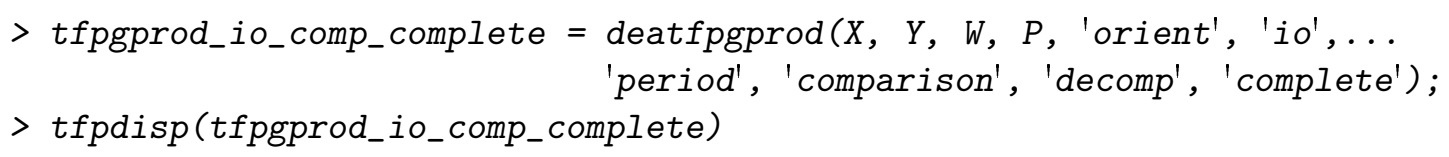

Total Factor Productivity (TFP)

DMUs : 5

Inputs: 2 Outputs: 2

Model: tfp-gprod

Orientation: io (Input orientated)

Returns to scale: vrs (Variable)

Share-weighted geometric productivity index:

Reference period is comparison period 


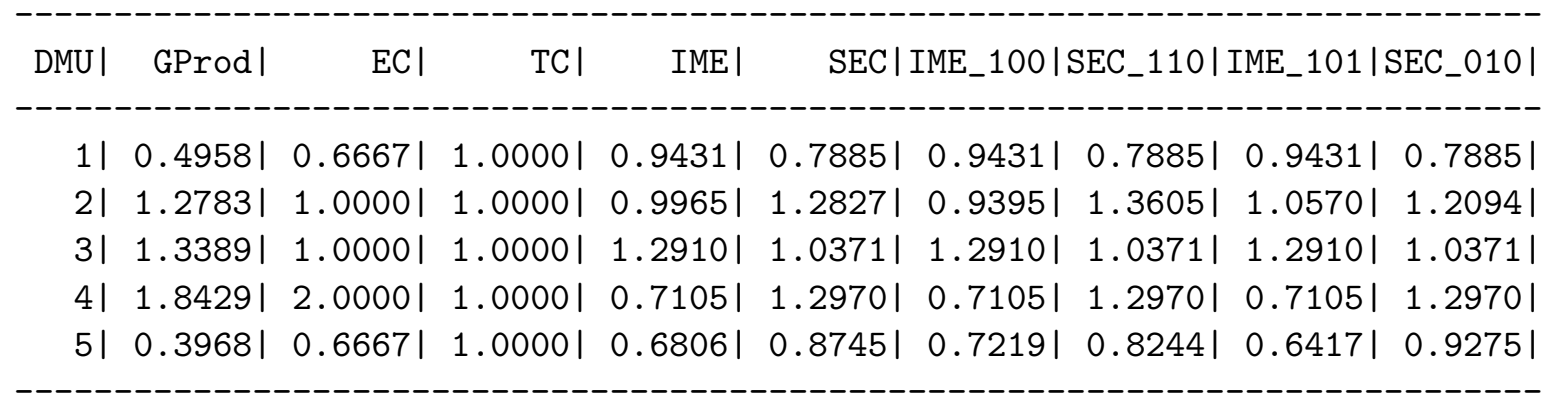

GProd $=$ GeoPaasche. EC = Efficiency Change. $\mathrm{TC}=$ Technological Change. IME $=$ Input Mix Effect. SEC = Scale Effect.

\section{The input orientated decomposition of the Törnqvist PI}

By taking the geometric mean of expressions (61) and (62), one obtains the input orientated decomposition of the Törnqvist productivity index,

$$
\begin{aligned}
& \operatorname{GPROD}\left(\boldsymbol{x}^{1}, \boldsymbol{y}^{1}, \boldsymbol{x}^{0}, \boldsymbol{y}^{0} ; \boldsymbol{w}^{1}, \boldsymbol{p}^{1},\left(\boldsymbol{s}^{0}+\boldsymbol{s}^{1}\right) / 2,\left(\boldsymbol{u}^{0}+\boldsymbol{u}^{1}\right) / 2\right) \equiv \frac{Q^{T}\left(\boldsymbol{p}^{1}, \boldsymbol{y}^{1}, \boldsymbol{p}^{0}, \boldsymbol{y}^{0}\right)}{Q^{T}\left(\boldsymbol{w}^{1}, \boldsymbol{x}^{1}, \boldsymbol{w}^{0}, \boldsymbol{x}^{0}\right)}= \\
& {\left[M_{i}^{0}\left(\boldsymbol{x}^{1}, \boldsymbol{y}^{1}, \boldsymbol{x}^{0}, \boldsymbol{y}^{0}\right) M_{i}^{1}\left(\boldsymbol{x}^{1}, y^{1}, \boldsymbol{x}^{0}, \boldsymbol{y}^{0}\right)\right]^{1 / 2} \times} \\
& {\left[\left(\frac{D_{i}^{0}\left(\boldsymbol{x}^{1}, \boldsymbol{y}^{0}\right)}{D_{i}^{0}\left(\boldsymbol{x}^{0}, \boldsymbol{y}^{0}\right)} \frac{D_{i}^{0}\left(\boldsymbol{x}^{1}, \boldsymbol{y}^{1}\right)}{D_{i}^{0}\left(\boldsymbol{x}^{0}, \boldsymbol{y}^{1}\right)} \frac{D_{i}^{1}\left(\boldsymbol{x}^{1}, \boldsymbol{y}^{0}\right)}{D_{i}^{1}\left(\boldsymbol{x}^{0}, \boldsymbol{y}^{0}\right)} \frac{D_{i}^{1}\left(\boldsymbol{x}^{1}, \boldsymbol{y}^{1}\right)}{D_{i}^{1}\left(\boldsymbol{x}^{0}, \boldsymbol{y}^{1}\right)}\right)^{1 / 4} \frac{1}{Q^{T}\left(\boldsymbol{w}^{1}, \boldsymbol{x}^{1}, \boldsymbol{w}^{0}, \boldsymbol{x}^{0}\right)}\right] \times} \\
& {\left[\left(\frac{D_{i}^{0}\left(\boldsymbol{x}^{1}, \boldsymbol{y}^{1}\right)}{D_{i}^{0}\left(\boldsymbol{x}^{1}, \boldsymbol{y}^{0}\right)} \frac{D_{i}^{0}\left(\boldsymbol{x}^{0}, \boldsymbol{y}^{1}\right)}{D_{i}^{0}\left(\boldsymbol{x}^{0}, \boldsymbol{y}^{0}\right)} \frac{D_{i}^{1}\left(\boldsymbol{x}^{1}, \boldsymbol{y}^{1}\right)}{D_{i}^{1}\left(\boldsymbol{x}^{1}, \boldsymbol{y}^{0}\right)} \frac{D_{i}^{1}\left(\boldsymbol{x}^{0}, \boldsymbol{y}^{1}\right)}{D_{i}^{1}\left(\boldsymbol{x}^{0}, \boldsymbol{y}^{0}\right)}\right)^{1 / 4} Q^{T}\left(\boldsymbol{p}^{1}, \boldsymbol{y}^{1}, \boldsymbol{p}^{0}, \boldsymbol{y}^{0}\right)\right] .}
\end{aligned}
$$

where $Q^{T}\left(p^{1}, y^{1}, p^{0}, y^{0}\right)$ and $\left.Q^{T}\left(w^{1}, x^{1}, w^{0}, x^{0}\right)\right)$ are defined as in (60). To obtain the fourfactor decomposition, the geometric mean of the base and comparison period $C C D$ indices must be replaced by the common technical efficiency change factor $E C_{i}\left(\boldsymbol{x}^{1}, \boldsymbol{y}^{1}, \boldsymbol{x}^{0}, \boldsymbol{y}^{0}\right)$ times the geometric mean of two technological change factors, $\left[T C_{i}^{1,0}\left(\boldsymbol{x}^{1}, \boldsymbol{y}^{1}\right) \times T C_{i}^{1,0}\left(\boldsymbol{x}^{0}, \boldsymbol{y}^{0}\right)\right]^{1 / 2}$.

This decomposition is obtained from the deatfprod(X, Y, ...) function if the period parameter is set to (geomean), the orientation parameter to (io), and the decomp parameter to (complete).

The output of the function returns the Törnqvist PI (GPROD) the technical efficiency change factor(EC), the geometric mean of the two technological change factors (TC), the geometric mean of the four component input mix effects as presented in the third row of expression (63) (IME), and the geometric mean of the four component scale (including output mix) effects as presented in the fourth row of the same expression (SEC). Each component of IME and SEC corresponds to a specific path E, F, G or H, and can be recovered by running the previous base and comparison period decompositions. In the case of infeasibilities the toolbox reports the Törnqvist PI directly from the data.

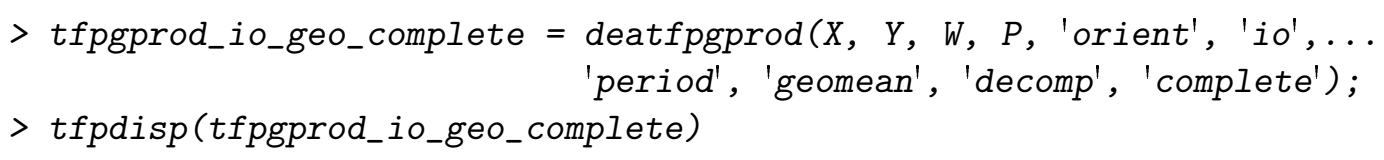




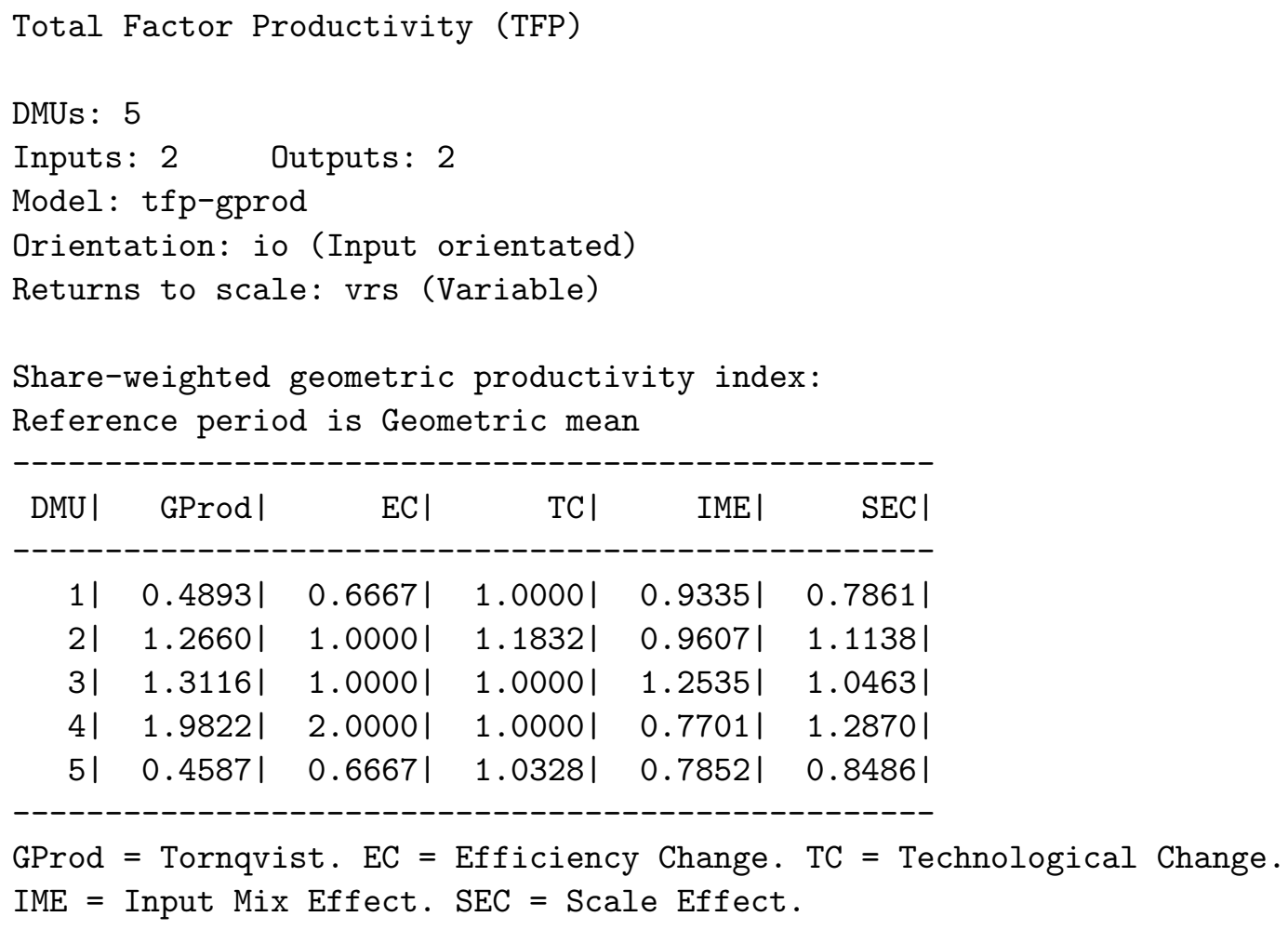

Finally, the three-factor decomposition featuring the CCD indices, expression (63), is obtained by setting the decomp parameter to (ccd). The CRS decomposition is obtained by setting this parameter to (crs). This decomposition should be used if the existence of CRS for both technologies has been statistically confirmed by running the deatestrtsm $(\mathrm{X}, \mathrm{Y}, \ldots)$ function, with the orient parameter set to (io).

\section{Advanced options, displaying and exporting results}

\subsection{Specifying DMU names}

By default, observations (DMUs) are numbered from 1 to $K$ t. However, custom names can be assigned to DMUs by adding the parameter names and a cell string with the desired names when calling the functions. Using data from the example:

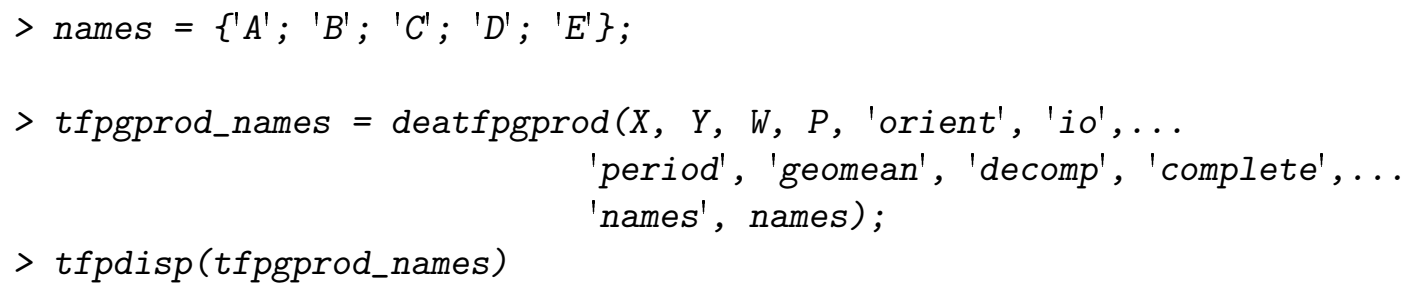

Total Factor Productivity (TFP) 


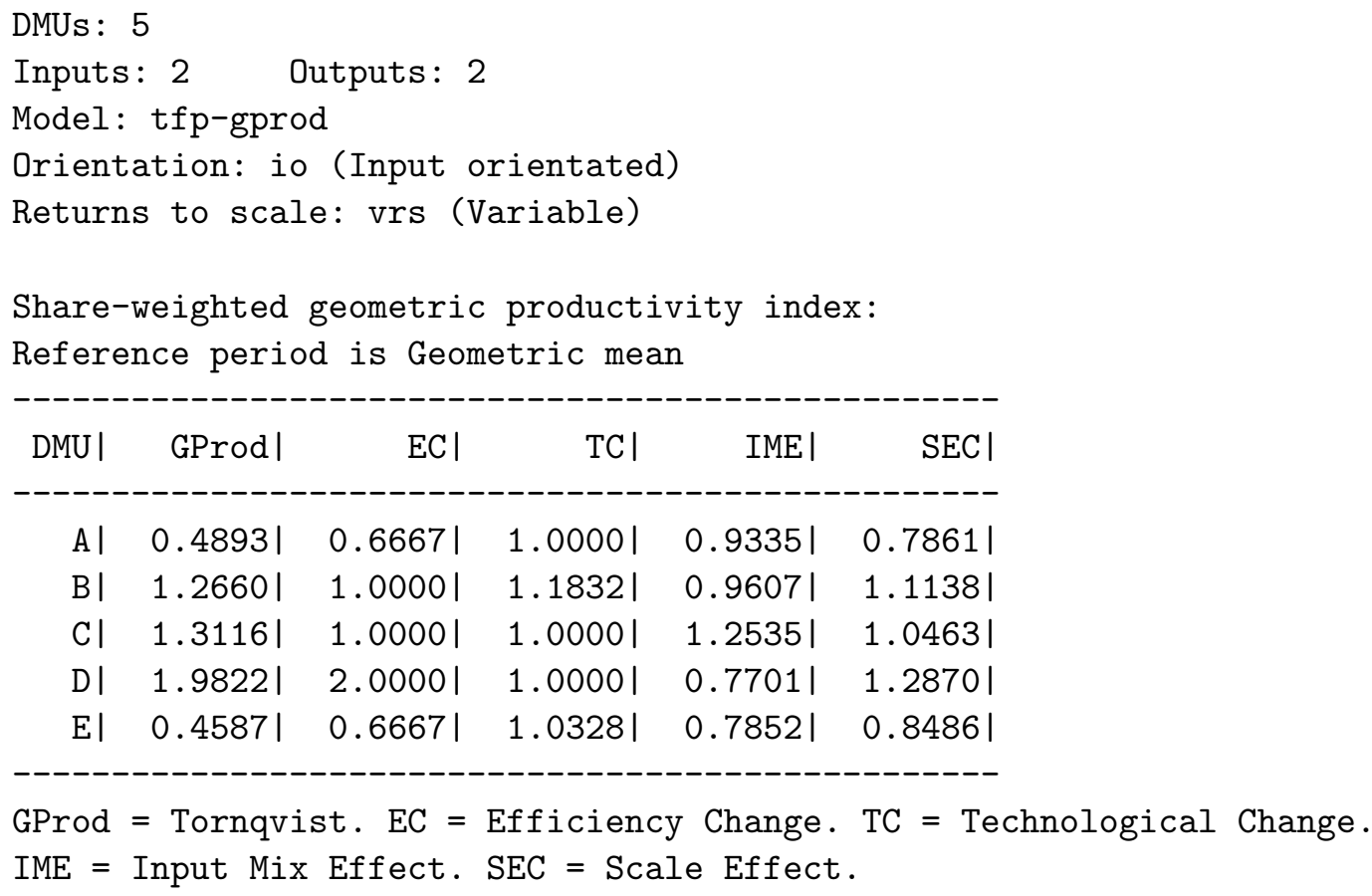

$\begin{array}{rrrrrr}\text { DMU | } & \text { GProd | } & \text { EC | } & \text { TC | } & \text { IME | } & \text { SEC | } \\ \text { A I } & 0.4893 \mid & 0.6667 \mid & 1.0000 \mid & 0.9335 \mid & 0.7861 \mid \\ \text { B I } & 1.2660 \mid & 1.0000 \mid & 1.1832 \mid & 0.9607 \mid & 1.1138 \mid \\ \text { C I } & 1.3116 \mid & 1.0000 \mid & 1.0000 \mid & 1.2535 \mid & 1.0463 \mid \\ \text { D I } & 1.9822 \mid & 2.0000 \mid & 1.0000 \mid & 0.7701 \mid & 1.2870 \mid \\ \text { E I } & 0.4587 \mid & 0.6667 \mid & 1.0328 \mid & 0.7852 \mid & 0.8486 \mid\end{array}$

GProd = Tornqvist. EC = Efficiency Change. $\mathrm{TC}=$ Technological Change. IME = Input Mix Effect. SEC = Scale Effect.

Another approach is to change the field names of the returned tfpout structure before displaying or exporting the results.

\subsection{Custom display}

When calling the tfpdisp (out, dispstr) function after computing a certain decomposition, appropriate information concerning the estimated model will be displayed on the screen. This setting can be changed to display bespoke information by specifying in the tfpdisp function the string dispstr (display string) as a second parameter.

For example, the default dispstr after using the deatfpgrpod function with the geometric mean option is names/tfp. GProd/tfp.EC/tfp.TC/tfp. IME/tfp. SEC. The fields displayed in the output table must be separated by a / and include the names corresponding to the field names of the tfpout structure. The available fields are presented in Table 1.

To display only the names of the DMUs and their productivity index numbers, dispstr must be specified as names/tfp. GProd.

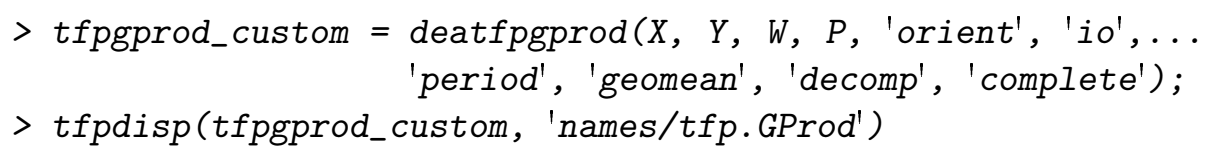

Total Factor Productivity (TFP)

DMUs : 5 


\begin{tabular}{l|l}
\hline \multicolumn{2}{c}{ Common fields } \\
\hline Field & Data \\
names & DMU names \\
TC & Technical efficiency change \\
\hline \multicolumn{1}{c}{ Technological change } \\
\hline tfp.M & \multicolumn{1}{c}{ Productivity indices } \\
tfp.MB & Moorsteen-Bjurek productivity index (after deatfpmb) \\
tfp.Prod & Price-weighted productivity index (after deatfprod) \\
tfp.GProd & Share-weighted productivity index (after deatfpgprod) \\
\hline \multicolumn{1}{|c}{ Output orientation } \\
\hline tfp.SEC & Scale effect (geometric mean) \\
tfp.OME & Output mix effect (geometric mean) \\
tfp.SEC_100 & $S E C_{o}^{t}\left(\boldsymbol{x}^{1}, \boldsymbol{x}^{0}, \boldsymbol{y}^{0}\right)$ \\
tfp.OME_110 & $O M E^{t}\left(\boldsymbol{x}^{1}, \boldsymbol{y}^{1}, \boldsymbol{y}^{0}\right)$ \\
tfp.SEC_101 & $S E C_{o}^{t}\left(\boldsymbol{x}^{1}, \boldsymbol{x}^{0}, \boldsymbol{y}^{1}\right)$ \\
tfp.OME_010 & $O M E^{t}\left(\boldsymbol{x}^{0}, \boldsymbol{y}^{1}, \boldsymbol{y}^{0}\right)$ \\
\hline \multicolumn{2}{|c}{ Input orientation } \\
\hline tfp.SEC & Scale effect (geometric mean) \\
tfp.IME & Input mix effect (geometric mean) \\
tfp.IME_100 & $I M E^{t}\left(\boldsymbol{x}^{1}, \boldsymbol{x}^{0}, \boldsymbol{y}^{0}\right)$ \\
tfp.SEC_110 & $S E C_{i}^{t}\left(\boldsymbol{x}^{1}, \boldsymbol{y}^{1}, \boldsymbol{y}^{0}\right)$ \\
tfp.IME_101 & $I M E^{t}\left(\boldsymbol{x}^{1}, \boldsymbol{y}^{0}, \boldsymbol{y}^{1}\right)$ \\
tfp.SEC_010 & $S E C_{i}^{t}\left(\boldsymbol{x}^{0}, \boldsymbol{x}^{1}, \boldsymbol{y}^{0}\right)$ \\
\hline
\end{tabular}

Table 1: Fields of the tfpout structure available for the dispstr string. 


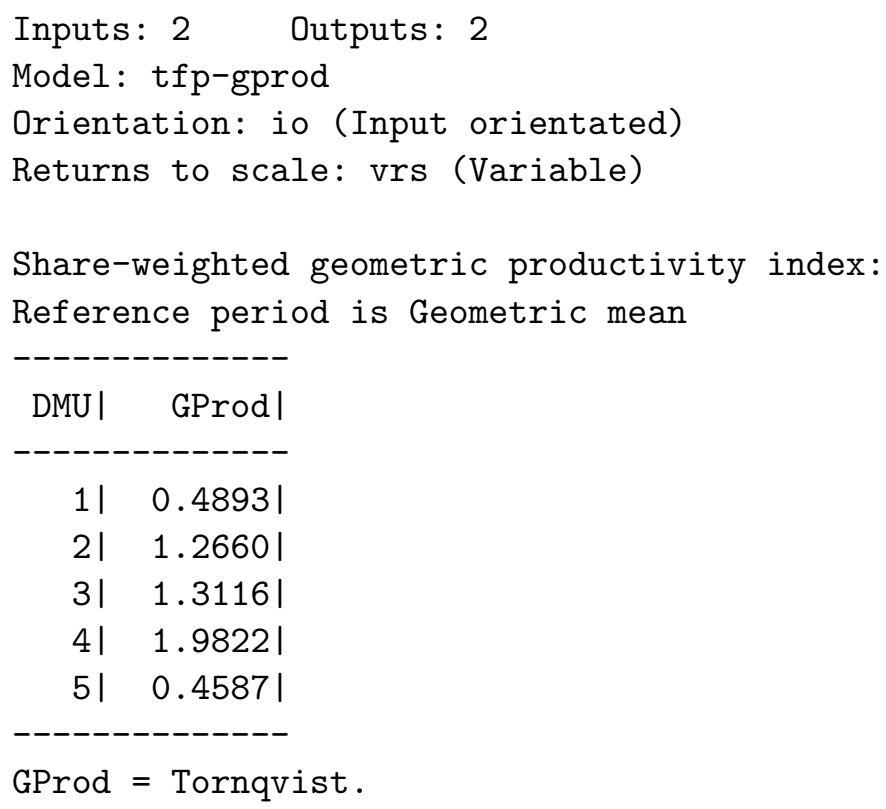

\subsection{Exporting results}

Results can easily be exported to various file formats for posterior analysis and sharing. First, the tfpout structure should be converted to a MATLAB table data type by using the tfp2table(out, dispstr) function with the desired dispstr. ${ }^{6}$

$>$ tfpgprod_exp $=$ deatfpgprod $(X, Y, W, P$, 'orient', 'io',... 'period', 'geomean', 'decomp', 'complete');

$>T=t f p 2 t a b l e\left(t f p g p r o d \_\exp \right)$;

Then, the table can be exported by using the MATLAB function writetable. ${ }^{7}$

> writetable(T, 'tfpgprod_results.csv');

\section{Conclusion}

The new Total Factor Productivity Toolbox allows the calculation and decomposition of the most popular productivity indices in an organized environment. The concept of distance function, implemented through non-parametric Data Envelopment Analysis, is the building block for the various decompositions. If only quantities are available, the toolbox calculates and decomposes TFP change according to the Malmquist and Moorsteen-Bjurek definitions. If also prices are available, classical TFP indices such as Fisher and Törnqvist can be calculated and decomposed.

\footnotetext{
${ }^{6}$ If the dispstr parameter is omitted, the default is used.

${ }^{7}$ See the official MATLAB documentation for this function at http://www.mathworks.com/help/matlab/ ref/writetable.html.
} 
By applying the toolbox, complex and lengthy decompositions involving panel data are rendered operational in a systematic way. It allows for many options concerning the orientation, reference period, variable or constant returns to scale, and preferred TFP definition and decomposition, so as to identify the contribution that different factors make to productivity change. The nature of returns to scale, either constant or variable, can also be tested by bootstrapping techniques. This allows researchers to set the model parameters to CRS, if its existence cannot be statistically rejected.

We showed how to organize the data, use the available functions, and interpret the results. To illustrate the toolbox we calculated all the indices with a common example so that alternative results can easily be compared. The new toolbox is a valid self-contained MATLAB package for the measurement and decomposition of TFP change.

Since the code is freely available in an open source repository on GitHub, under the GNU General Public License version 3, users will benefit from the collaboration and review of the community, and can check and modify the code to adapt it to their own needs and extend it to new definitions. ${ }^{8}$

\section{Acknowledgments}

José L. Zofío acknowledges support from the Spanish Ministry for Economy and Competitiveness under grants ECO2016-79650-P and MTM2016-79765-P. The views expressed in this paper are those of the authors and may not in any circumstances be regarded as stating an official position of the European Commission.

\section{References}

Balk BM (1998). Industrial Price, Quantity, and Productivity Indices: The MicroEconomic Theory and an Application. Springer US. URL http://doi.org/10.1007/ 978-1-4757-5454-4.

Balk BM (2001). "Scale Efficiency and Productivity Change." Journal of Productivity Analysis, 15(3), 159-183. ISSN 1573-0441. doi:10.1023/A:1011117324278.

Balk BM (2003). "The Residual: On Monitoring and Benchmarking Firms, Industries, and Economies with Respect to Productivity." Journal of Productivity Analysis, 20(1), 5-47. ISSN 1573-0441. doi:10.1023/A:1024817024364.

Balk BM, Zofío JL (2018). "The Many Decompositions of Total Factor Productivity Change." No. ERS-2018-003-LIS. ERIM Report Series Research in Management. Erasmus Research Institute of Management. Erasmus University, URL http://hdl.handle. net/1765/104721.

Banker R, Charnes A, Cooper W (1984). "Some Models for Estimating Technical and Scale Inefficiencies in Data Envelopment Analysis." Management Science, 30(9), 1078-1092. doi:https://doi.org/10.1287/mnsc.30.9.1078.

\footnotetext{
${ }^{8}$ The address of the repository is https://github.com/joselzofio/TFPMATLAB.
} 
Bogetoft P, Otto L (2011). Benchmarking with DEA, SFA, and R. Springer-Verlag, New York. doi:http://doi.org/10.1007/978-1-4419-7961-2.

Caves DW, Christensen LR, Diewert WE (1982). "The Economic Theory of Index Numbers and the Measurement of Input, Output, and Productivity." Econometrica, 50(6), 13931414. doi: 10.2307/1913388.

Charnes A, Cooper W, Rhodes E (1978). "Measuring the Efficiency of Decision Making Units." European Journal of Operational Research, 2(6), 429 - 444. ISSN 0377-2217. doi: 10.1016/0377-2217(78)90138-8.

Coelli T (1998). "A Guide to DEAP V2.1: A Data Envelopment Analysis (Computer) Program." CEPA Working Paper 96/08. Centre for Efficiency and Productivity Analysis, University of Queensland, URL http://www.uq.edu.au/economics/cepa/deap.php.

Coelli T (1999). "TFPIP V1: Total Factor Productivity Index (Computer) Program." Centre for Efficiency and Productivity Analysis, University of Queensland, URL http://www.uq. edu.au/economics/cepa/tfpip.php.

Cooper WW, Seiford LM, Tone K (2007). Data Envelopment Analysis: A Comprehensive Text with Models, Applications, References and DEA-Solver Software. NJ: Princeton University Press., Princeton. doi:10.1007/978-0-387-45283-8.

Daraio C, Kerstens K, Nepomuceno T, Sickles RC (2017). "Productivity and Efficiency Analysis Software: An Exploratory Bibliographical Survey of the Options." WP No. 2017-EQM05. IESEG School of Management, Lille, France.

Diewert WE, Fox KJ (2017). "Decomposing Productivity Indexes into Explanatory Factors." European Journal of Operational Research, 256(1), 275 - 291. ISSN 0377-2217. doi: https://doi.org/10.1016/j.ejor.2016.05.043.

Econometric Software I (2014). LIMDEP11. Plainview, NY. URL http://www.limdep.com/.

Emrouznejad A, Thanassoulis E (2011). "Performance Improvement Management Software: PIM-DEAsoft-V3.0 User Guide." Performance Improvement Management Ltd, Coventry, U.K., URL http://www. deasoftware.co.uk/.

Färe R, Grosskopf S, Lindgren B, Roos P (1994). "Productivity Developments in Swedish Hospitals: A Malmquist Output Index Approach." In A Charnes, WW Cooper, AY Lewin, LM Seiford (eds.), Data Envelopment Analysis: Theory, Methodology, and Applications, pp. 253-272. Springer Netherlands, Dordrecht. doi:10.1007/978-94-011-0637-5_13.

Färe R, Grosskopf S, Margaritis D (2008). "Efficiency and Productivity: Malmquist and More." In HO Fried, CAK Lovell, SS Schmidt (eds.), The Measurement of Productive Efficiency and Productivity Change, pp. 522-621. Oxford University Press, New York. doi: 10.1093/acprof : oso/9780195183528.003.0005.

Färe R, Grosskopf S, Norris M, Zhang Z (1994). "Productivity Growth, Technical Progress, and Efficiency Change in Industrialized Countries." The American Economic Review, 84(1), 66-83. URL http://www.jstor.org/stable/2951340. 
Färe R, Primont D (1995). Multi-Output Production and Duality: Theory and Applications. Springer Netherlands. doi:10.1007/978-94-011-0651-1.

Grifell-Tatjé E, Lovell C (1995). "A Note on the Malmquist Productivity Index." Economics Letters, 47(2), 169 - 175. ISSN 0165-1765. doi:https://doi.org/10.1016/ 0165-1765 (94) 00497-P.

Lee C, Lee KR, Lim BI (2011). "Malmquist Productivity Analysis using DEA Frontier in Stata." Korea National Defense University, Seoul, South Korea., URL https://ideas. repec.org/p/boc/chic11/21.html.

Lovell CAK (2003). "The Decomposition of Malmquist Productivity Indexes." Journal of Productivity Analysis, 20(3), 437-458. ISSN 1573-0441. doi:10.1023/A:1027312102834.

Moorsteen RH (1961). "On Measuring Productive Potential and Relative Efficiency." The Quarterly Journal of Economics, 75(3), 451-467. doi:10.2307/1885133.

O'Donnell (2011). "DPIN 3.0: A Program for Decomposing Productivity Index Numbers." Centre for Efficiency and Productivity Analysis, University of Queensland, URL http: //www.uq.edu.au/economics/cepa/dpin.php.

O’Donnell CJ (2012). "An Aggregate Quantity Framework for Measuring and Decomposing Productivity Change." Journal of Productivity Analysis, 38(3), 255-272. ISSN 1573-0441. doi:10.1007/s11123-012-0275-1.

O’Donnell CJ (2014). "Econometric Estimation of Distance Functions and Associated Measures of Productivity and Efficiency Change." Journal of Productivity Analysis, 41(2), 187-200. ISSN 1573-0441. doi:10.1007/s11123-012-0311-1.

OECD (2001). Measuring Productivity. Measurement of Aggregate and Industry Level Productivity Growth. Organization for Economic Co-operation and Development, Paris. doi: 10.1787/9789264194519-en.

Ray SC, Desli E (1997). "Productivity Growth, Technical Progress, and Efficiency Change in Industrialized Countries: Comment." The American Economic Review, 87(5), 1033-1039. ISSN 00028282. URL http://www.jstor.org/stable/2951340.

Shehata EAE, Mickaiel SKA (2015). "INDEX: Stata Module to Estimate Price, Quantity, and Value Index Numbers." Statistical Software Components No. S458005, Department of Economics, Boston College, Boston, Mass., USA, URL https://ideas.repec.org/c/ boc/bocode/s458005.html.

Simar L, Wilson PW (1998). "Sensitivity Analysis of Efficiency Scores: How to Bootstrap in Nonparametric Frontier Models." Management Science, 44(1), 49-61. URL https: //pubsonline.informs.org/doi/abs/10.1287/mnsc.44.1.49.

Simar L, Wilson PW (2002). "Non-Parametric Tests of Returns to Scale." European Journal of Operational Research, 139(1), 115 - 132. doi:10.1016/S0377-2217(01)00167-9.

StataCorp (2015). Stata Statistical Software: Release 14. StataCorp LP, College Station, TX. URL http://www.stata.com. 
The MathWorks, Inc (2017). MATLAB - The Language of Technical Computing, Version R2017b (9.3). Natick, Massachusetts. URL http://www.mathworks.com/products/ matlab/.

Zhu J (2014). Quantitative Models for Performance Evaluation and Benchmarking. Springer International Publishing AG, Cham, Switzerland. doi:10.1007/978-3-319-06647-9.

Zofío JL (2007). "Malmquist Productivity Index Decompositions: A Unifying Framework." Applied Economics, 39(18), 2371-2387. URL http://www.tandfonline.com/doi/abs/ $10.1080 / 00036840600606260$.

\author{
Affiliation: \\ José L. Zofío \\ Department of Economics \\ Universidad Autónoma de Madrid \\ 28049 Madrid, Spain \\ E-mail: jose.zofio@uam.es \\ URL: http://www.tfptoolbox.com/
}

